\title{
Metabolites from marine invertebrates and their symbiotic microorganisms: molecular diversity discovery, mining, and application
}

\author{
Lu Liu $^{1,2,3} \cdot$ Yao-Yao Zheng ${ }^{1,2} \cdot$ Chang-Lun Shao ${ }^{1,2} \cdot$ Chang-Yun Wang ${ }^{1,2,4}$
}

Received: 10 September 2019 / Accepted: 12 November 2019 / Published online: 4 December 2019

(c) The Author(s) 2019

\begin{abstract}
Metabolites from marine organisms have proven to be a rich source for the discovery of multiple potent bioactive molecules with diverse structures. In recent years, we initiated a program to investigate the diversity of the secondary metabolites from marine invertebrates and their symbiotic microorganisms collected from the South China Sea. In this review, representative cases are summarized focusing on molecular diversity, mining, and application of natural products from these marine organisms. To provide a comprehensive introduction to the field of marine natural products, we highlight typical molecules including their structures, chemical synthesis, bioactivities and mechanisms, structure-activity relationships as well as biogenesis. The mining of marine-derived microorganisms to produce novel secondary metabolites is also discussed through the OSMAC strategy and via partial chemical epigenetic modification. A broad prospectus has revealed a plethora of bioactive natural products with novel structures from marine organisms, especially from soft corals, gorgonians, sponges, and their symbiotic fungi and bacteria.
\end{abstract}

Keywords Marine invertebrates $\cdot$ Symbiotic microorganisms $\cdot$ Marine natural products $\cdot$ Molecular diversity · Bioactivities · Marine drugs

\section{Introduction}

Edited by Chengchao Chen.

Chang-Lun Shao

shaochanglun@ouc.edu.cn

$\triangle$ Chang-Yun Wang

changyun@ouc.edu.cn

1 Key Laboratory of Marine Drugs, the Ministry of Education of China, School of Medicine and Pharmacy, Ocean University of China, Qingdao 266003, China

2 Laboratory for Marine Drugs and Bioproducts, Qingdao National Laboratory for Marine Science and Technology, Qingdao 266237, China

3 Single-Cell Center, CAS Key Laboratory of Biofuels and Shandong Key Laboratory of Energy Genetics, Qingdao Institute of Bioenergy and Bioprocess Technology, Chinese Academy of Sciences, Qingdao 266101, China

4 Institute of Evolution and Marine Biodiversity, Ocean University of China, Qingdao 266003, China
The marine environment has been proven to be a rich source of new bioactive natural products for drug discovery. Coral reefs are among the most productive ecosystems and are a source of a large group of structurally unique biosynthetic products. To date, more than 40,000 marine natural products (MNPs) have been identified from various marine organisms, such as sponges, cnidarians, tunicates, molluscs, echinoderms, bryozoans, red algae, brown algae, green algae, and microorganisms (Carroll et al. 2019; Deshmukh et al. 2017; Jiménez 2018; Leal et al. 2016; Newman and Cragg 2016b). The upward trend in the discovery of new MNPs sourced from marine microorganisms continues unabated and now represents 57\% of the total new MNPs reported in 2017 (Carroll et al. 2019). Based upon the putative biogenetic origins, these MNPs can be classified as polyketides, terpenoids, alkaloids, steroids, lactones, peptides, phenols, and lipids. Also, a large proportion of MNPs display interesting pharmaceutical activities, such as cytotoxic, antimicrobial, hypolipidemic, anti-inflammatory, antimalarial, analgesic, and antiasthmatic activities (According to the following 
websites: http://marinepharmacology.midwestern.edu; Jiménez 2018). Hence, MNPs are considered as an excellent and potentially valuable source for new chemical entities with novel structures and distinct mechanisms of action. To date, there have been 13 approved therapeutic agents that could be considered derivatives of MNPs (Altmann 2017; Jiménez 2018; Newman 2019; Pereira et al. 2019). Moreover, more than $30 \mathrm{MNP}$ derivatives constitute the global marine pharmaceutical clinical pipeline in Phases III, II or I of drug development (According to the following websites: http:// marinepharmacology.midwestern.edu; http://pharma.id.infor ma.com (accessed on August 6, 2019); Jiménez 2018; Newman 2019; Pereira et al. 2019). The significant potential for new drug development based on MNPs in all disease areas has been previously discussed (Newman and Cragg 2016a).

Marine invertebrates have proven to be a primary source of bioactive MNPs, as many serve as chemical defense tools against predators, competitors and other ecological pressures. It has been demonstrated that the true origin of most MNPs appears to be the microorganisms living in concert with invertebrates. Most invertebrates are sessile, soft-bodied and move slowly, and are thus subject to potential parasite predation and detrimental microbial colonization. Therefore, they require a complex arsenal of secondary metabolites produced by their symbiotic microorganisms to facilitate a form of chemical defense (Jiménez 2018; Wang et al. 2008). This is likely the reason why MNPs from marine invertebrates and their symbiotic microorganisms are a rich sources of diverse and bioactive secondary metabolites (Martins et al. 2014; Mayer et al.
2010; Newman and Cragg 2016b). The chemical ecology underlying invertebrate-microorganism interactions provides a great opportunity for natural product chemists to mine for novel drug discovery. Therefore, the invertebrates and the abundant microorganisms in their ecosystems have attracted widespread attention for producing novel structural metabolites with potential bioactive applications (Blunt et al. 2018).

In the recent decade, the China Sea, especially the South China Sea, has become a hot spot in searching for novel bioactive MNPs. The invertebrates including sponges, soft corals, gorgonians and tunicates are prolific in the coral reefs in the South China Sea, and the microorganisms associated with these invertebrates have been demonstrated as a distinctive source for new bioactive MNPs.

In recent years, we have initiated a research program to find biological active MNPs based on marine chemical ecology (Figs. 1, 2) (Hou et al. 2015, 2019a; Wang et al. 2008). A total of 709 MNPs including 307 new compounds have been obtained from marine invertebrates and their symbiotic microorganisms collected from the South China Sea. In this review, we summarize the representative 287 MNPs (Table 1) obtained by our group, highlighting multiple structural types of compounds and demonstrating discovery, diversity, compound mining, and bioactive application. The goal is to provide further inspiration for the discovery of bioactive MNPs and subsequent drugs development.
Fig. 1 Collection positions for marine invertebrates and microorganisms in the South China Sea. The red points represent the sampling sites

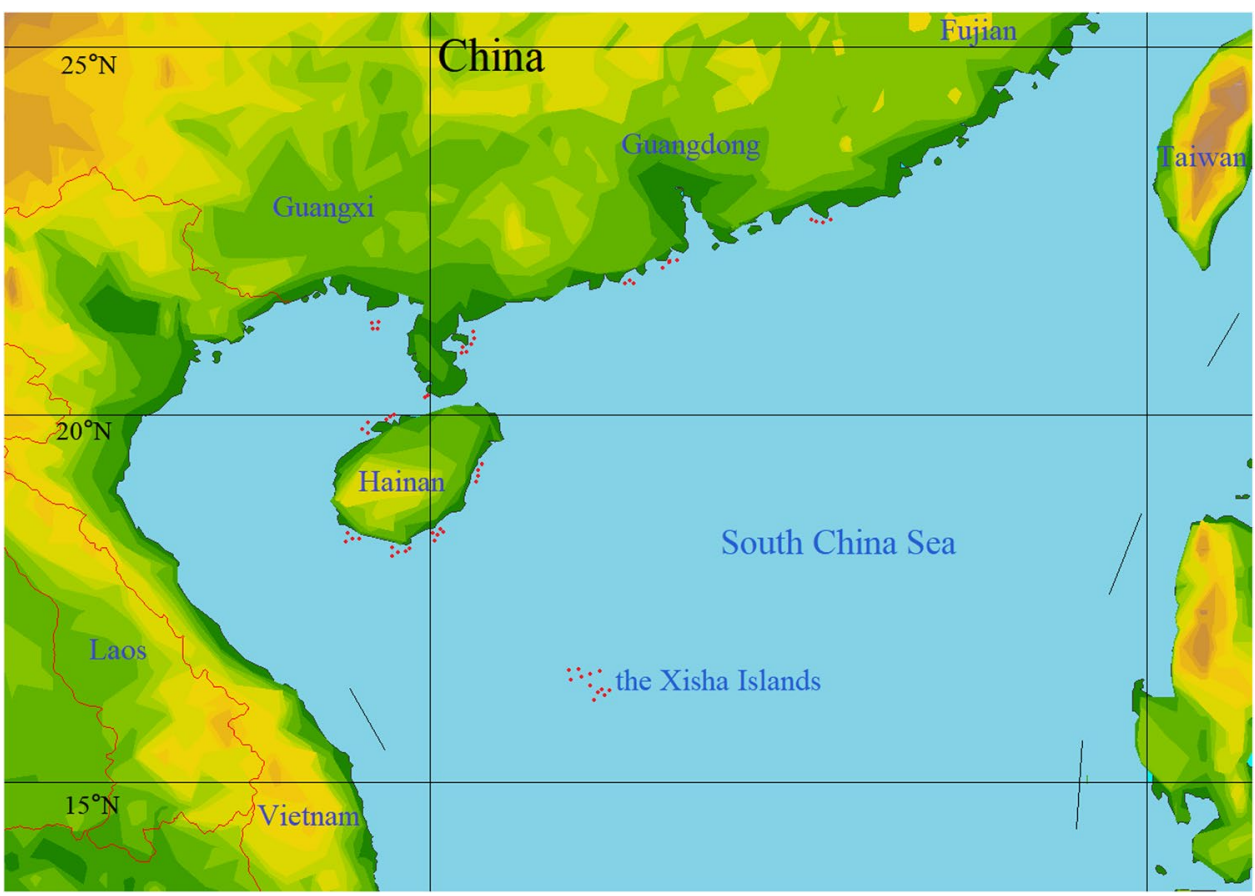


Fig. 2 The coral reef ecosystem and the chemical defensive metabolites with chemoecological effects

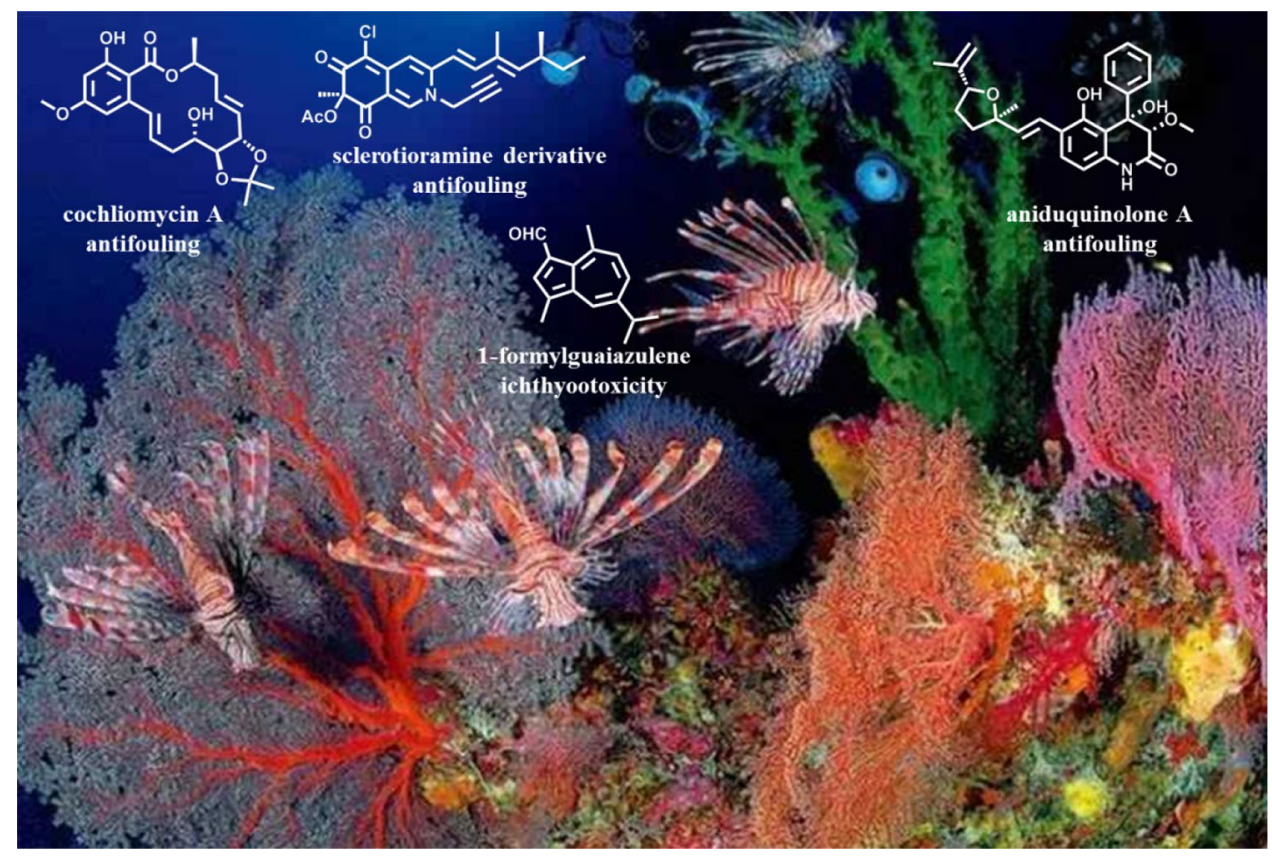

\section{Macrocyclic lactones}

Macrocyclic lactones derived from marine organisms have attracted much attention on account of their multiple potent biological activities including antitumor (Hirata and Uemura 1986; Isaka et al. 2002, 2009; Qi and Ma 2011; Suo et al. 2018), antifungal (Shier et al. 2001), and antimalarial activities (Isaka et al. 2009). For instance, caniferolides A and B, two glycosylated 36-membered polyol macrolides from marine-derived Streptomyces sp., showed pronounced antifungal activity (Perez-Victoria et al. 2019). Peloruside E, a macrolide from the New Zealand marine sponge Mycale hentscheli, displayed potent antiproliferative activity (Hong et al. 2018). As part of our current research, 88 macrolides with unique structures and significant biological properties have been obtained from marine invertebrates and their symbiotic microorganisms (Liu et al. 2014; Shao et al. 2011, 2015a, 2018). To obtain more valuable undiscovered natural compounds, multiple strategies and methods, including OSMAC and chemical epigenetic manipulation, were applied to microorganisms. Structural modification and chemical synthesis were performed to provide more derivatives, and the structure-activity relationships were discussed (Zhang et al. 2017). The molecular mechanisms of compounds with strong activities were also investigated (Wang et al. 2016).

Three new 14-membered resorcylic acid lactones (RALs), including compounds $\mathbf{1}$ and $\mathbf{2}$ (cochliomycins A and B) with a rare natural acetonide group and $\mathbf{3}$ (cochliomycin $\mathrm{C}$ ) with a 5-chloro substituted lactone (Fig. 3), were isolated from the culture broth of Cochliobolus lunatus (M351) isolated from the gorgonian Dichotella gemmacea collected from the
South China Sea (Shao et al. 2011). In an antifouling assay, compound 1 (cochliomycin A) showed significant antifouling activity against the barnacle Amphibalanus (= Balanus) amphitrite at nontoxic concentrations $\left(\mathrm{EC}_{50}=1.2 \mu \mathrm{g} / \mathrm{mL}\right)$. Thus, compound $\mathbf{1}$ merits further investigation as a molecular model for the discovery of new antifouling molecules (Shao et al. 2011).

The molecular mechanism underlying antifouling activity of 1 against the cyprids of barnacle A. amphitrite has been investigated using the isobaric tags for the relative or absolute quantification (iTRAQ) labeling proteomic method (Wang et al. 2016). Differentially expressed proteins were examined by analyzing the changes in the proteome of $A$. amphitrite cyprids in response to $\mathbf{1}$ treatment. The results suggested that compound $\mathbf{1}$ affected the cytochrome P450, glutathione $S$-transferase (GST) and NO/cGMP pathways. The results of real-time PCR further demonstrated that the NO/cGMP pathway was activated in response to 1 . Larval settlement assays suggested that $S$-methylisothiourea sulfate (SMIS) and $1 H$-(1,2,4)oxadiazolo[4,3-a]quinoxalin-1-one (ODQ) rescued cyprids from 1 (cochliomycin A)-induced inhibition of larval settlement. These findings supported the hypothesis that $\mathbf{1}$ inhibited barnacle larval settlement through stimulating the NO/cGMP pathway (Wang et al. 2016).

However, further research on compound $\mathbf{1}$ and its analogues was hindered by strain-specific variation of the strain C. lunatus (M351), especially altering metabolite profiles when re-cultured. Fortunately, another fungal strain $C$. lunatus (TA26-46) derived from the sea anemone Palythoa haddoni was also found to produce 14-membered RALs. Three new 14-membered RALs, 4-6 (cochliomycins D-F) 
Table 1 Representative compounds and their original organisms

\begin{tabular}{|c|c|c|c|c|}
\hline Compd. & Names & $\begin{array}{c}\text { Original } \\
\text { organisms }\end{array}$ & Species & Ref. \\
\hline \multicolumn{5}{|c|}{ Macrocyclic lactones } \\
\hline $1-3$ & Cochliomycins A-C & $\begin{array}{l}\text { Gorgonian-derive } \\
\text { d fungus }\end{array}$ & C. lunatus M351 & Shao et al. (2011) \\
\hline $4-6$ & Cochliomycins D-F & \multirow{9}{*}{$\begin{array}{l}\text { Sea } \\
\text { anemone-derived } \\
\text { fungus }\end{array}$} & \multirow[t]{9}{*}{ C. lunatus TA26-46 } & \multirow[t]{9}{*}{ Liu et al. (2014) } \\
\hline 7 & $\left(7^{\prime} E\right)-6 '-O x o z e a e n o l$ & & & \\
\hline 8 & Deoxy-aigialomycin C & & & \\
\hline 9 & LL-Z1640-2 & & & \\
\hline 9a & Acetonide derivative of 9 & & & \\
\hline 10 & Zeaenol & & & \\
\hline 11 & LL-Z1640-1 & & & \\
\hline 12 & Paecilomycin F & & & \\
\hline 13 & Aigialomycin B & & & \\
\hline 14-29 & Derivatives from $\mathbf{9}$ and $\mathbf{1 0}$ & \multicolumn{2}{|l|}{ Semisynthetic } & Zhang et al. (2017) \\
\hline 30 & 5-Bromozeaenol & \multirow{2}{*}{$\begin{array}{l}\text { Sea } \\
\text { anemone-derived } \\
\text { fungus }\end{array}$} & \multirow[t]{2}{*}{ C. lunatus TA26-46 } & \multirow[t]{2}{*}{ Zhang et al. (2014) } \\
\hline 31 & 3,5-Dibromozeaenol & & & \\
\hline 32 & Borrelidin & \multirow{2}{*}{$\begin{array}{l}\text { Sponge-derived } \\
\text { actinomycete; } \\
\text { gorgonian-derived } \\
\text { fungus }\end{array}$} & \multirow{2}{*}{$\begin{array}{l}\text { S. rochei } \mathrm{MB} 037 ; R \text {. } \\
\text { similis } 35\end{array}$} & \multirow[t]{2}{*}{ Yu et al. (2019) } \\
\hline 33-35 & Borrelidins F, J, K & & & \\
\hline 36 & Bastimolide A & \multirow[t]{4}{*}{ Cyanobacterium } & \multirow[t]{4}{*}{ O. hirsute } & \multirow[t]{2}{*}{ Shao et al. (2015a) } \\
\hline 36a & Nona-p-nitrobenzoate derivative of 32 & & & \\
\hline 37 & Bastimolide B & & & \multirow[t]{2}{*}{ Shao et al. (2018) } \\
\hline 38 & 2-(E)-Bastimolide A & & & \\
\hline \multicolumn{5}{|c|}{ Anthraquinones } \\
\hline 39 & 4a-epi-9 $\alpha$-Methoxydihydrodeoxybostrycin & \multirow{9}{*}{$\begin{array}{l}\text { Sea } \\
\text { anemone-derived } \\
\text { fungus }\end{array}$} & \multirow{9}{*}{$\begin{array}{l}\text { Nigrospora sp. } \\
\text { ZJ-2010006 }\end{array}$} & \multirow[t]{10}{*}{ Yang et al. (2012) } \\
\hline 40 & 10-Deoxybostrycin & & & \\
\hline 41 & Nigrosporin B & & & \\
\hline 42 & $9 \alpha$-Hydroxydihydrodesoxybostrycin & & & \\
\hline 43 & $9 \alpha$-Hydroxyhalorosellinia A & & & \\
\hline 44 & 4-Deoxybostrycin & & & \\
\hline 45 & Bostrycin & & & \\
\hline 46 & $\begin{array}{l}\text { 3,5,8-Trihydroxy-7-methoxy-2 } \\
\text {-methylanthracene-9,10-dione }\end{array}$ & & & \\
\hline 47 & Austrocortirubin & & & \\
\hline $\begin{array}{c}44 \mathrm{a}, \\
45 \mathrm{a}, \\
46 \mathrm{a}-46 \mathrm{~g} \\
, 47 \mathrm{a} \\
\end{array}$ & Acetyl derivatives of $\mathbf{4 4 - 4 7}$ & \multicolumn{2}{|l|}{ Semisynthetic } & \\
\hline $48-51$ & Tetrahydroaltersolanols C-F & \multirow{3}{*}{$\begin{array}{l}\text { Soft coral-derived } \\
\text { fungus }\end{array}$} & \multirow{3}{*}{$\begin{array}{l}\text { Alternaria } \mathrm{sp} . \\
\text { ZJ-2008003 }\end{array}$} & \multirow[t]{3}{*}{ Zheng et al. (2012) } \\
\hline 52 & Dihydroaltersolanol A & & & \\
\hline 53-57 & Alterporriol N-R & & & \\
\hline \multicolumn{5}{|c|}{ Azaphilones } \\
\hline $58-60$ & Pinophilins D-F & \multirow{6}{*}{$\begin{array}{l}\text { Gorgonian-derive } \\
\text { d fungus }\end{array}$} & \multirow{6}{*}{$\begin{array}{l}\text { P. pinophilum } \\
\text { XS-20090E18 }\end{array}$} & Zhao et al. (2015a) \\
\hline 61 & Sch 1385568 & & & \\
\hline 62 & Pinophilin B & & & \\
\hline 63 & Sch 725680 & & & \\
\hline 64 & (-)-Mitorubrin & & & \\
\hline 65 & (-)-Mitorubrinol & & & \\
\hline
\end{tabular}


Table 1 (continued)

\begin{tabular}{|c|c|c|c|c|}
\hline 66 & (-)-Mitorubrinic acid & & & \\
\hline $67-68$ & Penicilazaphilones D and E & \multirow{5}{*}{$\begin{array}{l}\text { Sponge-derived } \\
\text { fungus }\end{array}$} & \multirow[t]{5}{*}{ P. sclerotiorum } & \multirow[t]{5}{*}{ Wang et al. (2018) } \\
\hline 69 & $(+)$-Sclerotiorin & & & \\
\hline 70 & Geumsanol C & & & \\
\hline 71 & WB & & & \\
\hline 72 & Geumsanol G & & & \\
\hline 73-102 & Sclerotioramine derivatives of $\mathbf{6 9}$ & \multicolumn{2}{|l|}{ Semisynthetic } & Wei et al. (2017) \\
\hline \multicolumn{5}{|c|}{ Alkaloids } \\
\hline 103 & $( \pm)$-Pestaloxazine A & $\begin{array}{l}\text { Soft coral-derived } \\
\text { fungus }\end{array}$ & Pestalotiopsis sp. & Jia et al. (2015) \\
\hline 104 & Aniduquinolone A & \multirow{4}{*}{$\begin{array}{l}\text { Gorgonian } \\
\text { coral-derived } \\
\text { fungus }\end{array}$} & \multirow[t]{4}{*}{ Scopulariopsis sp. } & \multirow[t]{4}{*}{ Shao et al. (2015b) } \\
\hline $105-106$ & Aflaquinolones A and D & & & \\
\hline 107 & 6-Deoxyaflaquinolone E & & & \\
\hline 108-109 & Aflaquinolones $\mathrm{F}$ and $\mathrm{G}$ & & & \\
\hline 110 & 22-O-(N-Me- $L$-valyl)-Aflaquinolone $\mathrm{B}$ & \multirow{2}{*}{$\begin{array}{l}\text { Gorgonian-derive } \\
\text { d fungus }\end{array}$} & \multirow[t]{2}{*}{ Aspergillus sp. } & \multirow[t]{2}{*}{ Chen et al. (2014a) } \\
\hline 111 & 22-O-(N-Me- $L$-valyl)-21-epi-Aflaquinolone B & & & \\
\hline 112 & Di(1H-indol-3-yl)methane & \multirow[t]{8}{*}{ Bacterium } & \multirow{8}{*}{$\begin{array}{l}\text { P. denitrificans } \\
\text { UST4-50 }\end{array}$} & \multirow[t]{8}{*}{ Wang et al. (2015a) } \\
\hline 113 & Vibrindole A & & & \\
\hline 114 & 3,3'-Di-1H-indol-3-yl-1,2-propandiol & & & \\
\hline 115 & Tri(1H-indol-3-yl)methane & & & \\
\hline 116 & 1,2,2-Tri(1H-indol-3-yl) ethanone & & & \\
\hline 117 & Arsindoline A & & & \\
\hline 118 & 3, 3'-(Phenylmethylene) bis- $1 H$-indole & & & \\
\hline 119 & $\begin{array}{l}\text { (4-(Di(1H-indol-3-yl)methyl) phenol } \\
\text { (DIM-Ph-4-OH) }\end{array}$ & & & \\
\hline 120 & $\begin{array}{l}\text { 2-(Phenyl(2-phenyl-1H-indol-3-yl)methyl)malon } \\
\text { onitrile }\end{array}$ & \multirow{4}{*}{\multicolumn{2}{|c|}{ Synthetic }} & \multirow[t]{4}{*}{ Wen et al. (2015) } \\
\hline 121 & $\begin{array}{l}\text { 4-Hydroxy-3-(phenyl(2-phenyl-1H-indol-3-yl)me } \\
\text { thyl)- } 2 H \text {-chromen-2-one }\end{array}$ & & & \\
\hline 122 & 4-(Phenyl(2-phenyl-1H-indol-3-yl)methyl)phenol & & & \\
\hline 123 & $\begin{array}{l}\text { 2-Phenyl-3-(phenyl(2,4,6-trimethoxyphenyl)meth } \\
\text { yl)-1H-indole }\end{array}$ & & & \\
\hline \multicolumn{5}{|c|}{ Terpenoids } \\
\hline 124 & Aspergiterpenoid A & \multirow{4}{*}{$\begin{array}{l}\text { Sponge-derived } \\
\text { fungus }\end{array}$} & \multirow{4}{*}{$\begin{array}{l}\text { Aspergillus sp. } \\
\text { ZJ-2008004 }\end{array}$} & \multirow[t]{4}{*}{ Li et al. (2012a) } \\
\hline 125 & (-)-Sydonol & & & \\
\hline 126 & (-)-Sydonic acid & & & \\
\hline 127 & $\begin{array}{l}\text { (-)-5-(Hydroxymethyl)-2-(2',6',6'-trimethyltetrah } \\
\text { ydro-2H-pyran-2-yl)phenol }\end{array}$ & & & \\
\hline 128-138 & Carteriofenones A-K & Sponge & C. foliascens & Cao et al. (2015) \\
\hline 139-141 & Euplexaurenes A-C & \multirow[t]{2}{*}{ Gorgonian } & \multirow{2}{*}{$\begin{array}{l}\text { Euplexaura sp. } \\
\text { GXWZ-05 }\end{array}$} & \multirow[t]{2}{*}{ Cao et al. (2017) } \\
\hline 142 & Anthogorgiene P & & & \\
\hline 143-144 & Ochracenoids A and B & \multirow[t]{5}{*}{ Gorgonian } & A. ochracea & Zheng et al. (2014) \\
\hline 145 & 1-Formylguaiazulene & & & \\
\hline 146 & 1-formyl-4-methyl-7-isopropylazulene & & & \\
\hline 147 & Ketolactone & & & \\
\hline 148 & 3,8-Dimethyl-5-isopropyl-6-formylindenone & & & \\
\hline 149-154 & Lobophytumins A-F & Soft coral & S. compacta & Li et al. (2011) \\
\hline 155 & $(-)$-Germacrene D & & & \\
\hline 156 & Sarcophytol-A & Soft coral & S. infundibuliforme & Wang et al. (2011b) \\
\hline 157 & Sarcophytol-A acetate & & & \\
\hline 158 & Sarcophytol-H & & & \\
\hline 159 & Sarcophytonolid-J & & & \\
\hline
\end{tabular}


Table 1 (continued)

\begin{tabular}{|c|c|c|c|c|}
\hline 160 & Sarcophytol B & Soft coral & Sarcophyton sp. & Cao et al. (2013) \\
\hline 161 & Juncin P & \multirow[t]{2}{*}{ Gorgonian } & \multirow[t]{2}{*}{ D. fragilis } & \multirow[t]{2}{*}{ Zhou et al. (2011) } \\
\hline 162 & Junceellolide D & & & \\
\hline \multicolumn{5}{|l|}{ Steroids } \\
\hline $163-166$ & Echrebsteroids A-D & Gorgonian & E. rebekka & Cao et al. (2014) \\
\hline 167 & $15 \beta$-Hydroxypregna-1,4,20-trien-3-one & \multirow[t]{4}{*}{ Gorgonian } & \multirow[t]{4}{*}{ Carijoa sp. } & \multirow[t]{4}{*}{ Zhao et al. (2013) } \\
\hline 168 & $15 \beta$-Acetoxypregna1,4,20-trien-3-one & & & \\
\hline 169 & 18-Acetoxypregna-1,4,20-trien-3-one & & & \\
\hline 170 & Pregna-1,4,20-trien3-one & & & \\
\hline 171-182 & Subergorgiaols A-L & \multirow[t]{3}{*}{ Gorgonian } & \multirow[t]{3}{*}{ S. rubra } & \multirow[t]{3}{*}{ Sun et al. (2015) } \\
\hline 183 & Astrogorgiadiol & & & \\
\hline 184-186 & Calicoferols C, F, A & & & \\
\hline 187 & $(3 S, 23 R, 24 S)$-Ergost-5-ene-3 $\beta, 23 \alpha, 25$-triol & \multirow[t]{4}{*}{ Soft coral } & \multirow[t]{4}{*}{ Sinularia $\mathrm{sp}$. } & \multirow[t]{4}{*}{ Li et al. (2012b) } \\
\hline 188 & $(24 S)$-Ergostane-6-acetate- $3 \beta, 5 \alpha, 6 \beta, 25$-tetraol & & & \\
\hline 189 & $(24 S)$-Ergostane-6-acetate- $3 \beta, 6 \beta, 12 \beta, 25$-tetraol & & & \\
\hline 190 & $\begin{array}{l}\text { 24-Methylenecholestane-3 } \beta, 5 \alpha, 6 \beta \text {-triol-6-monoac } \\
\text { etate }\end{array}$ & & & \\
\hline 191-193 & Topsensterols A-C & Sponge & Topsentia sp. & Chen et al. (2016) \\
\hline 194 & $3 \alpha$-Hydroxy-7-ene-6,20-dione & \multirow{7}{*}{$\begin{array}{l}\text { Gorgonian-derive } \\
\text { d fungus }\end{array}$} & \multirow{7}{*}{$\begin{array}{l}\text { Cladosporium sp. } \\
\text { WZ-2008-0042 }\end{array}$} & \multirow[t]{7}{*}{ Yu et al. (2018) } \\
\hline 195 & $5 \alpha, 8 \alpha$-Epidioxy-ergosta- $6,9,22 E$-triene $3 \beta$-ol & & & \\
\hline 196 & $5 \alpha, 8 \alpha$-Epidioxy-ergosta- $6,22 E$-dien- $3 \beta$-ol & & & \\
\hline 197 & Ergosta-7,22E-diene- $3 \beta, 5 \alpha, 6 \beta$-triol & & & \\
\hline 198 & $3 \beta, 5 \alpha$-Dihydroxy- $6 \beta$-methoxyergosta7,22-diene & & & \\
\hline 199 & Ergosterol peroxide & & & \\
\hline 200 & Stigma-5-en-3- $O-\beta$-glucopyranoside & & & \\
\hline \multicolumn{5}{|c|}{ Phenylpropanoids } \\
\hline 201-203 & Penicimarins A-C & \multirow{5}{*}{$\begin{array}{l}\text { Sponge-derived } \\
\text { fungus }\end{array}$} & \multirow{5}{*}{$\begin{array}{l}\text { Penicillium sp. } \\
\text { MWZ14-4 }\end{array}$} & \multirow[t]{5}{*}{ Qi et al. (2013) } \\
\hline 204-205 & Aspergillumarins B and A & & & \\
\hline 206-208 & Penicimarins D-F & & & \\
\hline 209 & Sescandelin B & & & \\
\hline 210 & 5,6,8-Trihydroxy-4-(1'-hydroxyethyl)isocoumarin & & & \\
\hline 211-222 & Corynechromones A-L & $\begin{array}{l}\text { Sponge-derived } \\
\text { fungus }\end{array}$ & $\begin{array}{l}\text { C. cassiicola } \\
\text { XS-200900I7 }\end{array}$ & Zhao et al. (2015b) \\
\hline 223-234 & $( \pm)$-Eurotiumides A-G & $\begin{array}{l}\text { Gorgonian-derive } \\
\text { d fungus }\end{array}$ & $\begin{array}{l}\text { Eurotium } \mathrm{sp} . \\
\text { XS-200900E6 }\end{array}$ & Chen et al. (2014b) \\
\hline \multicolumn{5}{|l|}{ Peptides } \\
\hline 235-237 & Chrysogeamides A-C & \multirow{3}{*}{$\begin{array}{l}\text { Gorgonian } \\
\text { coral-derived } \\
\text { fungus }\end{array}$} & \multirow{3}{*}{$\begin{array}{l}\text { P. chrysogenum } \\
\text { TA01-16 }\end{array}$} & Hou et al. (2019b) \\
\hline 238-239 & Scopularides A and B & & & \\
\hline $240-244$ & Hrysogeamides D-H & & & \\
\hline $245-247$ & Asperversiamides A-C & $\begin{array}{l}\text { Coral-derived } \\
\text { fungus }\end{array}$ & $\begin{array}{l}\text { A. versicolor } \\
\text { CHNSCLM-0063 }\end{array}$ & Hou et al. (2019c) \\
\hline 248 & Penilumamide & Gorgonian-derive & Aspergillus sp. & Chen et al. (2014c) \\
\hline 249-251 & Penilumamides B-D & $\mathrm{d}$ fungus & XS-20090B15 & \\
\hline 252 & Asperpeptide A & & & \\
\hline Phenyl E & her Derivatives & & & \\
\hline 253 & Cordyol D & Gorgonian-derive & Aspergillus sp. & Chen et al. (2013b) \\
\hline 254 & 3,3'-O-Dimethyl-violaceol-I & $\mathrm{d}$ fungus & XS-20090066 & \\
\hline 255 & Cordyol C & & & \\
\hline 256 & 4-Methoxycarbonyl-diorcinol & & & \\
\hline 257 & Cordyol E & & & \\
\hline 258 & Diorcinol & & & \\
\hline $\begin{array}{c}256 a, \\
258 a-25\end{array}$ & Derivative of $\mathbf{2 5 6}$ and $\mathbf{2 5 8}$ & Semisynthetic & & \\
\hline
\end{tabular}


Table 1 (continued)

\begin{tabular}{|c|c|c|c|c|}
\hline $8 g$ & & & & \\
\hline 259-261 & Phomaethers A-C & \multirow{2}{*}{$\begin{array}{l}\text { Gorgonian-derive } \\
\text { d fungus }\end{array}$} & \multirow[t]{2}{*}{ Phoma sp. TA07-1 } & \multirow[t]{2}{*}{ Shi et al. (2017) } \\
\hline 262 & $\begin{array}{l}\text { 2,3'-Dihydroxy-4-methoxy-5',6-dimethyl } \\
\text { diphenyl ether }\end{array}$ & & & \\
\hline 263 & Talaromycin C & \multirow{5}{*}{$\begin{array}{l}\text { Gorgonian-derive } \\
\text { d fungus }\end{array}$} & \multirow[t]{2}{*}{ Talaromyces sp. } & \multirow[t]{2}{*}{ Chen et al. (2015) } \\
\hline 264 & Purpactin $\mathrm{C}^{\prime}$ & & & \\
\hline 265 & Pencillide & & \multirow{2}{*}{$\begin{array}{l}\text { P. pinophilum } \\
\mathrm{XS}-20090 \mathrm{E} 18\end{array}$} & \multirow[t]{2}{*}{ Zhao et al. (2015a) } \\
\hline 266 & Purpactin A & & & \\
\hline 267 & Tenellic acid A methyl ester & & Talaromyces sp. & Chen et al. (2015) \\
\hline
\end{tabular}

together with eight known analogues 1 (cochliomycin A), 7 ((7'E)-6'-oxozeaenol), 8 (deoxy-aigialomycin C), 9 (LLZ1640-2), 10 (zeaenol), 11 (LL-Z1640-1), 12 (paecilomycin F) and 13 (aigialomycin B) (Fig. 3) were obtained (Liu et al. 2014). Compounds 4, 6-9, and the acetonide derivative 9a displayed strong anti-larval settlement activity against $B$. amphitrite $\left(\mathrm{EC}_{50}=1.82\right.$ to $\left.22.5 \mu \mathrm{g} / \mathrm{mL} ; \mathrm{LC}_{50} / \mathrm{EC}_{50}>50\right)$, suggesting that they might be promising environmentally benign antifouling agents (Liu et al. 2014).

Noticeably, besides significant antifouling activity, compound 9 (LL-Z1640-2) produced by $C$. lunatus (TA26-46) exhibited promising inhibitory activity against phytopathogenic fungal strain Pestalotia calabae, with a minimum inhibitory concentration (MIC) value of $0.39 \mu \mathrm{mol} / \mathrm{L}$, 25 -fold stronger than that of the positive control, ketoconazole ( $\mathrm{MIC}=10 \mu \mathrm{mol} / \mathrm{L})$ (Liu et al. 2014). A fungicide whole plant assay suggested that $\mathbf{9}$ exhibited pronounced activity on the phytopathogenic fungus Plasmopara viticola preventative test at $6 \times 10^{-6}$ and concentration-dependent activity on the phytopathogenic fungus Phytophthora infestans preventative application at 200,60 , and $20 \times 10^{-6}$. Compound 9 could be considered as a promising new natural fungicide lead (Liu et al. 2014).

Further optimization of fermentation conditions of fungus $C$. lunatus (TA26-46) led to the isolation of two major natural products 9 (LL-Z1640-2) and 10 (zeaenol) (Fig. 3) with multi-gram quantities (Zhang et al. 2017). By one or two steps, we semi-synthesized six trace natural compounds 1 (cochliomycin A), 3 (cochliomycin C) (Fig. 3), 4 (cochliomycin D), 5 (cochliomycin E), 6 (cochliomycin F) and 7 ((7'E)-6'-oxozeaenol) (Fig. 3), and a series of derivatives 14-29 (Fig. 4) from compounds 9 and 10 with high yields (65-95\%) (Zhang et al. 2017). Compounds 14-16 displayed potent antiplasmodial activity against $P$. falciparum $\left(\mathrm{IC}_{50}=1.84,8.36\right.$, and $6.95 \mu \mathrm{mol} / \mathrm{L}$, respectively). The acetoxy groups and acetonide functionality might contribute to the antiplasmodial activity (Fig. 5). However, the chlorine atom has a negative effect on the activity. Very importantly, 14 and 15 were non-toxic with very safety and high therapeutic indices $\left(\mathrm{CC}_{50} / \mathrm{IC}_{50}>180\right)$, and thus represent potential promising leads for antiplasmodial drug discovery. In addition, 2-OH was found to be an important functionality for reducing toxicity. Only $\mathbf{1 4}$ showed antileishmanial activity against Leishmania donovani $\left(\mathrm{IC}_{50}=9.22 \mu \mathrm{mol} / \mathrm{L}\right)$. Most of the enone RALs showed high antileishmanial efficacy but poor therapeutic indices, suggesting that the $c i s$ or trans-functionality has a contribution to toxicity, and their antileishmanial activities may well be related to their toxicity (Fig. 5). Furthermore, $\mathbf{1 4}$ and $\mathbf{1 5}$ also displayed antiplasmodial activity against the neglected Chagas' disease causing Trypanosoma cruzi $\left(\mathrm{IC}_{50}=11.9\right.$ and $17.2 \mu \mathrm{mol} / \mathrm{L}$, respectively) (Zhang et al. 2017).

It should be noted that many gene clusters in marinederived fungi for the production of undiscovered secondary metabolites generally remain unexpressed under common laboratory culture conditions. In order to obtain uncharacterized metabolites, the chemical epigenetic perturbation method was applied to $C$. lunatus (TA26-46) to manipulate the silent fungal genes. Two new 14-membered RALs characterized with bromine substitution, 30 (5-bromozeaenol) and 31 (3,5-dibromozeaenol) (Fig. 6), were obtained from the culture treated with histone deacetylase inhibitor, sodium butyrate (Zhang et al. 2014). Compounds 30 and 31 represent the first examples of naturally occurring brominated RALs.

Facing the challenges of discovery repetition and silent biosynthetic pathways, only limited mining approaches were applied in our work to date. As well known, microorganisms are easily manipulated on the genetic level. With gene sequencing technology blooming, genetic techniques and bioinformatics algorithms will continue to provide more opportunities for disclosing the novel pathways within marine microorganisms to then enable exploration of the untapped valuable bioactive molecules encoded within.

Furthermore, co-culturing as an effective method to induce production of novel secondary metabolites from two interacting microbial strains was applied to mine macrocyclic lactones. In single strain cultivation, the sponge-derived actinomycete Streptomyces rochei MB037 could produce two 18-membered macrolides, 32 (borrelidin) and 33 (borrelidin F) (Yu et al. 2019). Interestingly, in the co-culture of 
<smiles>C/C=C/C[C@@H](O)[C@H]1OC(C)(C)O[C@@H]1/C=C\CC(C)OC(=O)c1c(O)cc(OC)cc1O</smiles>

cochliomycin A (1)<smiles>C/C=C/C[C@@H](O)[C@H](O)C(=O)/C=C/CC(C)OC(=O)c1c(O)cc(OC)cc1O</smiles>

cochliomycin E (5)<smiles>C/C=C\C=C/C[C@H](O)[C@H](O)C(=O)/C=C\CC(C)OC(=O)c1c(O)cc(OC)cc1O</smiles>

LL-Z1640-2 (9)

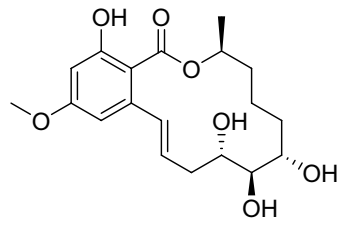

paecilomycin $\mathrm{F}$ (12)<smiles>C/C=C/C[C@H]1OC(C)(C)O[C@@H]1[C@@H](O)/C=C/CC(C)OC(=O)c1c(O)cc(OC)cc1O</smiles>

cochliomycin B (2)<smiles>C/C=C/CC[C@@H](O)C(O)C(=O)/C=C/CC(C)OC(=O)c1c(O)cc(OC)cc1O</smiles>

cochliomycin F (6)<smiles>COc1cc(O)c(C(=O)OC(C)C/C=C\C(=O)C2OC(C)(C)OC2C/C=C/c2cc(OC)cc(O)c2C)c(O)c1</smiles>

9a<smiles>COc1cc(O)c(C(=O)OC(C)C/C=C\C(O)[C@@H](O)[C@@H](O)C[C@@H]2CO2)c(O)c1</smiles>

aigialomycin $B$ (13)<smiles>COc1cc(O)c(C(=O)OC(C)CCC[C@H](O)[C@H](O)[C@H](O)C/C=C/c2cc(OC)c(OC)cc2Cl)c(O)c1</smiles>

cochliomycin C (3)<smiles>C/C=C/C[C@@H](O)C(O)C(=O)/C=C/CC(C)OC(=O)c1c(O)cc(OC)cc1O</smiles>

(7' E)-6'- oxozeaenol (7)<smiles>CC/C=C\C[C@H](O)C(O)C/C=C\CC(C)OC(=O)c1c(O)cc(OC)cc1O</smiles>

zeaenol (10)<smiles>C/C=C/C[C@@H](O)C(O)C(=O)/C=C\CC(C)OC(=O)c1c(O)cc(OC)cc1O</smiles>

cochliomycin D (4)<smiles>C/C=C/CC[C@H](O)[C@@H](O)C/C=C/CC(C)OC(=O)c1c(O)cc(OC)cc1O</smiles>

deoxy-aigialomycin C (8)<smiles>CCC(O)C(O)C(=O)CCCC(C)OC(=O)c1c(O)cc(OC)cc1/C=C\CC(C)O</smiles>

LL-Z1640-1 (11)

Fig. 3 14-Membered resorcylic acid lactones from the gorgonian-derived fungus $C$. lunatus (M351) (1-3) and the sea anemone-derived fungus C. lunatus (TA26-46) (4-13)

S. rochei MB037 and the gorgonian-derived fungus Rhinocladiella similis 35 , besides macrolides $\mathbf{3 2}$ and $\mathbf{3 3}, S$. roche $i$ MB037 was induced to produce two new fatty acids with a rare nitrile group, $\mathbf{3 4}$ (borrelidin J) and $\mathbf{3 5}$ (borrelidin K) (Fig. 7) (Yu et al. 2019), which are structurally related to borrelidins. Compounds $\mathbf{3 4}$ and $\mathbf{3 5}$ were derived from the actinomycete $S$. rochei MB037, suggesting that the silent hydrolytic enzyme genes for hydrolyzing lactone in the borrelidin macrolides could be activated by the co-culture approach. Notably, both $\mathbf{3 4}$ and 35, obtained only in coculture, exhibited stronger antibacterial activities against methicillin-resistant $S$. aureus than $\mathbf{3 2}$ and $\mathbf{3 3}$.

In addition to marine invertebrates and their symbiotic microorganisms, macrolides were also obtained from marine-derived bacteria. Compound 36 (bastimolide A) (Fig. 8), a polyhydroxy macrolide with a 40-membered ring, was obtained from a new genus of the tropical marine cyanobacterium Okeania hirsute (Shao et al. 2015a). Its complete structure and absolute configuration were unambiguously identified by X-ray diffraction analysis of the nona- $p$-nitrobenzoate derivative 36a (Fig. 8). Compound 36 is a complex 40-membered macrolactone with repeating 1,5-diol and 1,3-diol groups as well as a $t$-butyl group moiety which is quite rare among natural products. Compound $\mathbf{3 6}$ showed strong antimalarial activity against four resistant strains of P. falciparum $\left(\mathrm{IC}_{50}=80\right.$ to $\left.270 \mathrm{nmol} / \mathrm{L}\right)$, including TM90$\mathrm{C} 2 \mathrm{~A}$ (chloroquine, mefloquine, and pyrimethamine resistant), TM90-C2B (chloroquine, mefloquine, pyrimethamine, and atovaquone resistant), W2 (chloroquine and pyrimethamine resistant), and TM91-C235 (chloroquine, mefloquine, and pyrimethamine resistant). Although this compound displayed toxicity to the host Vero cells $\left(\mathrm{IC}_{50}=2.1 \mu \mathrm{mol} / \mathrm{L}\right)$, it still represents a potentially promising lead for antimalarial drug discovery (Shao et al. 2015a).

After discovery of the potent antimalarial 40-membered macrolide, continued investigation of the biologicallyactive and structurally-complex polyketides from $O$. hirsuta led to the isolation of a novel analogue 37 (bastimolide 
<smiles>COC(=O)O[C@H](C)C/C=C\[C@H]1OC(C)(C)OC1C/C=C/c1cc(OC)cc(OC)c1C(=O)O[Na]</smiles>

14

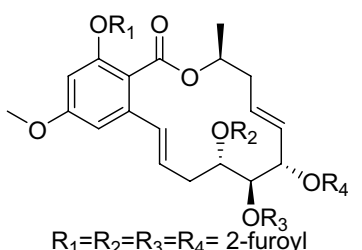

18

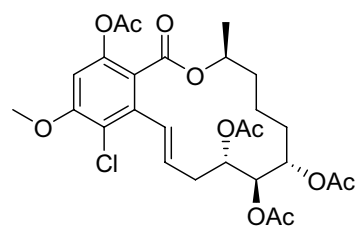

22<smiles></smiles><smiles>COc1cc(O)c(C(=O)OC(C)C/C=C\C=C/CC2OC(=O)C(OC(C)=O)C2OC(C)=O)c(O)c1</smiles>

15

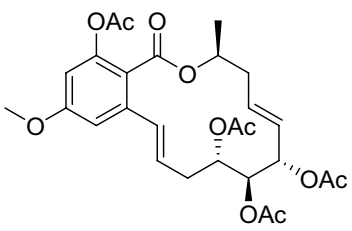

16

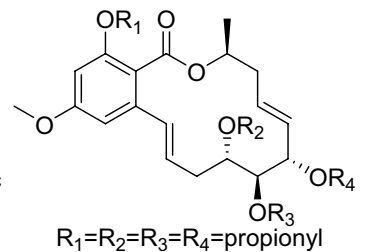

17<smiles>COc1cc(O)c(C(=O)OC(C)C/C=C\[C@H](O)[C@@H](O)[C@H](O)C/C=C/c2cc(Cl)c(OC)cc2Cl)c(O)c1</smiles>

19<smiles>COc1cc(O)c(C(=O)OC(C)C/C=C/CC(=O)O)c(/C=C/C[C@H]2OC(C)([13CH3])O[C@@H]2C)c1</smiles>

23<smiles>COc1cc(/C=C/CC2OCC/C=C\C(=O)C2OC(C)=O)c(C(=O)OC(C)C)c(OC(C)=O)c1</smiles>

27<smiles>C/C=C/C[C@@H](O)[C@H]1OC(C)(C)O[C@@H]1/C=C\CC(C)OC(=O)c1c(O)cc(OC)c(Cl)c1Cl</smiles>

20

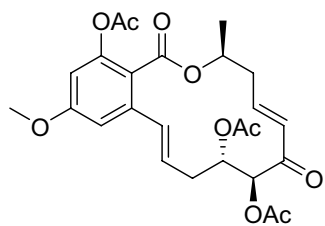

24<smiles>COc1cc(O)c(C(=O)OC(C)CCC[C@@H]2OC(C)(C)O[C@@H]2[C@H](O)C/C=C/c2c(Cl)c(OC)cc(OC)c2Cl)c(O)c1</smiles>

21

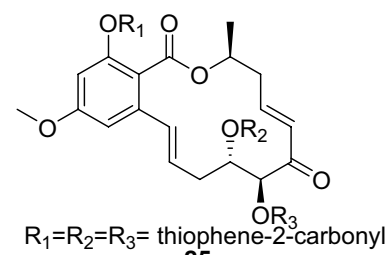

25

Fig. 4 Semisynthesized resorcylic acid lactone derivatives

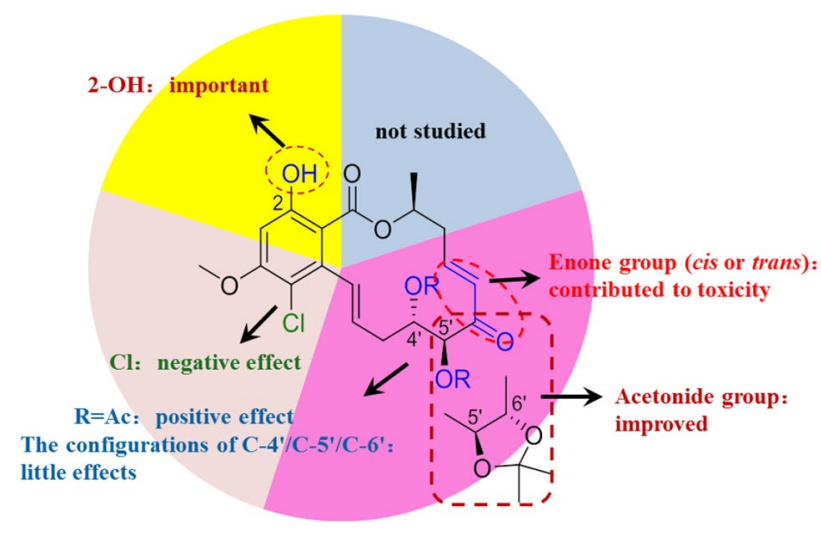<smiles>COc1cc(O)c(C(=O)OC(C)C/C=C/[C@@H](O)[C@@H](O)C(O)C/C=C/c2cc(Br)c(OC)cc2Br)c(O)c1</smiles>

5-bromozeaenol (30)<smiles>COc1c(Br)c(O)c(/C=C/C[C@H](O)[C@H](O)[C@H](O)/C=C/C[C@@H](C)O)c(C(=O)O)c1Br</smiles>

3,5-dibromozeaenol (31)

Fig. 6 Brominated resorcylic acid lactones from the sea anemonederived fungus $C$. lunatus (TA26-46) by chemical epigenetic manipulation

bond, 38 (2-(E)-bastimolide A) (Fig. 9), was also obtained. The natural product 37 showed pronounced antimalarial activity against chloroquine-sensitive $P$. falciparum strain HB3 $\left(\mathrm{EC}_{50}=5.72 \pm 0.65 \mu \mathrm{mol} / \mathrm{L}\right)$. Compound 38 showed even stronger antimalarial potency with an $\mathrm{EC}_{50}$ value of $1.41 \pm 0.47 \mu \mathrm{mol} / \mathrm{L}$. Thus, the bastimolides show considerable antimalarial activity and represent an intriguing new class of antimalarial agents (Shao et al. 2018). 


\section{Anthraquinones}

The diverse activities of anthraquinones isolated from marine-derived fungi with cytotoxic (Chen et al. 2013a; Xie et al. 2010; Zhu et al. 2012), antiviral (Huang et al. 2017), antioxidant (Li et al. 2016) and other activities have attracted many interests for drug discovery. For example, SZ-685C, a hydroanthraquinone isolated from the marine-derived $\mathrm{Hal}$ orosellinia fungus, exhibited potent cytotoxic activity (Chen et al. 2013a; Xie et al. 2010; Zhu et al. 2012). In our investigation of bioactive anthraquinone derivatives from marinederived fungi (Yang et al. 2012; Zheng et al. 2012), a total of 54 anthraquinone monomers and dimers have been isolated from Nigrospora sp., Alternaria sp. and other fungal genera. Analysis of anthraquinones with various chemical structures possessing interesting biological activities revealed the key structural features required for their activities.

Nine anthraquinone derivatives were obtained from a sea anemone-derived fungus Nigrospora sp. ZJ-2010006, including two new hydroanthraquinone analogues, 39 (4a-epi-9 $\alpha$-methoxydihydrodeoxybostrycin) and $\mathbf{4 0}$ (10-deoxybostrycin), together with seven known anthraquinone derivatives $\mathbf{4 1}$ (nigrosporin B), $\mathbf{4 2}$ (9 $\alpha$-hydroxydihy drodesoxybostrycin), $\mathbf{4 3}$ ( $9 \alpha$-hydroxyhalorosellinia A), $\mathbf{4 4}$ (4-deoxybostrycin), 45 (bostrycin), $\mathbf{4 6}$ (3,5,8-trihydroxy7-methoxy-2-methylanthracene-9,10-dione), 47 (austrocortirubin) (Fig. 10). Ten acetyl derivatives (44a, 45a, 46a-46g, 47a) (Fig. 10) were prepared by structural modification (Yang et al. 2012). Compound 39 represents the first case of a hydroanthraquinone with a 9-OMe group. Notably, $\mathbf{4 0}$ displayed significant antibacterial activity against Bacillus subtilis $(\mathrm{MIC}=625 \mathrm{nmol} / \mathrm{L})$. Compound 41 exhibited promising antibacterial activity against $B$. subtilis and $B$. cereus $(\mathrm{MICs}=312 \mathrm{nmol} / \mathrm{L})$. Additionally, the derivative 44a showed pronounced inhibitory activity against $B$. cereus $(\mathrm{MIC}=48.8 \mathrm{nmol} / \mathrm{L}), 25$-fold stronger than that of ciprofloxacin $(\mathrm{MIC}=1250 \mathrm{nmol} / \mathrm{L})$. These results demonstrated that hydroanthraquinone derivatives might be a promising source for antimicrobial agent discovery.

Structure-activity relationship analysis revealed that the introduction of the 4-OH/9-OH/10-OH groups had little effect on antibacterial activity (Fig. 11). Interestingly, transforming from a hydroxy group at $\mathrm{C}-3$ to an acetoxy

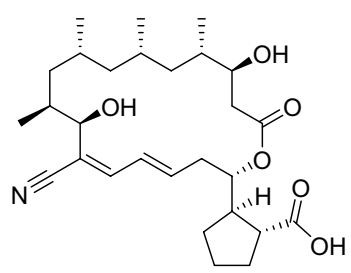

borrelidin (32)

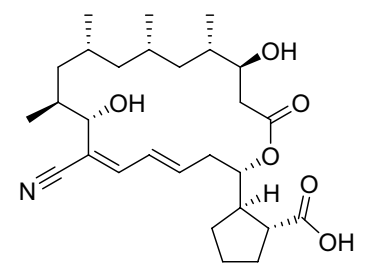

borrelidin $\mathrm{F}$ (33)

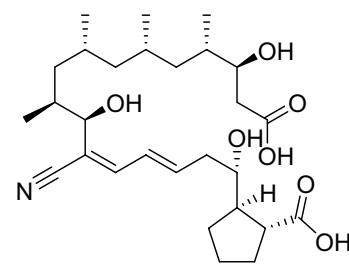

borrelidin $\mathrm{J}$ (34)

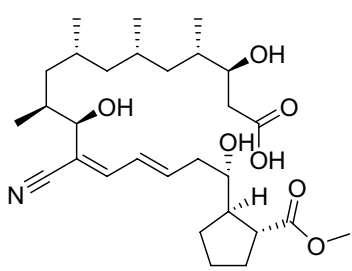

borrelidin K (35)

Fig. 7 18-Membered macrolides and structurally related fatty acids from the co-culture of the sponge-derived actinomycete $S$. rochei MB037 and the gorgonian-derived fungus Rhinocladiella similis 35

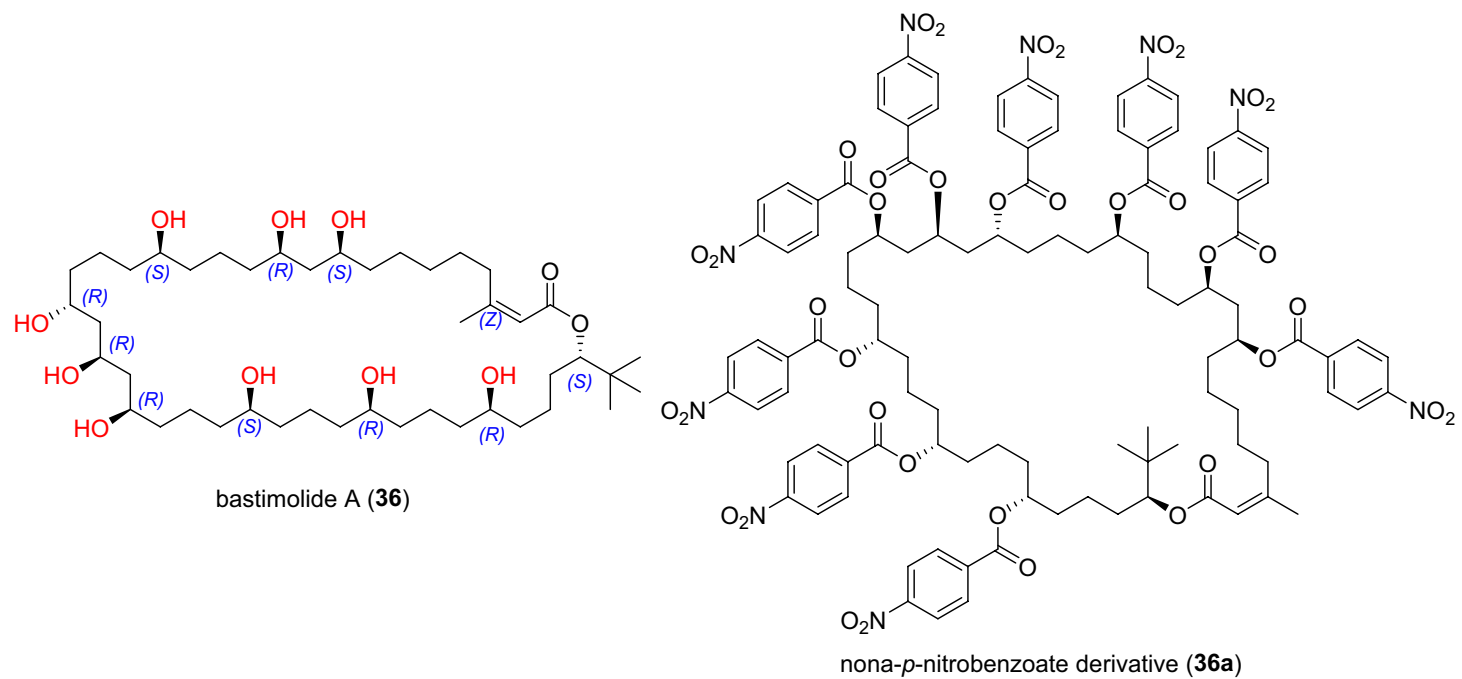

Fig. 8 Polyhydroxy macrolides from the cyanobacterium $O$. hirsute 


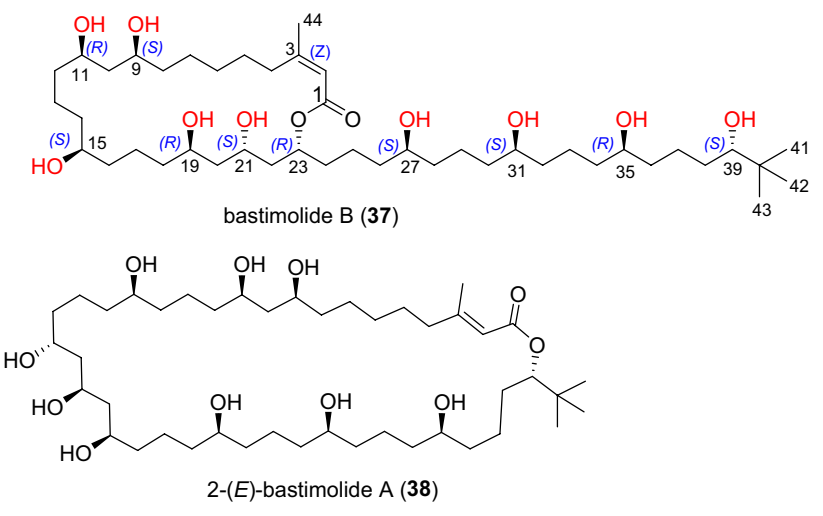

Fig. 9 Bastimolide B and isomerization product of bastimolide A from the cyanobacterium $O$. hirsute

group significantly increased selective antibacterial activity. The cycloaliphatic ring has a positive contribution to the antibacterial activity. Additionally, an aromatic B ring may be necessary for the antibacterial activity (Yang et al. 2012).

Ten anthraquinone derivatives were isolated from the culture of Sarcophyton soft coral-derived fungus Alternaria sp. ZJ-2008003 collected from the South China Sea, including five new hydroanthraquinone derivatives, 48-51 (tetrahydroaltersolanols C-F) and $\mathbf{5 2}$ (dihydroaltersolanol A), and five new alterporriol-type anthranoid dimers, 53-57 (alterporriols N-R) (Fig. 12) (Zheng et al. 2012). Compound 54 (alterporriol $\mathrm{O}$ ) was the first isolated alterporriol dimer with a C-4-C-4' linkage. Compound 48 (tetrahydroaltersolanol C) and $\mathbf{5 6}$ (alterporriol Q) showed antiviral activity against the Porcine Reproductive and Respiratory Syndrome Virus (PRRSV) $\left(\mathrm{IC}_{50}=65\right.$ and $22 \mu \mathrm{mol} / \mathrm{L}$, respectively). Compound $\mathbf{5 5}$ (alterporriol $\mathrm{P}$ ) exhibited cytotoxic activity against the PC-3 and HCT-116 cell lines $\left(\mathrm{IC}_{50}=6.4\right.$ and $8.6 \mu \mathrm{mol} / \mathrm{L}$, respectively) (Zheng et al. 2012).

\section{Azaphilones}

The azaphilone molecules are a structurally variable family of fungal polyketide metabolites with a highly oxygenated pyranoquinone bicyclic core and exhibiting multiple bioactivities such as cytotoxic (Yamada et al. 2008), antimicrobial (Che et al. 2002), antiviral (Wang et al. 2011a), and anti-inflammatory (Yasukawa et al. 2008) activities. For instance, sclerketide $\mathrm{C}$, an azaphilone analogous isolated from gorgonian-derived fungus Penicillium sclerotiorin CHNSCLM-0013, exhibited significant anti-inflammatory activity (Liu et al. 2019). Pleosporalone B from the culture of marine-derived fungus Pleosporales sp. CF09-1 and penicilazaphilone $\mathrm{C}$ from Penicillium sclerotiorum M-22 displayed potent antimicrobial activities (Cao et al. 2019; Zhou et al. 2016). Azaphilones have attracted much attention due to their fascinating structural features and distinguished bioactivities (Wei et al. 2017). Our previous work on metabolites produced by symbiotic microorganisms of marine invertebrates found 48 azaphilones, including 37 new compounds, many of which were reported to exhibit antifouling and antiviral activities (Wang et al. 2018; Wei et al. 2017; Zhao et al. 2015a). Desiring promising antifouling molecules, a one-step semisynthetic method was applied to discover more new azaphilonoids derivatives.

A series of azaphilone derivatives were isolated from the gorgonian-derived fungal strain, Penicillium pinophilum XS-20090E18, collected from the Xisha Islands coral reef, including three new azaphilone derivatives 58-60 (pinophilins D-F), together with six known azaphilone derivatives 61 (Sch 1385568), 62 (pinophilin B), 63 (Sch 725680), 64 ((-)-mitorubrin), 65 ((-)-mitorubrinol) and $66((-)$-mitorubrinic acid) (Fig. 13) (Zhao et al. 2015a). However, none of the obtained azaphilone derivatives showed antifouling, cytotoxic, or topoisomerase I (Topo I) inhibitory activity (Zhao et al. 2015a).

Six azaphilone derivatives were isolated from the sponge-derived fungus Penicillium sclerotiorum, including two new azaphilones 67 and 68 (penicilazaphilones $\mathrm{D}$ and $\mathrm{E})$, together with four known analogs, 69 ((+)-sclerotiorin), 70 (geumsanol C), 71 (WB (CAS No. 1701443-52-8, 6H-2-benzopyran-6-one,5-chloro3 -[(1E,3R,4R,5S)-3,4-dihydroxy-3,5-dimethyl-1-hepten-1-yl]-1,7,8,8a-tetrahydro-7,8-dihydroxy-7-methyl-,($7 R, 8 R, 8 \mathrm{a} S$ )) and 72 (geumsanol G) (Fig. 14) (Wang et al. 2018). Compound $\mathbf{6 7}$ was the second reported azaphilone with a $\mathrm{C} 4$ aliphatic side chain, and $\mathbf{6 8}$ was the first azaphilone with a tetrahydrofuran ring at C-3. Compound 69 demonstrated antiviral activity against $\mathrm{HSV}$ $\left(\mathrm{IC}_{50}=19.5 \mu \mathrm{mol} / \mathrm{L}\right)$ and EV71 $\left(\mathrm{IC}_{50}=132 \mu \mathrm{mol} / \mathrm{L}\right), 16$ and 3 -fold stronger than the positive control ribavirin, respectively (Wang et al. 2018).

On account of the significant bioactivities of $\mathbf{6 9}$ ((+)-sclerotiorin), 30 sclerotioramine derivatives 73-102 has been semi-synthesized from 69 by a one-step reaction (Fig. 15) (Wei et al. 2017). Most of them except 77, $\mathbf{7 8}, \mathbf{7 9}, 83$, and 99 showed strong anti-barnacle settlement activity against $B$. amphitrite. The aromatic amino-derivatives 84-88, 90-92, 94, 96-98, and 100-102 demonstrated potent antifouling activity; while only two aliphatic amino-derivatives $\mathbf{7 6}$ and $\mathbf{1 0 1}$ displayed antifouling activity. It should be noted that $\mathbf{7 6}$ and $\mathbf{1 0 1}$ showed promising activity $\left(\mathrm{EC}_{50}=0.94\right.$ and $0.47 \mu \mathrm{g} / \mathrm{mL}$, respectively) stronger than that of the commercial biocide Sea-Nine $211^{\mathrm{TM}}\left(\mathrm{EC}_{50}=1.2 \mu \mathrm{g} / \mathrm{mL}\right)$. Meanwhile, compounds 76 and 101 possess high therapeutic ratios $\left(\mathrm{LC}_{50} / \mathrm{EC}_{50} 53.2\right.$ and 106.4 , respectively), indicating that they may be antifouling candidates with low-toxicity for the development of new environmentally benign antifoulants (Wei et al. 2017). 


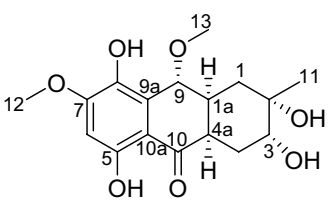

4a-epi-9a- methoxydihydrodeoxybostrycin (39)

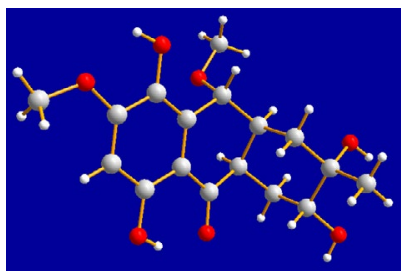

(c)

9a-hydroxydihydrodesoxy bostrycin (42)<smiles>COC1=CC(=O)c2c(O)c3c(c(O)c2C1=O)C[C@](C)(O)[C@H](O)[C@H]3O</smiles>

bostrycin (45)

$44 a$<smiles>COc1cc(O)c2c(c1O)C(=O)c1cc(C)c(O)cc1C2=O</smiles>

3,5,8-trihydroxy-7-methoxy-2methylanthracene-9,10-dione (46)<smiles>COc1cc(O)c2c(c1OC(C)=O)C(=O)c1cc(C)c(O)cc1C2=O</smiles>

$46 a$<smiles>COc1cc(OC(C)=O)c2c(c1O)C(=O)c1cc(C)c(O)cc1C2=O</smiles>

$46 b$<smiles>COc1cc(OC(C)=O)c2c(c1O)C(=O)c1cc(OC(C)=O)c(C)cc1C2=O</smiles>

$46 f$

$46 d$

$46 e$

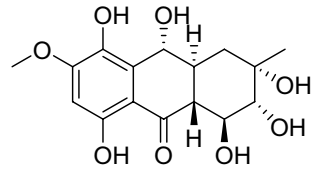

9a-hydroxyhalorosellinia A (43)<smiles>COC1=CC(=O)c2c(O)c3c(c(O)c2C1=O)C[C@@](C)(O)[C@H](OC(C)=O)[C@@H]3O</smiles>

3-acetoxybostrycin (45a)<smiles>COc1cc(O)c2c(c1O)C(=O)c1cc(C)c(OC(C)=O)cc1C2=O</smiles>

$46 c$<smiles>COc1cc(O)c2c(c1OC(C)=O)C(=O)c1cc(C)ccc1C2=O</smiles>

$47 a$

Fig. 10 Anthraquinone derivatives from the sea anemone-derived fungus Nigrospora sp. ZJ-2010006

Fig. 11 Antibacterial structure-activity relationships for hydroanthraquinone derivatives
9-OH might not affect antibacterial Cycloaliphatic ring is very important for activity apparently antibacterial activity

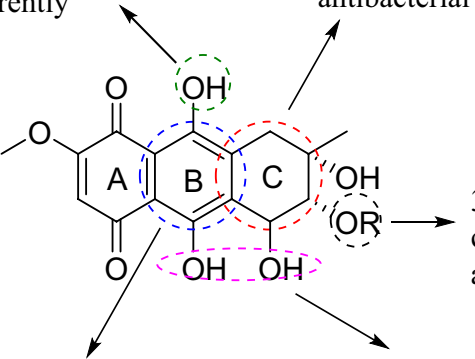

3-OAc in 44a has a positive contribution to antibacterial activity.

Aromatic ring may improve antibacterial activity
4-OH and $10-\mathrm{OH}$ are not essential for antibacterial activity 
<smiles>COc1cc(O)c2c(c1)[C@H](O)[C@@H]1C[C@](C)(O)[C@@H](O)C[C@H]1C2=O</smiles>

tetrahydroaltersolanol C (48)<smiles>COc1cc(O)c2c(c1)[C@H](O)C1C(=C[C@H](O)[C@](C)(O)C1O)C2=O</smiles>

dihydroaltersolanol A (52)<smiles>COc1cc(O)c2c(c1)C(=O)c1c(cc(C)c(O)c1-c1c(OC)cc3c(c1O)C(=O)c1cc(C)c(O)cc1C3=O)C2=O</smiles>

alterporriol Q (56)<smiles>COc1cc(O)c2c(c1)[C@H](O)[C@@H]1C[C@H](C)[C@@H](O)C[C@H]1C2=O</smiles>

tetrahydroaltersolanol D (49)<smiles>COc1cc(O)c2c(c1-c1c(OC)cc(O)c3c1C(=O)C1=C(C[C@H](O)[C@](C)(O)[C@@H]1O)C3=O)C(=O)C1=C(C2=O)[C@@H](O)C(C)(O)[C@@](C)(O)[C@H]1O</smiles>

alterporriol N (53)<smiles>COc1cc(O)c2c(c1)C(=O)c1cc(C)c(O)c(-c3c(OC)cc(O)c4c3C(=O)c3cc(C)c(O)cc3C4=O)c1-2</smiles>

alterporriol R (57)<smiles>COc1cc(O)c2c(c1)[C@H](O)[C@@H]1C[C@](C)(O)[C@@H](O)C[C@H]1C2=O</smiles><smiles>COc1cc(O)c2c(c1)[C@H](O)C1C[C@](C)(O)[C@@H](OC(C)=O)C[C@H]1C2=O</smiles>

tetrahydroaltersolanol E (50)

tetrahydroaltersolanol $\mathrm{F}$ (51)<smiles>C/C=C(\C)OC</smiles>

alterporriol O (54)<smiles>COc1cc2c(c(O)c1-c1c(O)c(C)cc3c1C(=O)c1c(O)cccc1C3=O)C(=O)C1=C(C[C@H](O)[C@@H](O)C1(C)O)C2=O</smiles>

alterporriol $\mathrm{P}$ (55)

Fig. 12 Anthraquinone monomers and dimers from the soft coral-derived fungus Alternaria sp. ZJ-2008003

\section{Alkaloids}

Marine-derived alkaloids represent a class of compounds with particularly privileged structures, which have been frequently encountered in a vast number of pharmaceuticals as well as agrochemicals (Bandini and Eichholzer 2009; Hibino and Choshi 2001; Humphrey and Kuethe 2006; Kochanowska-Karamyan and Hamann 2010; Lounasmaa and Tolvanen 2000). For example, raistrickindole A, an indole diketopiperazine alkaloid obtained from the marine-derived fungus Penicillium raistrickii, displayed antiviral activity against the hepatitis $\mathrm{C}$ virus ( $\mathrm{Li}$ et al. 2019a). Agelastatin A, a bromopyrrole marine alkaloid isolated from the Mexican sponge, Agelas sp., exhibited strong antineoplastic activity (Pettit et al. 2005). Our group has isolated more than 100 alkaloids with promising biological activities from diverse fungal strains derived from marine invertebrates (Chen et al. 2014a; Jia et al. 2015; Shao et al. 2015b; Wang et al. 2015a). To obtain significant antibacterial compounds, efficient and environmental friendly methods for synthesis of unsymmetrical bisindolylmethanes or triarylmethane were developed (Wen et al. 2015).
Compound $103((+)$ - and (-)-pestaloxazine A) (Fig. 16), a pair of new enantiomeric alkaloid dimers with an unprecedented symmetric spiro[oxazinane-piperazinedione] skeleton, were isolated from a soft coral-derived fungus Pestalotiopsis sp. (Jia et al. 2015). A plausible biosynthetic pathway for $\mathbf{1 0 3}$ was proposed starting from two molecules of $L$-ornithine (Fig. 17). The piperazinedione intermediate 103a is produced by double dehydration. The oxidation results in the presence of the $\mathrm{N}$-oxide $\mathbf{1 0 3 b}$, and the key racemic intermediates $( \pm)-\mathbf{1 0 3 c}$ are achieved by intermolecular nucleophilic attack at the same face. The condensation between 103c and 103d gives the final product $( \pm)-\mathbf{1 0 3}$. The epimer $\left(2 R, 2^{\prime} S\right.$ or $\left.2 S, 2^{\prime} R\right)$ of $( \pm)-\mathbf{1 0 3}$, a mesomer, was not observed, indicating that the production of $\mathbf{1 0 3}$ in the fungus was regulated by enzymes with selective catalytic functions. Compound 103 exhibited potent and selective antiviral activity against Enterovirus 71 (EV71) $\left(\mathrm{IC}_{50}=14.2 \pm 1.3 \mu \mathrm{mol} / \mathrm{L}\right)$, 18 -fold more potent than the positive control ribavirin $\left(\mathrm{IC}_{50}=256.1 \pm 15.1 \mu \mathrm{mol} / \mathrm{L}\right)$. Therefore, 103 could be considered as a promising antiviral agent (Jia et al. 2015).

From the gorgonian coral-derived fungus Scopulariopsis sp., six dihydroquinolin-2-one-containing alkaloids were isolated, including three 
<smiles>Cc1cc(O)cc(O)c1C(=O)OC1[C@H]2COC(/C=C/CO)=CC2=CC(=O)[C@](C)(O)[C@@H]1Oc1ccccc1</smiles>

pinophilin B (62)

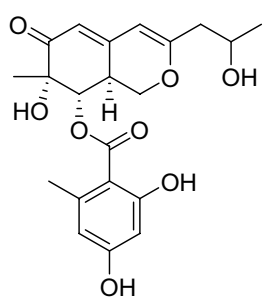

pinophilin E (59)

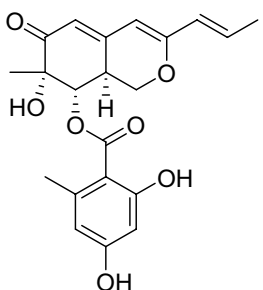

Sch $725680(63)$<smiles>Cc1cc(O)cc(O)c1C(=O)OC1(C)C(=O)C2=C(C=C(/C=C/C(=O)O)OC2)C[C@H]1O</smiles>

pinophilin $\mathrm{F}(60)$

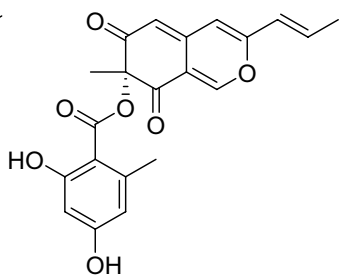

(-)-mitorubrin (64)<smiles>C/C=C/C1=CC2=CC(=O)[C@](C)(O)[C@H](OC(=O)c3c(C)cc(O)cc3O)C2=CO1</smiles>

Sch 1385568 (61)<smiles>Cc1cc(O)cc(O)c1C(=O)OC1(C)C(=O)C=C2C=C(/C=C/CO)OC=C2C1=O</smiles>

(-)-mitorubrinol (65)<smiles>Cc1cc(O)cc(O)c1C(=O)OC1C(=O)C=C2C=C(C=CC(=O)O)OC=C2C1=O</smiles>

Fig. 13 Azaphilone derivatives from the gorgonian-derived fungus P. pinophilum XS-20090E18

Fig. 14 Azaphilone derivatives from the sponge-derived fungus P. sclerotiorum<smiles>CC(=O)/C=C/C1=CC2=C(Cl)C(=O)C(C)(O)[C@H](O)C2CO1</smiles>

penicilazaphilone D (67)<smiles>CCC(C)C(O)C(C)(O)/C=C/C1=CC2=CC(=O)[C@](C)(O)C(O)C2CO1</smiles>

geumsanol C (70)<smiles>CC1CCOC1C(C)(O)/C=C/C1=CC2=C(Cl)C(=O)C(C)(O)[C@H](O)C2CO1</smiles>

penicilazaphilone $\mathrm{E}(\mathbf{6 8})$<smiles>CCC(C)C(O)C(C)(O)/C=C/C1=CC2=C(Cl)C(=O)C(C)(O)C(O)C2CO1</smiles>

WB (71)<smiles>CCC(C)/C=C(C)/C=C/C1=CC2=C(Cl)C(=O)C(C)(OC(C)=O)C(=O)C2=CO1</smiles>

(+)-sclerotiorin (69)<smiles>CC[C@H](C)[C@H](O)[C@@](C)(O)/C=C/C1=CC2=C(Cl)C(=O)[C@](C)(O)[C@H](O)[C@H]2CO1</smiles>

geumsanol G (72)
4-phenyl-3,4-dihydroquinolin-2(1H)-one alkaloids containing a monoterpenoid moiety, 104 (aniduquinolone A), 105 (aflaquinolone A), 106 (aflaquinolone D), and three 4-phenyl-3,4-dihydroquinolin-2(1H)-one alkaloids, 107 (6-deoxyaflaquinolone E), 108 (aflaquinolone F), 109 (aflaquinolone G) (Fig. 18) (Shao et al. 2015b). These dihydroquinolin-2-one-containing alkaloids except 109 exhibited significant anti-larval settlement activity against B. amphitrite. This is the first report on the antifouling activity for quinolin alkaloids. In particular, compound 104 showed highly potent antifouling activity against B. amphitrit at a picomolar level $\left(\mathrm{EC}_{50}=17.5 \mathrm{pmol} / \mathrm{L}\right)$, 249,000 -fold stronger than that of Sea-Nine $211^{\mathrm{TM}}\left(\mathrm{EC}_{50}\right.$
$4.36 \mu \mathrm{mol} / \mathrm{L})$. Particularly, 104 displayed a high therapeutic ratio $\left(\mathrm{LC}_{50} / \mathrm{EC}_{50}=1200\right)$. Therefore, it represents a structural class for discovery of effective, non-toxic, environmentally friendly antifouling agents (Shao et al. 2015b).

From the mycelia of a gorgonian-derived Aspergillus sp. fungus, two new prenylated dihydroquinolone derivatives, 110 (22- $O$-( $N$-Me- $L$-valyl)-aflaquinolone B) and 111 (22-O-(N-Me- $L$-valyl)-21-epi-aflaquinolone B), and two known analogues, 105 and 106 (aflaquinolones A and D) (Fig. 19), were obtained (Chen et al. 2014a). Compounds 110 and $\mathbf{1 1 1}$ possess an unusual esterification of $N$-Me- $L$-Val to the side chain prenyl group. It is worth noting that compound 
<smiles>CCC(C)/C=C(C)/C=C/C1=CC2=C(Cl)C(=O)[C@](C)(OC(C)=O)C(=O)C2=CN1C</smiles>

73<smiles>C#CCN1C=C2C(=O)[C@](C)(OC(C)=O)C(=O)C(Cl)=C2C=C1/C=C/C(C)=C/C(C)CC</smiles>

76<smiles>CCC(C)/C=C(C)/C=C/C1=CC2=C(Cl)C(=O)[C@](C)(OC(C)=O)C(=O)C2=CN1C1CCC1</smiles>

79<smiles>CCC(C)/C=C(C)/C=C/C1=CC2=C(Cl)C(=O)[C@](C)(OC(C)=O)C(=O)C2=CN1O</smiles>

82<smiles>CCC(C)/C=C(C)/C=C/C1=CC2=C(Cl)C(=O)[C@](C)(OC(C)C)C(=O)C2=CN1c1ccc(F)cc1</smiles>

85<smiles></smiles>

88<smiles></smiles>

91<smiles>CCC(C)/C=C(C)/C=C/C1=CC2=C(Cl)C(=O)C([Ge])(OC(C)C)C(=O)C2=CN1c1cccc(C(N)=O)c1</smiles>

94<smiles>[H][Z10]([H])(C)O[C@@]1(C)C(=O)C2=CN(CCC)C(/C=C/C(C)=C/C(C)CC)=CC2=C(Cl)C1=O</smiles>

74<smiles>CCC(C)/C=C(C)/C=C/C1=CC2=C(Cl)C(=O)[C@](C)(OC(C)=O)C(=O)C2=CN1C1CC1</smiles>

77<smiles>CCCCCCN1C=C2C(=O)[C@](C)(OC(C)C)C(=O)C(Cl)=C2C=C1/C=C/C(C)=C/C(C)CC</smiles>

80

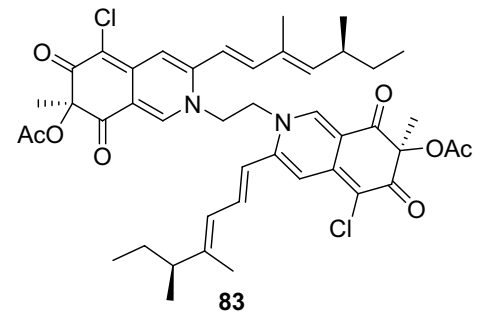<smiles></smiles><smiles></smiles>

75<smiles></smiles>

78<smiles></smiles>

81

86

87<smiles>[C]OC1(C)C(=O)C2=CN(CCc3c[nH]c4ccc(O)cc34)C(/C=C/C(C)=C/C(C)CC)=CC2=C(Cl)C1=O</smiles><smiles>[C]OC1(C)C(=O)C2=CN(c3ccccc3C#N)C(/C=C/C(C)=C/C(C)CC)=CC2=C(Cl)C1=O</smiles><smiles>CCC(C)/C=C(C)/C=C/C1=CC2=C(Cl)C(=O)[C@]([Ge])(OC(C)C)C(=O)C2=CN1c1ccc(C#N)cc1</smiles>

92<smiles>CCC(C)/C=C(C)/C=C/C1=CC2=C(Cl)C(=O)C(C)(OC(C)=O)C(=O)C2=CN1c1ccccc1C(N)=O</smiles>

93<smiles>[C]O[C@@]1(Cl)C(=O)C2=CN(c3ccc(C(N)=O)cc3)C(/C=C/C(C)=C/C(C)CC)=CC2=C(Cl)C1=O</smiles>

95<smiles>CCC(C)/C=C(C)/C=C/C1=CC2=C(Cl)C(=O)C(C)(OC(C)=O)C(=O)C2=CN1c1ccc(NC(C)=O)cc1</smiles>

96

Fig. 15 Semisynthetic azaphilone derivatives from the natural compound (+)-sclerotiorin (69) 
<smiles>CCC(C)/C=C(C)/C=C/C1=CC2=C(Cl)C(=O)C(C)(OC(C)=O)C(=O)C2=CN1c1cccc(C(C)=O)c1</smiles><smiles></smiles>

100<smiles></smiles>

98<smiles>CCC(C)/C=C(C)/C=C/C1=CC2=C(Cl)C(=O)[C@@](C)(OC)OC2=CN1c1ccc(OC)c(OC)c1</smiles>

101<smiles>CCC(C)/C=C(C)/C=C/C1=CC2=C(Cl)C(=O)[C@](C)(OC)C(=O)C2=CN1c1ccc(OC)cc1</smiles>

99<smiles></smiles>

102

Fig. 15 (continued)
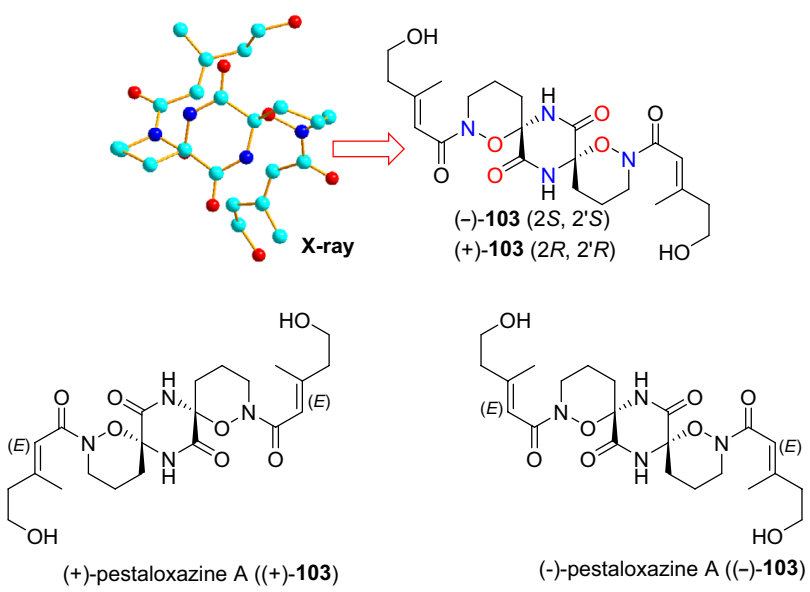

(-)-pestaloxazine A ((-)-103)

Fig. 16 Enantiomeric alkaloid dimers with an symmetric spiro[oxazinane-piperazinedione] skeleton from the soft coral-derived fungus Pestalotiopsis sp.

111 exhibited outstanding anti-RSV (Human respiratory syncytial virus $(\mathrm{RSV}))$ activity $\left(\mathrm{IC}_{50}=42 \mathrm{nmol} / \mathrm{L}\right), 500$ fold more potent than that of ribavirin $\left(\mathrm{IC}_{50}=20 \mu \mathrm{mol} / \mathrm{L}\right.$ ) and showed a comparatively higher therapeutic ratio $\left(\mathrm{TC}_{50} /\right.$ $\mathrm{IC}_{50}=520$ ). This is the first report of prenylated dihydroquinolone derivatives with potent antiviral activity (Chen et al. 2014a).

Eight indol alkaloids were isolated from the bacterium Pseudovibrio denitrificans strain UST4-50 derived from an unidentified ascidian, including a diindol-3-ylmethane (DIM), 112 (di( $1 H$-indol-3-yl)methane), and several analogues, 113 (vibrindole A), 114 (3,3'-di- $1 H$-indol-3-yl1,2-propandiol), 115 (tri(1H-indol-3-yl)methane), 116 (1,2,2-tri(1H-indol-3-yl) ethanone), 117 (arsindoline A), 118 (3, 3'-(phenylmethylene) bis- $1 H$-indole), and 119 (4-(di(1Hindol-3-yl)methyl) phenol (DIM-Ph-4-OH)) (Fig. 20) (Wang et al. 2015a). All DIMs showed low-toxic anti-larval

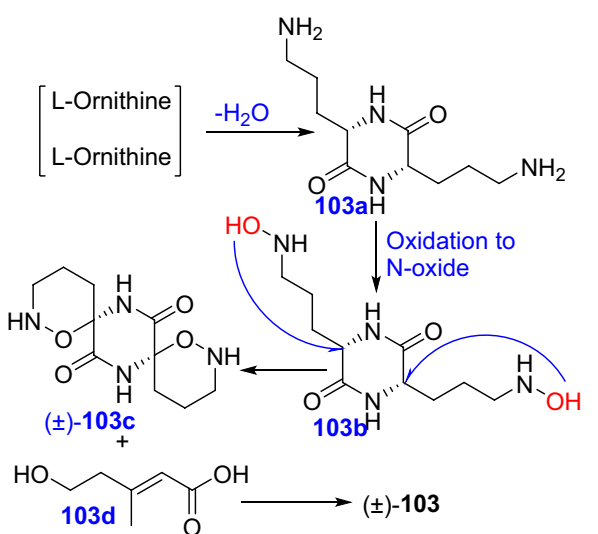

Fig. 17 Plausible biosynthetic pathway to $\mathbf{1 0 3}$

settlement activity against $B$. amphitrite $\left(\mathrm{EC}_{50}=18.57\right.$ to $\left.1.86 \mu \mathrm{mol} / \mathrm{L} ; \mathrm{LC}_{50} / \mathrm{EC}_{50}>15\right)$. Structure-activity relationship analysis revealed that 3,3'-diindolylmethylene and $\mathrm{Ph}-\mathrm{Cl}^{\prime \prime \prime}$ phenolic hydroxyl were necessary for the activity. A field test of $\mathbf{1 1 2}$ over a period of 5 months further confirmed its antifouling activity comparable to Sea-Nine $211^{\mathrm{TM}}$ (Wang et al. 2015a). DIMs could be considered promising candidates as environmentally friendly antifouling agents owing to their simple structures, excellent activities, and low toxicities against marine target organisms.

The bisindole derivatives manifest significant biological activities, while the synthesis method for unsymmetrical bisindolylmethanes or triarylmethanes still remains a great challenge (Abe et al. 2013; Fu et al. 2013; Ma and Yu 2005; $\mathrm{Yu}$ and $\mathrm{Yu}$ 2009; Zhu et al. 2002). In our study, an efficient $\mathrm{S}_{\mathrm{N}} 1$-type reaction was developed for 3-indolylmethanols with miscellaneous nucleophiles, featuring catalyst-free, low cost, wide substrate scope and mild reaction conditions (Figs. 21,22). This approach provides an efficient and environmental friendly method for synthesis of diverse 


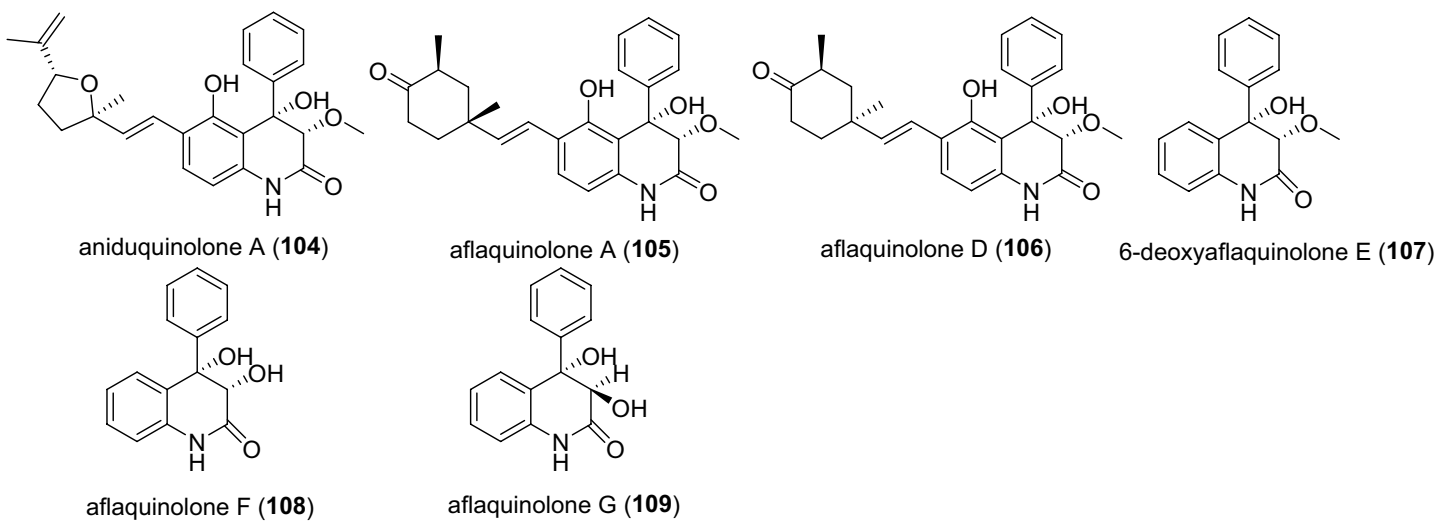

Fig. 18 Dihydroquinolin-2-one-containing alkaloids from the gorgonian coral-derived fungus Scopulariopsis sp.

3-substituted indolyl derivatives as well as unsymmetrical bisindolylmethanes and triarylmethanes (Wen et al. 2015).

As part of our continuous work in this area, versatile 3-substitued indolyl derivatives were synthesized in high yields, including unsymmetrical diarylmethanes and triarylmethanes. Among them, compounds 120 (2-(phenyl(2phenyl-1H-indol-3-yl)methyl)malononitrile), $\mathbf{1 2 1}$ (4-hydroxy-3-(phenyl(2-phenyl-1H-indol-3-yl)methyl)- $2 H$ chromen-2-one), 122 (4-(phenyl(2-phenyl-1H-indol-3-yl) methyl)phenol) and 123 (2-phenyl-3-(phenyl(2,4,6-trimethoxyphenyl)methyl)- $1 H$-indole) (Fig. 23) exhibited antibacterial activities against $B$. megaterium and $M$. lysodeikticus. In particular, 121 and $\mathbf{1 2 2}$ displayed antibacterial activities against $B$. megaterium $(\mathrm{MIC}=13.5$ and $8.36 \mu \mathrm{mol} / \mathrm{L}$, respectively). In addition, 120, 121 and 122 exhibited potent antibacterial activities against $M$. lysodeikticus $(\mathrm{MIC}=2.10$, 3.35 and $4.18 \mu \mathrm{mol} / \mathrm{L}$, respectively). These synthetic compounds could be considered as promising lead compounds for further investigation and application (Wen et al. 2015).

\section{Terpenoids}

Terpenoids constitute a class of broadly active natural products isolated from a diverse range of marine organisms. For example, 11R-methoxy-5,9,13-proharzitrien3-ol, obtained from an endophytic fungus Trichoderma harzianum X-5 derived from the marine brown alga Laminaria japonica, displayed growth inhibition of some marine phytoplankton species (Song et al. 2018). Nakijinol G, a meroterpenoid obtained from a sponge Hyrtios sp. collected from the South China Sea, showed protein tyrosine phosphatase (PTP1B) inhibitory activity (Wang et al. 2017). Trichodermanin C, a diterpenes obtained from a fungal strain Trichoderma harzianum OUPS-111D-4 derived from sponge Halichondria okadai, exhibited significant cytotoxic activity (Yamada et al. 2017). In our ongoing research on the marine invertebrates and their symboitic microorgnisms, 101 terpenoids including 43 new compounds with novel structures were isolated, which exhibited antibacterial, cytotoxic and antifouling activities (Cao et al. 2015, 2017; Li et al. 2012a).

Four new bisabolane-type sesquiterpenoids were separated from the culture of Aspergillus sp. (ZJ-2008004) derived from the sponge Xestospongia testudinaria, including 124 (aspergiterpenoid A), 125 ((-)-sydonol), 126 ((-)-sydonic acid), and 127 ((-)-5-(hydroxymethyl)-2(2',6',6'-trimethyltetrahydro-2H-pyran-2-yl)phenol) (Fig. 24) (Li et al. 2012a). All are optically active compounds. Compound $\mathbf{1 2 5}$ displayed potent inhibitory activity against $S$. albus and M. tetragenus (MIC $=5.00$ and $1.25 \mu \mathrm{mol} / \mathrm{L}$, respectively), and 127 on S. albus and B. subtilis $(\mathrm{MIC}=5.00$ and $2.50 \mu \mathrm{mol} / \mathrm{L}$, respectively). Compound 126 showed strong inhibiting activity against four pathogenic bacteria B. subtilis, Sarcina lutea $(\mathrm{MIC}=2.50 \mu \mathrm{mol} / \mathrm{L})$, E. coli and $M$. tetragenus and uniquely against two marine bacteria ( $V$. parahaemolyticus and V. anguillarum) (Li et al. 2012a).

Eleven new scalarane sesterterpenoids were isolated from the sponge Carteriospongia foliascens, including
Fig. 19 Prenylated dihydroquinolone derivatives from the gorgonian-derived fungus Aspergillus sp.

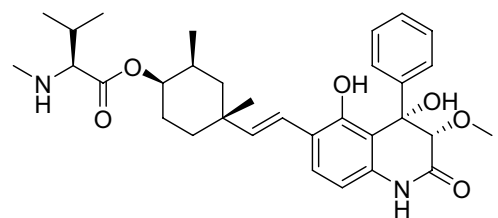

22-O-(N-Me-L-valyl)-aflaquinolone B (110)

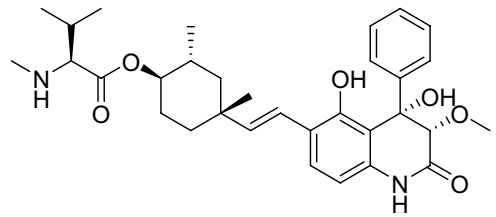

22-O-(N-Me-L-valyl)-21-epi-aflaquinolone B (111) 


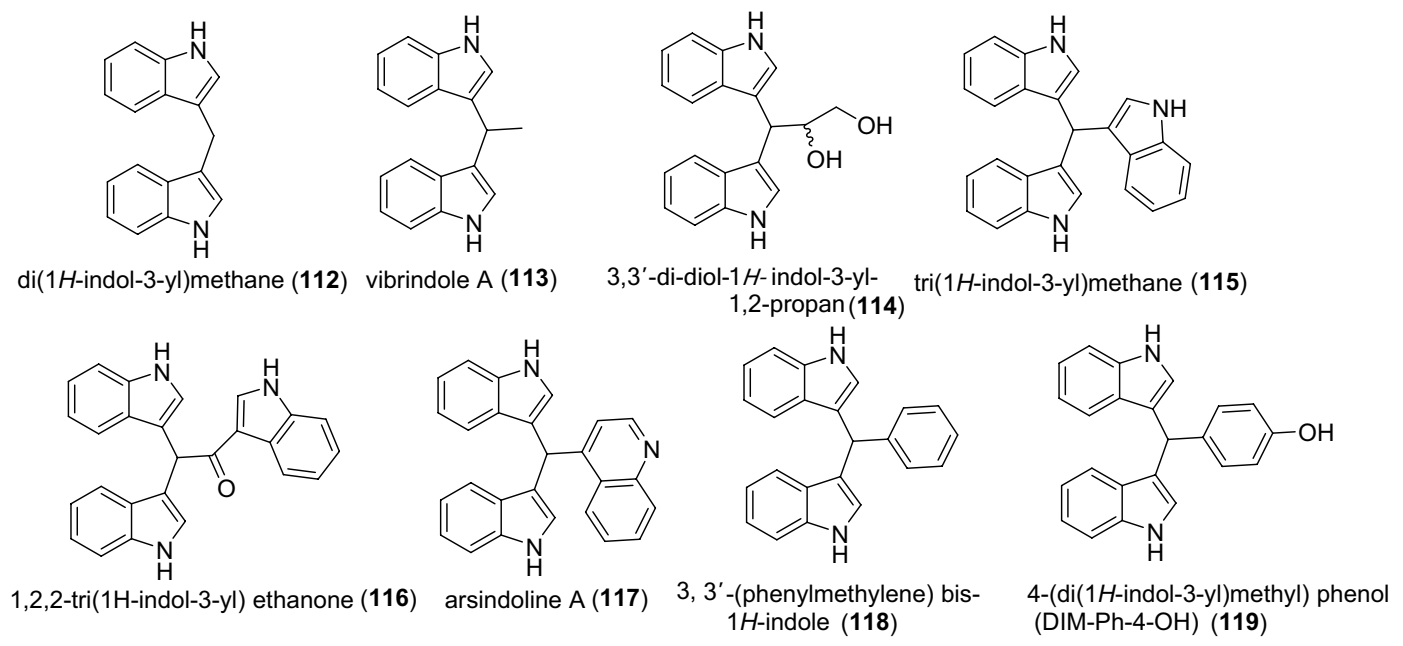

Fig. 20 Diindol-3-ylmethanes from the ascidian-derived bacterium P. denitrificans UST4-50

Fig. 21 Reaction of 3-indolylmethanol with diverse nucleophiles<smiles>[R]n1cc(C(O)O)c2ccccc21</smiles>

$\mathrm{CF}_{3} \mathrm{CH}_{2} \mathrm{OH}$<smiles>[R]N1C=C(C=[Al])C(=CC)c2ccccc21</smiles><smiles>[CH][R]([O-])([O-])n1cc(C(Br)C#N)c2ccccc21</smiles>

catalyst-free high yields diverse nucleophiles broad substrate scope

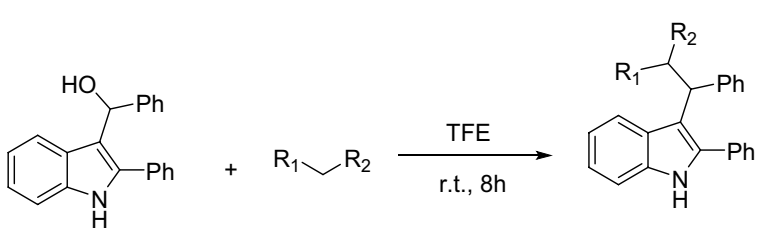

Fig. 22 The active methylene compounds used as nucleophiles

three 20,24-bishomo-25-norscalaranes, 128-130 (carteriofenones A-C), and eight 20,24-bishomoscalaranes, 131-138 (carteriofenones D-K) (Fig. 25) (Cao et al. 2015). Scalarane sesterterpenoids with 4-methylpentanate or pentanoate substituents at the $\mathrm{C}-12$ position were reported for the first time. Compounds 132-135 (carteriofenones $\mathrm{E}-\mathrm{H}$ ) represented rare naturally occurring scalarane sesterterpenoids with a cyclobutane ring. Specifically, the 18-carboxylic scalarane sesterterpenoid 131 displayed strong cytotoxicity against P388, HT-29, and $\mathrm{A} 549$ cell lines $\left(\mathrm{IC}_{50}=0.96,1.43\right.$ and $3.72 \mu \mathrm{mol} / \mathrm{L}$, respectively). Whereas, 130 without the 18 -carboxy was inactive, indicating that the carboxyl group at C-18 might be an indispensable functional group for activity. Additionally, $\mathbf{1 3 1}$ was also found to display brine shrimp lethality towards Artemia salina $\left(\mathrm{LC}_{50}=5.80 \mu \mathrm{mol} / \mathrm{L}\right)$<smiles>N#CC(C#N)C(c1ccccc1)c1c(-c2ccccc2)[nH]c2ccccc12</smiles>

(2-(phenyl(2-phenyl-1Hindol-3-yl)methyl) malononitrile) (120)

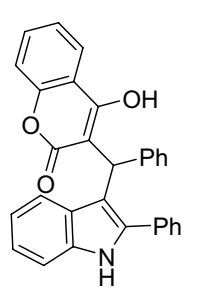

(4-hydroxy-3-(phenyl(2-phenyl$1 \mathrm{H}$-indol-3-yl)methyl)-2Hchromen-2-one) (121)<smiles>Oc1ccc(C(c2ccccc2)c2c(-c3ccccc3)[nH]c3ccccc23)cc1</smiles>

(4-(phenyl(2-phenyl-1H-indol (2-phenyl-3-(phenyl(2,4,6-3-yl)methyl)phenol) (122)

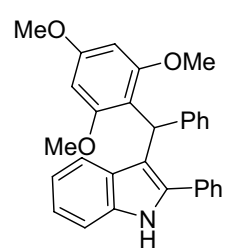

trimethoxyphenyl)methyl)-1 $\mathrm{H}$-indole) (123)

Fig. 23 3-Substitued indolyl derivatives synthesized from di(1H-indol-3-yl)methane 
and anti-larval settlement activity against $B$. amphitrite $\left(\mathrm{EC}_{50}=2.50 \mu \mathrm{mol} / \mathrm{L}\right)(\mathrm{Cao}$ et al. 2015).

From the gorgonian Euplexaura sp. GXWZ-05, three new serrulatane-type diterpenoids, 139-141 (euplexaurenes A-C), and a known metabolite, 142 (anthogorgiene P) (Fig. 26), were isolated (Cao et al. 2017). The absolute configurations of C-11 in 139-142 were difficult to determine by common methods due to the high conformational flexibility of the eight-carbon aliphatic chain attached at C-4. By vibrational circular dichroism (VCD), their absolute configurations were determined. Compounds 139-142 displayed selective cytotoxic activities against the human laryngeal carcinoma (Hep-2) cell line $\left(\mathrm{IC}_{50}=1.95,7.80\right.$, 13.6 and $5.85 \mu \mathrm{mol} / \mathrm{L}$, respectively) (Cao et al. 2017).

Six sesquiterpenoids were obtained from the gorgonian Anthogorgia ochracea, including two new guaiazulenebased analogues, 143 and 144 (ochracenoids A and B), along with four known analogues 145 (1-formylguaiazulene), 146 (1-formyl-4-methyl-7-isopropylazulene), 147 (ketolactone), and 148 (3,8-dimethyl-5-isopropyl-6-formylindenone) (Fig. 27) (Zheng et al. 2014). Compound 143 is a rare guaiazulene-based analogue possessing a unique $\mathrm{C}_{16}$ skeleton. Compound 145 exhibited strong ichthyootoxicity with the antiproliferative effects on several aspects of embryo development in zebrafish Danio rerio, including coagulated eggs $(48 \mathrm{~h})$, notochord malformation $(72 \mathrm{~h})$, and embryo death $(72 \mathrm{~h})$ with the $\mathrm{EC}_{50}$ values of $3.98,6.50$, and $7.39 \mu \mathrm{mol} / \mathrm{L}$, respectively (Zheng et al. 2014).

Six diterpenoids were found from the soft coral Sinularia compacta, including two new prenylgermacrane type diterpenoids, 149-150 (lobophytumins A-B), two new prenyleudesmane type diterpenoids, 151-152 (lobophytumins C-D), and two new spatane type diterpenoids, 153-154 (lobophytumins E-F) (Fig. 28) (Li et al. 2011). Although structures of 149-154 are formally quite different, they are biogenetically related to each other. Sesquiterpene compound $155((-)$-germacrene $\mathrm{D})$ is considered as their common precursor (Faulkner 1984). A plausible biogenetic pathway for 149-154 was proposed (Fig. 29). It is worth noting that prenylgermacrane and prenyleudesmane diterpenes are quite rare in soft coral. This is the first report of spatane type diterpenoids from a soft coral source (Li et al. 2011).
Additionally, multiple known diterpenoids were obtained and showed various bioactivities. From the soft coral Sarcophyton infundibuliforme, four known cembrene diterpenoids were obtained, including 156 (sarcophytol-A), 157 (sarcophytol-A acetate), 158 (sarcophytol-H) and 159 (sarcophytonolid-J) (Wang et al. 2011b) (Fig. 30). These compounds exhibited strong anti-larval settlement activity against $B$. amphitrite $\left(\mathrm{EC}_{50}=2.25,1.75,8.13\right.$ and $7.50 \mu \mathrm{g} /$ $\mathrm{mL}$, respectively) (Wang et al. 2011b). From a soft coral Sarcophyton sp., a cembranoid diterpene $\mathbf{1 6 0}$ (sarcophytol B) was isolated. This compound displayed antibacterial activity against $B$. cereus, $S$. albus and $V$. parahaemolyticus $(\mathrm{MIC}=3.13,1.56$, and $0.50 \mu \mathrm{mol} / \mathrm{L}$, respectively) (Cao et al. 2013). Briarane type diterpenoids 161 (juncin $P$ ) and 162 (junceellolide D) isolated from the gorgonian Dichotella fragilis (Ridleg) exhibited potent antifouling activity $\left(\mathrm{EC}_{50}=0.80\right.$ and $0.77 \mu \mathrm{g} / \mathrm{mL}$, respectively) (Zhou et al. 2011).

\section{Steroids}

Steroid derivatives from marine organisms are noted for diverse unusual structures with multiple potent biological properties. For instance, petasitosterone B, a steroid isolated from a Formosan marine soft coral Umbellulifera petasites exhibited promising anti-inflammatory activity (Huang et al. 2016). Two 9,11-secosteroidal glycosides, sinularosides A and B, isolated from the South China Sea soft coral Sinularia humilis, exhibited potent antimicrobial activity (Sun et al. 2012). In our previous reports, 86 steroidal compounds were obtained from marine invertebrates and their symbiotic fungi, which exhibited cytotoxic, antiviral and antibacterial activities (Cao et al. 2014; Sun et al. 2015; Zhao et al. 2013).

From the gorgonian Echinogorgia rebekka, four new steroids with an acetoxy linked at the end of the side chain, 163-166 (echrebsteroids A-D) (Fig. 31) were isolated (Cao et al. 2014). Among them, 164 and 165 were a pair of new C-25 epimers of 26-acetoxy steroids, representing the first reported separation of C-25 epimers of 26-acetoxy steroids. Compound 165 exhibited pronounced antiviral activity against $\mathrm{RSV}\left(\mathrm{IC}_{50}=0.19 \mu \mathrm{mol} / \mathrm{L}\right)$ and a comparatively higher therapeutic ratio $\left(\mathrm{TC}_{50} / \mathrm{IC}_{50}=128\right)$, suggesting that

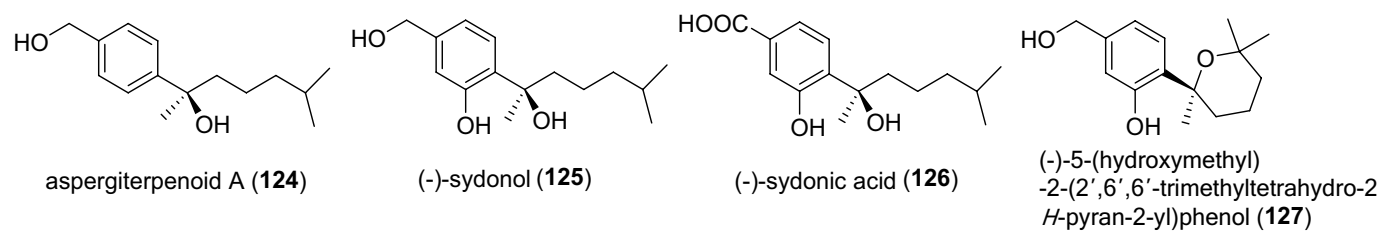

Fig. 24 Bisabolane-type sesquiterpenoids from the sponge-derived fungus Aspergillus sp. 
Fig. 25 Scalarane sesterterpenoids from the sponge $C$. foliascens
Fig. 26 Serrulatane-type diterpenoids from the gorgonian Euplexaura sp. GXWZ-05

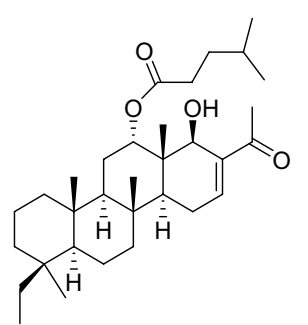

carteriofenone A (128)

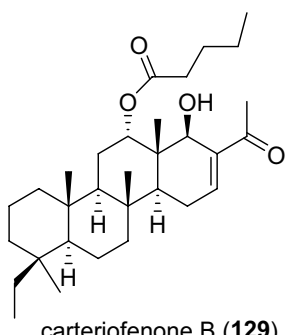

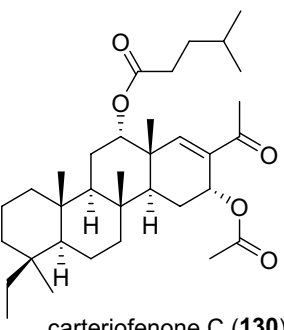

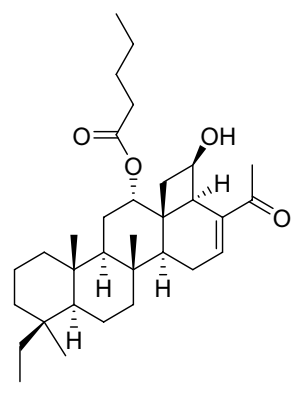

carteriofenone $F(133)$<smiles>CCC(O)CC(=O)O[C@H]1C[C@@H]2C3(C)CCCC(C)(CC)[C@@H]3CCC2(C)[C@@H]2C1=CC(=O)C1=C2C(=O)O[C@]1(C)O</smiles>

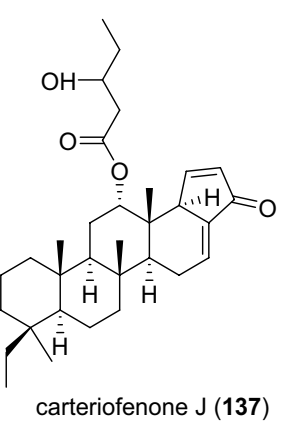

carteriofenone G (134)

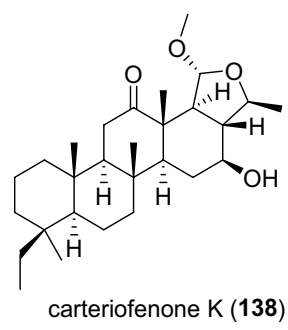

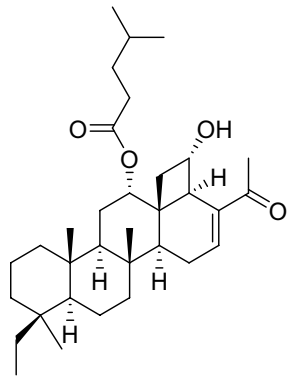

carteriofenone C (130)

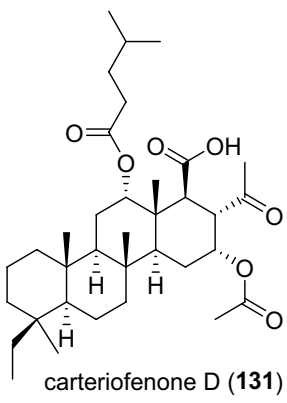

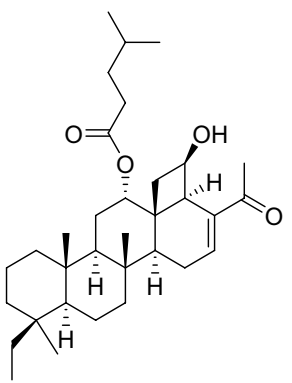

carteriofenone $\mathrm{H}$ (135)

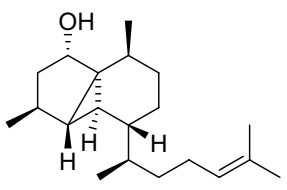

euplexaurene A (139)<smiles></smiles>

euplexaurene B (140)<smiles>CC(C)=C/C=C/C(C)[C@H]1CCC(C)C23CC(=O)C=C(C)[C@H]2[C@H]13</smiles>

euplexaurene $\mathrm{C}$ (141)<smiles>CC(C)=CCCC(C)[C@H]1CCC(C)C23CC12C(C)C(C)=CC3=O</smiles>

anthogorgiene $\mathrm{P}$ (142)
Fig. 27 Guaiazulene-based analogues from the gorgonian A. ochracea<smiles>Cc1cc2c(cc3c(C)c(C=O)cc1-3)[C@H](C)CO2</smiles><smiles>CC1=CC(=O)c2c(C)cc(C(=O)C(C)C)cc21</smiles>

ochracenoid A (143)<smiles></smiles>

ketolactone (147) ochracenoid B (144)<smiles>Cc1ccc(C=O)c2c(C)cc(C(C)C)cc12</smiles>

1-formylguaiazulene (145)<smiles>CC1=CC(=O)c2c1cc(C(C)C)c(C=O)c2C</smiles>

3,8-dimethyl-5-isopropyl-6-

formylindenone (148)

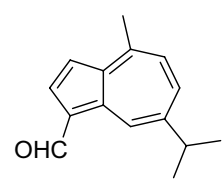

1-formyl-4-methyl-7isopropylazulene (146) 
it could be considered as a potential antiviral agent (Cao et al. 2014).

From a gorgonian Carijoa sp., four pregnane steroid were isolated, including a new pregnane steroid, 167 (15 $\beta$-hydroxypregna-1,4,20-trien-3-one), and three known analogues 168 (15 $\beta$-acetoxypregna1,4,20-trien3-one), 169 (18-acetoxypregna-1,4,20-trien-3-one), and
170 (pregna-1,4,20-trien3-one) (Fig. 32), all with rare 3-one dienones (Zhao et al. 2013). Compounds 167, 169 and 170 displayed cytotoxicity against human hepatoma cell line Bel-7402 $\left(\mathrm{IC}_{50}=9.33,11.02\right.$ and $18.68 \mu \mathrm{mol} / \mathrm{L}$, respectively). Additionally, 167 exhibited potent antibacterial activity against $S$. aureus, S. albus, E. coli, V. parahaemolyticus and Nocardia brasiliensis (MIC $=0.063,1.00$,<smiles>C=C1CCC=C2CC[C@H](C(C)CC(=O)C=C(C)C)CC2C1</smiles><smiles>C=C1CCC=C2CC[C@H](C(C)CC(=O)CC(C)C)C=C1C2</smiles><smiles>C=C1CCC[C@H]2CC[C@@H](C(=C)CCC=C(C)C)C[C@H]12</smiles>

lobophytumin C (151)<smiles>C=C(CCC=C(C)C)[C@H]1CC[C@]2(C)CCC=C(C)[C@H]2C1</smiles>

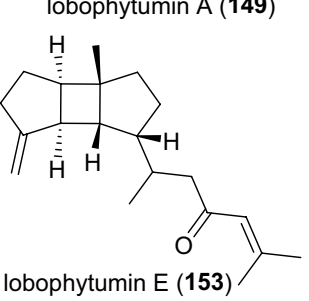

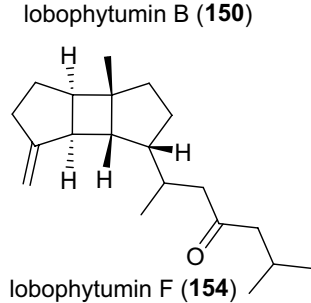<smiles>C=C1/C=C/C(C(C)C)CC/C=C\CC1</smiles>

(-)-germacrene D (155)

Fig. 28 Diterpenoids from the soft coral S. compacta

Fig. 29 Plausible biosynthetic correlations between three extended sesquiterpenoid skeleton diterpenoids

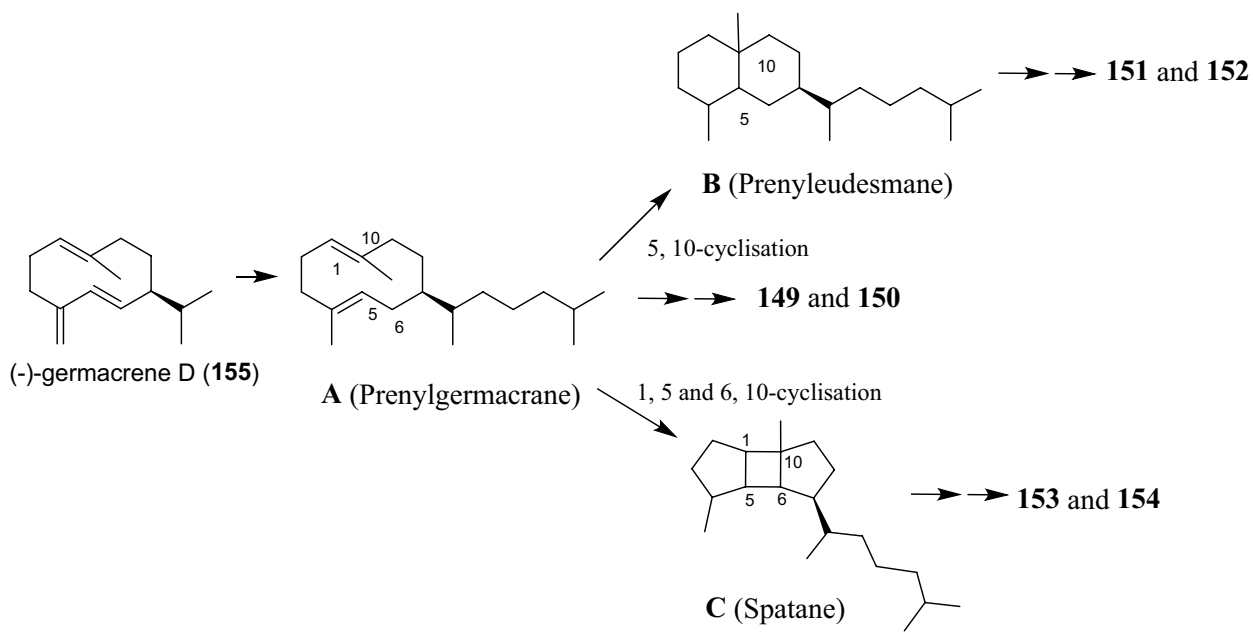

Fig. 30 Cembrene diterpenoids and briarane diterpenoids from the soft coral $S$. infundibuliforme and Sarcophyton sp. and gorgonian D. fragilis

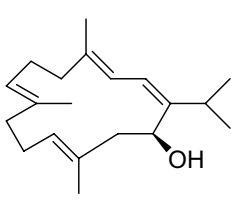

sarcophytol-A (156)

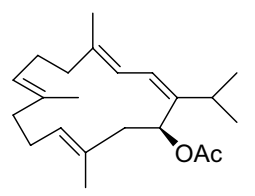

sarcophytol-A acetate (157)
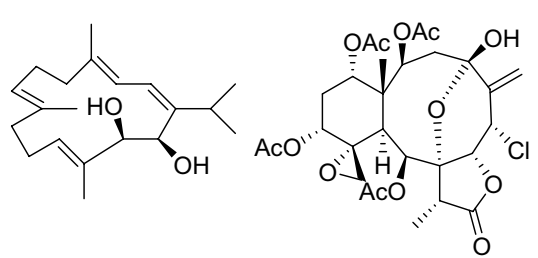

sarcophytol B (160)

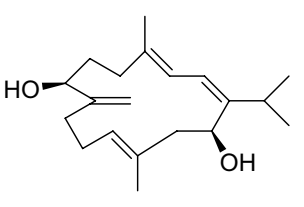

sarcophytol-H (158)

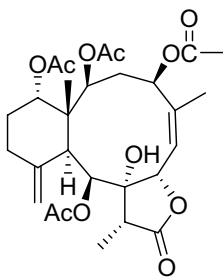

junceellolide D (162)

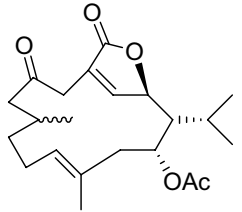

sarcophytonolid-J (159) 
$1.00,4.00$, and $0.500 \mu \mathrm{mol} / \mathrm{L}$, respectively), and displayed excellent inhibitory activity against Pseudomonas putida $(\mathrm{MIC}=31 \mathrm{nmol} / \mathrm{L})$, fivefold stronger than that of ciprofloxacin $(\mathrm{MIC}=156 \mathrm{nmol} / \mathrm{L})$. Compound 168 showed very strong antibacterial activity against pathogenic bacteria Tetragenococcus halophilus $(\mathrm{MIC}=312 \mathrm{nmol} / \mathrm{L})$. While 169 demonstrated strong antibacterial activity against $B$. cereus, $S$. aureus and T. halophilus (MIC $=2.50,0.156$, and $1.25 \mu \mathrm{mol} / \mathrm{L}$, respectively) (Zhao et al. 2013).

From the gorgonian Subergorgia rubra, sixteen secosteroids were obtained, including twelve new 9,10-secosteroids 171-182 (subergorgiaols A-L), along with four known analogues 183 (astrogorgiadiol), 184 (calicoferol C), 185 (calicoferol F), 186 (calicoferol A) (Fig. 33) (Sun et al. 2015). Among them, compounds 171-182, with a series of different aliphatic side chains, represent the first examples of 9,10-secosteroids bearing a hydroxy group at $\mathrm{C}-8$, which are 8-OH derivatives of astrogorgiadiols/ calicoferols. Compound $\mathbf{1 7 4}$ showed selective strong cytotoxicity against the cervical carcinoma cell line (CaSki) $\left(\mathrm{IC}_{50}=2.4 \mu \mathrm{mol} / \mathrm{L}\right)$. Additionally, 176 displayed toxicity toward brine shrimp A. salina $\left(\mathrm{LC}_{50}=2.0 \mu \mathrm{mol} / \mathrm{L}\right)(\mathrm{Sun}$ et al. 2015).

Four polyoxygenated sterols were separated from a soft coral Sinularia sp., including $187((3 S, 23 R, 24 S)$ ergost-5-ene-3 $\beta, 23 \alpha, 25$-triol), 188 ((24S)-ergostane6 -acetate- $3 \beta, 5 \alpha, 6 \beta, 25$-tetraol $), \quad \mathbf{1 8 9}((24 S)$ ergostane-6-acetate- $3 \beta, 6 \beta, 12 \beta, 25$-tetraol) and $\mathbf{1 9 0}$ (24-methylenecholestane-3 $\beta, 5 \alpha, 6 \beta$-triol-6-monoacetate) (Fig. 34) (Li et al. 2012b). Compound 190 showed moderate cytotoxicity against the $\mathrm{K} 562$ cell line $\left(\mathrm{IC}_{50}=3.18 \mu \mathrm{mol} / \mathrm{L}\right)$ and also demonstrated potent lethality toward brine shrimp A. salina $\left(\mathrm{LC}_{50}=0.96 \mu \mathrm{mol} / \mathrm{L}\right)$.

Chemical investigation of sponge Topsentia sp. led to the isolation of three novel polyhydroxylated sterol derivatives, 191-193 (topsensterols A-C), with novel $2 \beta, 3 \alpha, 4 \beta, 6 \alpha$-tetrahydroxy- $14 \alpha$-methyl $\Delta^{9(11)}$ steroidal core and rare side chains (Fig. 35) (Chen et al. 2016). The differences between them were mainly existed in the respective aliphatic side chains. Compound 192 demonstrated significant cytotoxicity against human gastric carcinoma cell line SGC-7901 $\left(\mathrm{IC}_{50}=8.0 \mu \mathrm{mol} / \mathrm{L}\right)$. While 193
Fig. 31 Steroids with 26-acetoxy from the gorgonian $E$. rebekka
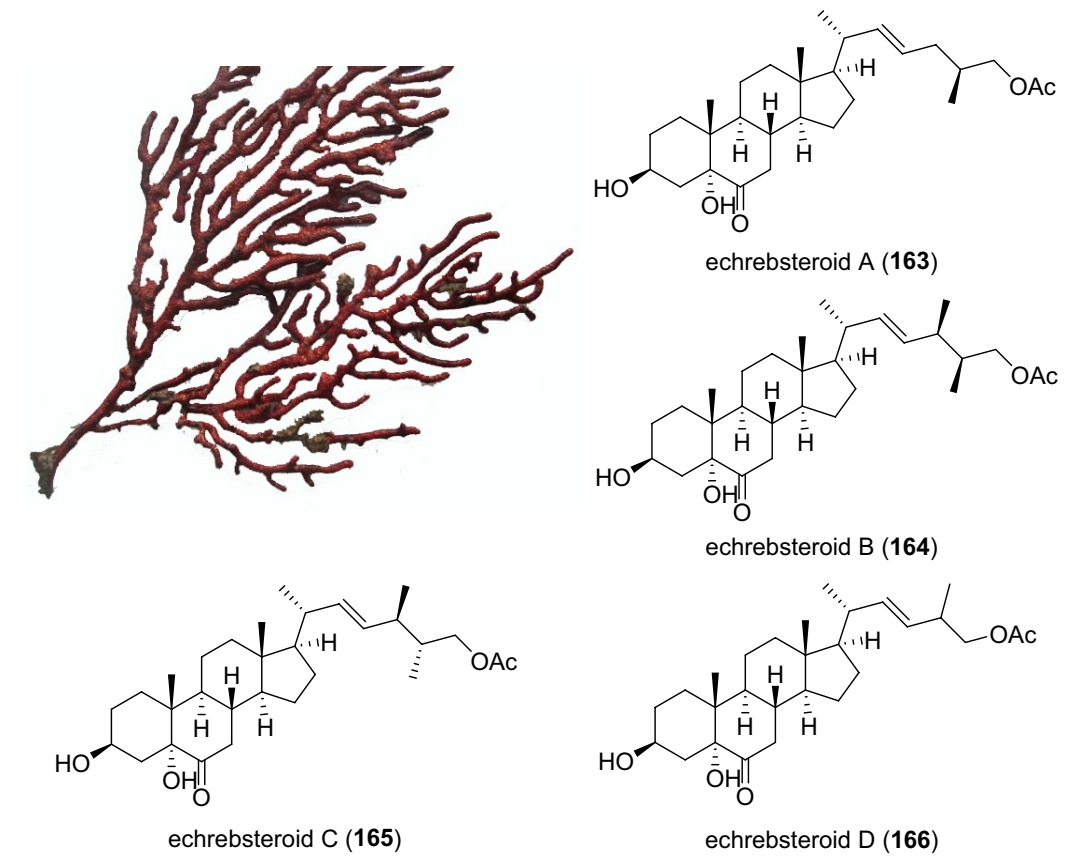
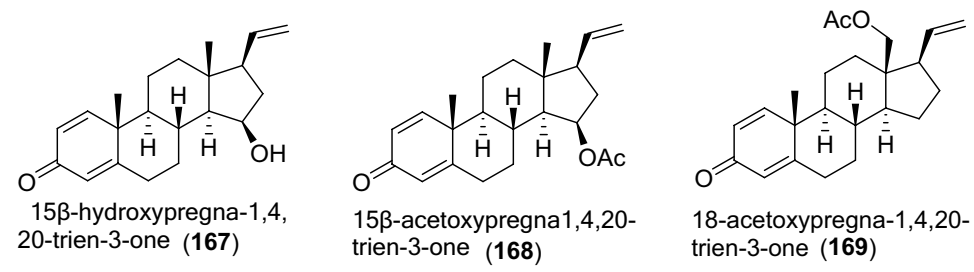

18-acetoxypregna-1,4,20trien-3-one (169)

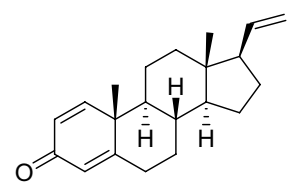

pregna-1,4,20-trien3-one (170)

Fig. 32 Pregnane steroids from the gorgonian E. rebekka 
showed cytotoxicity against human erythroleukemia cell line $\mathrm{K} 562\left(\mathrm{IC}_{50}=6.0 \mu \mathrm{mol} / \mathrm{L}\right)$ (Chen et al. 2016).

Six steroids and one steroidal glycoside were isolated from the fungal strain Cladosporium sp. WZ-2008-0042 separated from the gorgonian Dichotella gemmacea, including a new pregnane, 194 ( $3 \alpha$-hydroxy-7-ene-6,20dione), five known steroids $\mathbf{1 9 5}$ ( $5 \alpha, 8 \alpha$-epidioxy-ergosta6,9,22E-triene $3 \beta$-ol), 196 ( $5 \alpha, 8 \alpha$-epidioxy-ergosta-6,22Edien-3 $\beta$-ol), 197 (ergosta-7,22E-diene- $3 \beta, 5 \alpha, 6 \beta$-triol), 198

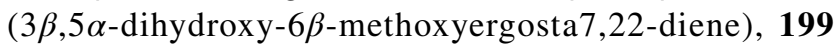
(ergosterol peroxide), along with one known steroidal glycoside 200 (stigma-5-en-3-O- $\beta$-glucopyranoside) (Fig. 36) (Yu et al. 2018). Compounds 194-196 and 198 exhibited potential antiviral activity against $\mathrm{RSV}$ virus $\left(\mathrm{IC}_{50}=0.11\right.$ to $0.17 \mathrm{mmol} / \mathrm{L} ; \mathrm{TC}_{50} / \mathrm{IC}_{50}=5.18$ to 9.92 ).

\section{Phenylpropanoids}

Phenylpropanoids contain many chemical structure types, such as chromone, coumarin, flavone and lignin, which possess various biological activities including antioxidant, cytotoxic, antimicrobial, and enzyme inhibitory activities. For example, arthone $\mathrm{C}$, a chromone derivative isolated from a deep-sea-derived fungus Arthrinium sp., exhibited potent antioxidant activity (Bao et al. 2018). Two tetracyclic coumarin derivatives and two coumarin dimers isolated from the marine-derived fungus Eurotium rubrum showed significant tyrosinase inhibitory activity (Kamauchi et al. 2018). In our previous studies, 80 phenylpropanoid compounds with 54 new compounds were obtained, which showed antifouling, antibacterial and cytotoxic activities (Qi et al. 2013; Zhao
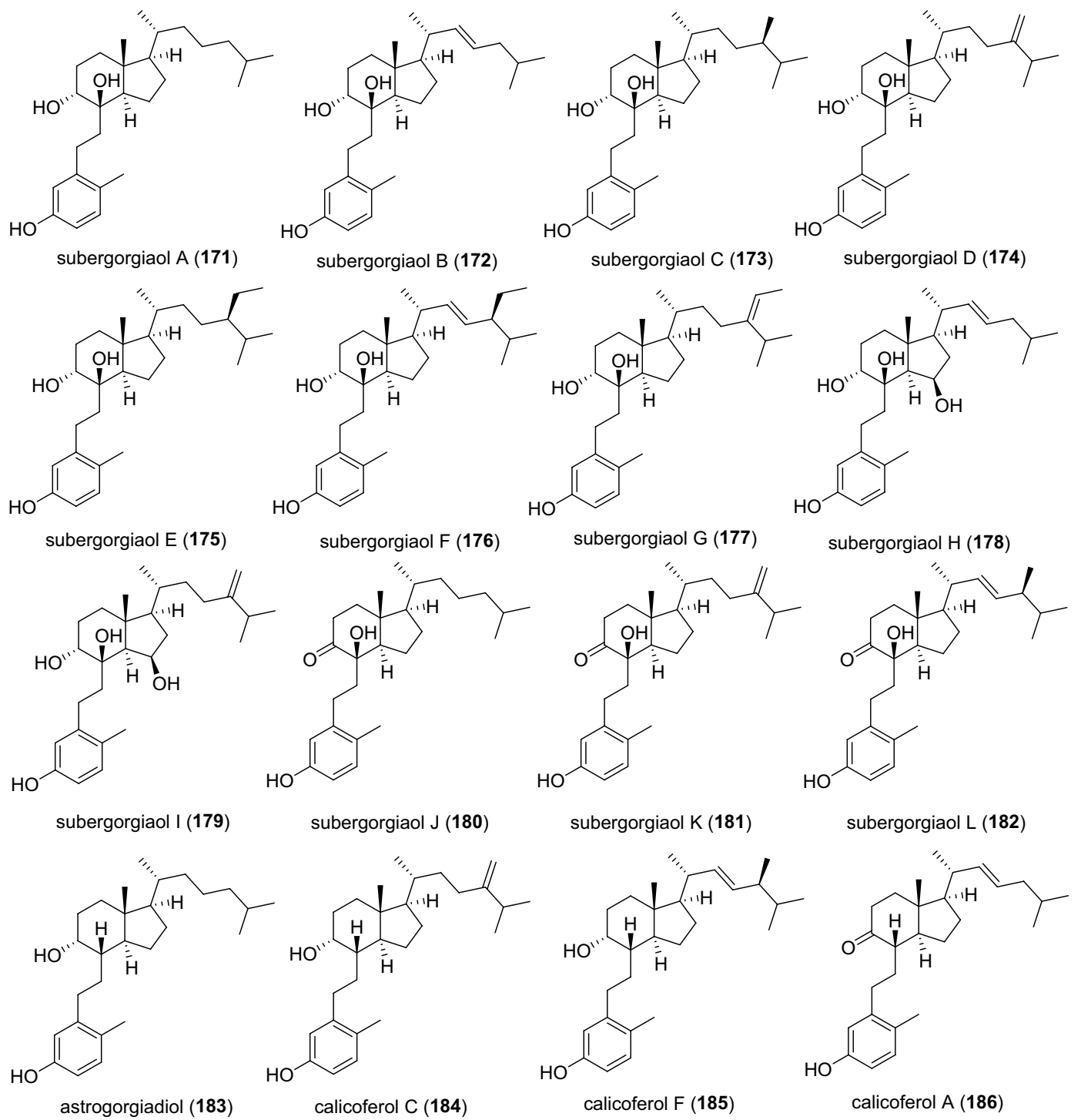

Fig. 33 9,10-Secosteroids from the gorgonian S. rubra 
Fig. 34 Polyoxygenated sterols from the soft coral Sinularia sp.

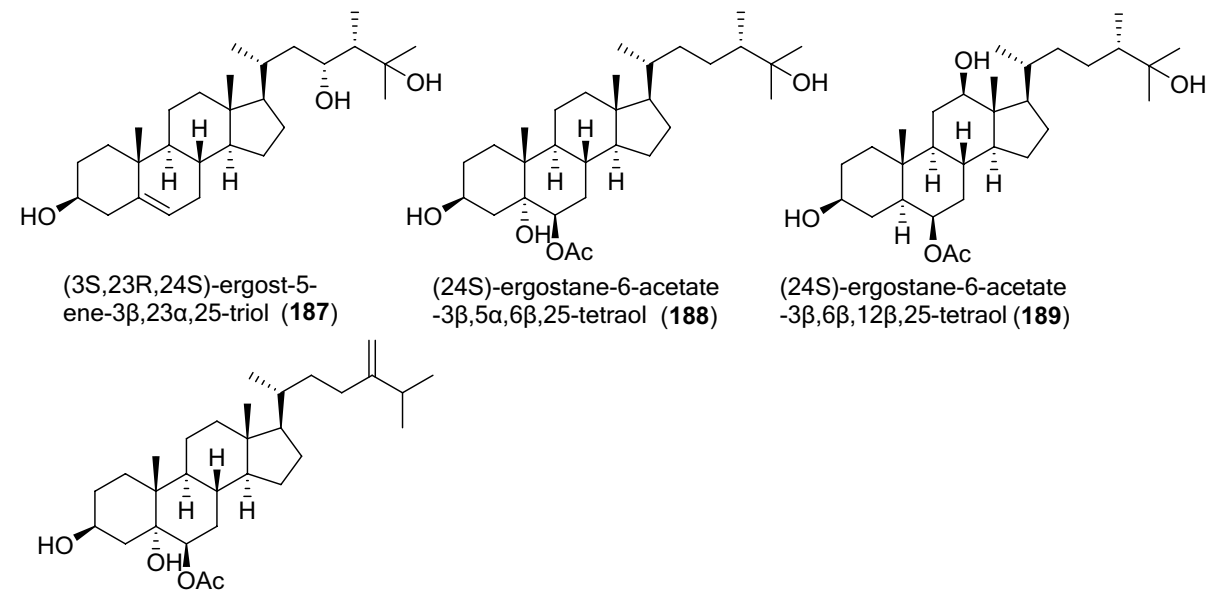

24-methylenecholestane-3 $\beta, 5 \alpha, 6 \beta$-triol-6monoacetate (190) et al. 2015b). These findings provide further insight into the chemical diversity and biological activities of this class of MNPs.

Ten isocoumarins were isolated from the sponge-derived fungus Penicillium sp. MWZ14-4, including three new hydroisocoumarins, 201-203 (penicimarins A-C), three new isocoumarins, 206-208 (penicimarins D-F), together with four known isocoumarin derivatives 204 (aspergillumarin B), 205 (aspergillumarin A), 209 (sescandelin B), and $\mathbf{2 1 0}$ (5,6,8-trihydroxy-4-(1'-hydroxyethyl)isocoumarin) (Fig. 37) (Qi et al. 2013). Compound 201 represents a rare naturally occurring isocoumarin derivative with 4-substitution but no substituent at the 3-position.

Twelve new chromone derivatives, 211-222 (corynechromones A-L) (Fig. 38), were obtained from the spongederived fungus Corynespora cassiicola XS-200900I7 (Zhao et al. 2015b). These are the first chromone derivatives reported from the genus Corynespora. The absolute configurations of 211-220 were determined by the modified Mosher's method and TDDFT ECD calculations along with comparison of their CD spectra. Interestingly, 211/212, 213/214, 215/216, and 217/218 were pairs of epimers at C-2. A biogenetic pathway of the isolated chromone derivatives was proposed (Fig. 39). The different cyclization pathways from an uncyclized $\alpha, \beta$-unsaturated ketone precursor may lead to production of the octalactones (Ebrahim et al. 2012,
2013). Alternatively, the decalactones may be produced by ester formation with the distal hydroxy group. A possible non-enzymatic cyclization route may be involved to form the epimeric pairs. A Michael reaction can occur with the nucleophile attacking from either face, leading to the formation of the epimeric pairs (Zhao et al. 2015b).

From a gorgonian-derived fungus Eurotium sp. XS200900E6, five pairs of new dihydroisocoumarin enantiomers, 223-232 ( \pm )-eurotiumides A-E), together with two related racemates, $\mathbf{2 3 3}$ and $\mathbf{2 3 4}(( \pm)$-eurotiumides $\mathrm{F}$ and $\mathrm{G})$, were isolated, all of them are rare dihydroisocoumarin derivatives with a methoxy at C-4 (Fig. 40) (Chen et al. 2014b). The (+)- and (-)-eurotiumides B and D with cis configurations of $\mathrm{H}-3 / \mathrm{H}-4$ demonstrated significant anti-larval settlement activity against $B$. amphitrite $\left(\mathrm{EC}_{50}=0.7\right.$ to $\left.2.3 \mu \mathrm{g} / \mathrm{mL} ; \mathrm{LC}_{50} / \mathrm{EC}_{50}>15\right)$, indicating that they could be considered as promising environmentally benign antifouling agents. Additionally, 223, 224, 225, and 226 displayed significant antibacterial activities against $S$. epidermidis (MICs $=0.39$ to $0.78 \mu \mathrm{mol} / \mathrm{L}$ ), and $\mathbf{2 2 3}$ and $\mathbf{2 2 4}$ showed remarkable activities against $B$. cereus (MICs $=0.39$ and $0.78 \mu \mathrm{mol} / \mathrm{L}$, respectively). All of the tested compounds, especially $\mathbf{2 2 3}$ and 224, exhibited strong inhibitory activities against two marine bacteria, $V$. anguillarum and V. parahaemolyticus (Chen et al. 2014b).
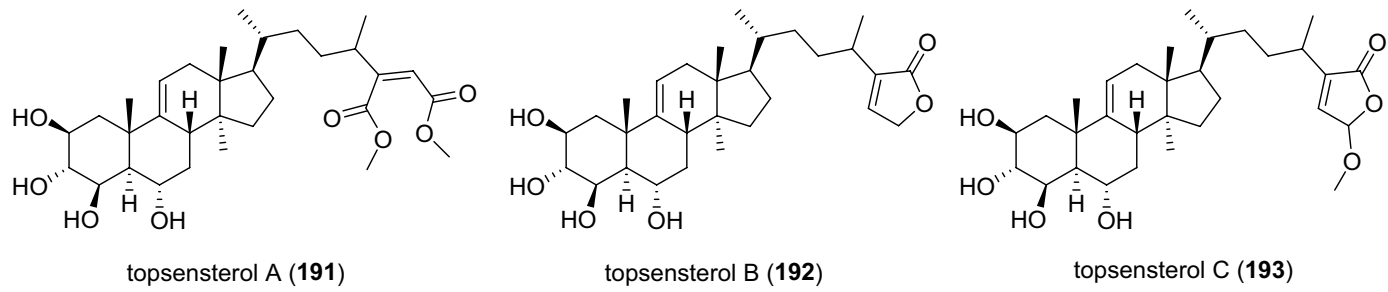

Fig. 35 Polyhydroxylated sterol derivatives from the sponge Topsentia sp. 
Fig. 36 Steroids and steroidal glycoside from the gorgonianderived fungus Cladosporium sp. WZ-2008-0042
Fig. 37 Isocoumarins from the sponge-derived fungus Penicillium sp. MWZ14-4<smiles></smiles>

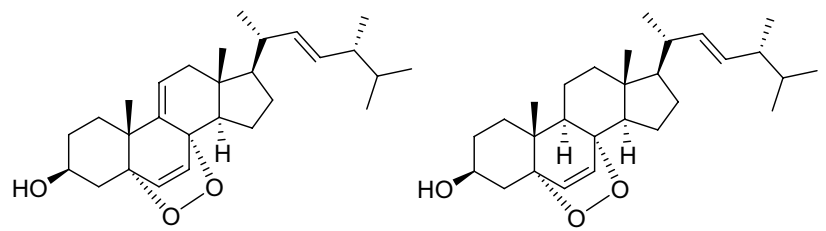

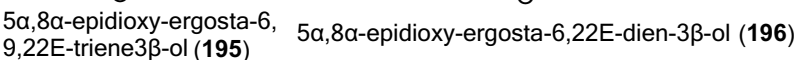<smiles>CC(C)[C@H](C)/C=C/[C@H](C)C1CCC2C3=CC=C4C[C@@H](O)CC[C@]4(C)[C@H]3CCC2(C)[C@H]1C</smiles>

$3 \beta, 5 \alpha$-dihydroxy- $6 \beta$-methoxye rgosta7,22-diene (198)

ergosterol (199)

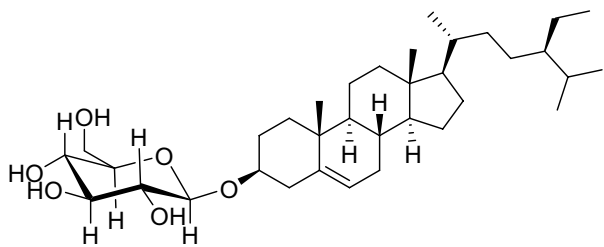

stigma-5-en-3-O-ß-glucopyranoside (200)<smiles>C[C@H](O)[C@H]1COC(=O)c2c(O)cc(O)cc21</smiles><smiles>COc1cccc2c1C(=O)OC(CCCC(C)O)C2</smiles><smiles>COc1c(O)ccc2c1C(=O)OC(CCCC(C)O)C2</smiles>

penicimarin $\mathrm{A}(\mathbf{2 0 1})$ penicimarin C (203)<smiles>CC(O)CCCC1Cc2cccc(O)c2C(=O)O1</smiles>

aspergillumarin $B(204)$<smiles>O=c1oc(CO)c(CO)c2cc(O)cc(O)c12</smiles>

aspergillumarin $\mathrm{A}(\mathbf{2 0 5})$

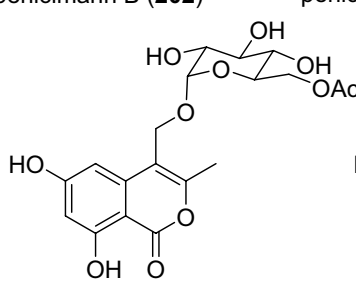

penicimarin $D(206)$<smiles>CC(=O)OCc1c(C)oc(=O)c2c(O)cc(O)cc12</smiles>

penicimarin $E(\mathbf{2 0 7})$<smiles>Cc1oc(=O)c2c(O)cc(O)cc2c1CO</smiles>

sescandelin B (209)<smiles>CC(O)c1coc(=O)c2c(O)c(O)cc(O)c12</smiles>

5,6,8-trihydroxy-4-(1'-hydroxyethyl) isocoumarin (210)

\section{Peptides}

Peptides isolated from many marine species present various biological activities, such as antimicrobial, antiviral, antitumor, antioxidative and cardioprotective activities. For instance, mirabamide A, a cyclic depsipeptide obtained from the sponge Siliquariaspongia mirabilis, showed potent inhibitory activity on HIV-1 fusion (Plaza et al. 2007).
Reniochalistatin E, an octapeptide isolated and characterized from the marine sponge Reniochalina stalagmitis, displayed significant cytotoxic activity (Zhan et al. 2014). In our studies, a total of 34 peptides were identified, of which more than half are cyclopeptides involved in 18 new peptides (Chen et al. 2014c; Hou et al. 2019b, 2019c). Particularly, LC-MS/MS-dependent molecular networking and ${ }^{1} \mathrm{H}$ NMR techniques were applied in order to identify new peptides. 
<smiles>CCOC(=O)Cc1cc(O)cc2c1C(=O)C[C@@H](C[C@H](C)O)O2</smiles>

corynechromone A (211)<smiles>CC(O)C[C@@H]1CC(=O)c2c(CC(=O)O)cc(O)cc2O1</smiles>

corynechromone E (215)<smiles>CC(=O)C[C@H]1CC(=O)c2c(CC(=O)O)cc(O)cc2O1</smiles>

corynechromone I (219)<smiles>CCOC(=O)Cc1cc(O)cc2c1C(=O)C[C@@H](CC(C)O)O2</smiles>

corynechromone B (212)<smiles>CC(O)C[C@H]1CC(=O)c2c(CC(=O)O)cc(O)cc2O1</smiles>

corynechromone $F$ (216)<smiles>CCOC(=O)Cc1cc(O)cc2oc(/C=C/C[C@H](C)O)cc(=O)c12</smiles>

corynechromone J (220)<smiles>CC(C)COC(=O)Cc1cc(O)cc2c1C(=O)C[C@@H](C[C@H](C)O)O2</smiles>

corynechromone C (213)<smiles>COc1c(O)cc(CC(=O)O)c2c1O[C@H](CC(C)O)CC2=O</smiles>

corynechromone G (217)<smiles>CCOC(=O)Cc1cc(O)cc2oc(C)cc(=O)c12</smiles>

corynechromone K (221)<smiles>CC(C)COC(=O)Cc1cc(O)cc2c1C(=O)C[C@@H](CC(C)O)O2</smiles>

corynechromone D (214)<smiles>COc1c(O)cc(CC(=O)O)c2c1O[C@H](CC(C)O)CC2=O</smiles>

corynechromone $\mathrm{H}$ (218)<smiles>Cc1cc(=O)c2c(CC(=O)OCC(C)C)cc(O)cc2o1</smiles>

corynechromone L (222)

Fig. 38 Chromone derivatives from the sponge-derived fungus C. cassiicola XS-200900I7

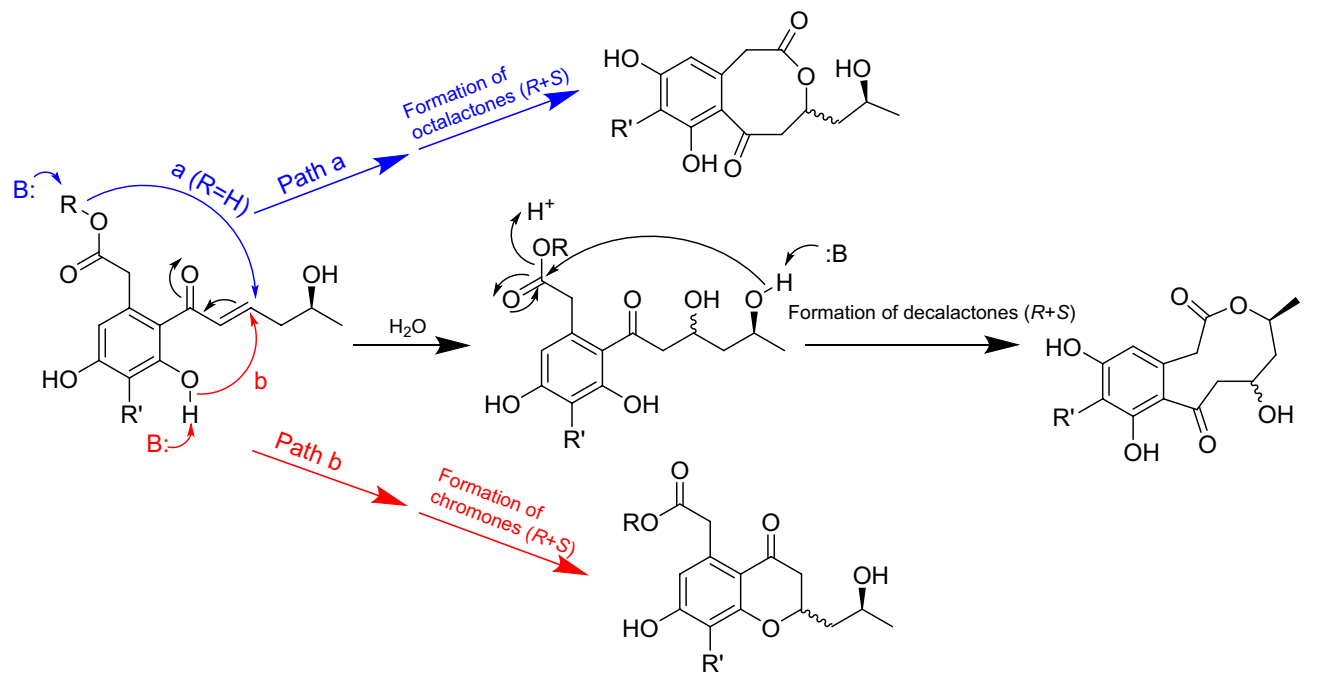

Fig. 39 Proposed biogenetic pathway for corynechromones, octalactones and decalactones

The strategy of molecular networking based on MS/ MS cheminformatics could shed light on structural relationships and accelerate the dereplication of molecules in crude extracts to mine new molecules. Based on integrating LC-MS/MS-dependent molecular networking and ${ }^{1} \mathrm{H}$ NMR techniques, the targeted identification of ten cyclohexadepsipeptides were achieved from the gorgonian coral-derived fungus Penicillium chrysogenum (TA01-16) (Fig. 41), including the new compounds $\mathbf{2 3 5}-\mathbf{2 3 7}$ (chrysogeamides A-C) and 240-244 (hrysogeamides D-H), as well as the known compounds 238 and 239 (scopularides A and B)
(Fig. 42) (Hou et al. 2019b). These cyclohexadepsipeptides contain the same $D$-Leu fragment, and $\mathbf{2 3 5}$ and $\mathbf{2 4 2}$ are reported for the first time featuring a 3-hydroxy-4-methylhexanoic acid (HMHA) moiety of cyclodepsipeptides. Interestingly, isotope labelling feeding experiments demonstrated that ${ }^{13} \mathrm{C}_{1}$ - $L$-Leu was transformed into ${ }^{13} \mathrm{C}_{1}$ - $D$-Leu moiety in 238 and 239 indicating that $D$-Leu in these molecules may be epimerized from $L$-Leu by a leucine racemase. Compounds $\mathbf{2 3 5 - 2 3 9}$ displayed selective activity in promoting angiogenesis toward the $T g$ (Flkl: EGFP) transgenic zebra fish $D$. rerio embryo (Hou et al. 2019b). 
<smiles>CCCCCC[C@H]1OC(=O)c2c(O)c(CC=C(C)C)cc(O)c2[C@H]1OC</smiles>

(+)-eurotiumide A (223)

(-)-eurotiumide A (224)

(+)-eurotiumide B (225)<smiles>CCCCC[C@H]1OC(=O)c2c(O)c(CC=C(C)C)cc(O)c2[C@H]1OC</smiles>

(-)-eurotiumide B (226)<smiles>C/C=C/CC[C@H]1OC(=O)c2c(O)c(CC=C(C)C)cc(O)c2[C@H]1OC</smiles>

(+)-eurotiumide D (229)<smiles>CCC/C=C/C1OC(=O)c2c(O)c(CC=C(C)C)cc(O)c2[C@H]1OC</smiles>

(-)-eurotiumide E (232)<smiles>C/C=C/CC[C@H]1OC(=O)c2c(O)c(CC=C(C)C)cc(O)c2[C@H]1OC</smiles>

(+)-eurotiumide C (227)<smiles>C/C=C/CC[C@H]1OC(=O)c2c(O)c(CC=C(C)C)cc(O)c2[C@H]1OC</smiles>

(-)-eurotiumide $\mathrm{D}(\mathbf{2 3 0})$<smiles>CCCCC[C@H]1O[C@H](OC)c2c3c(cc(O)c2[C@@H]1OC)C=CC(C)(C)O3</smiles>

( \pm )-eurotiumide $F(233)$<smiles>C/C=C/CC[C@@H]1OC(=O)c2c(O)c(CC=C(C)C)cc(O)c2[C@H]1OC</smiles>

(-)-eurotiumide C (228)<smiles>CCC/C=C/[C@H]1OC(=O)c2c(O)c(CC=C(C)C)cc(O)c2[C@H]1OC</smiles>

(+)-eurotiumide E (231)<smiles>CCCCC[C@H]1O[C@H](OC)c2c3c(cc(O)c2[C@@H]1OC)C=CC(C)(C)O3</smiles>

( \pm )-eurotiumide G (234)

Fig. 40 Dihydroisocoumarin enantiomers from the gorgonian-derived fungus Eurotium sp. XS-200900E6

Applying molecular networking techniques, three new cycloheptapeptides, 245-247 (asperversiamides A-C) (Fig. 43), were isolated from the coral-derived fungus Aspergillus versicolor (CHNSCLM-0063) (Hou et al. 2019c). Their complete structures including configuration were confirmed by total synthesis. These compounds showed strong inhibitory activity against Mycobacterium marinum (MICs $=23.4,81.2,87.5 \mu \mathrm{mol} / \mathrm{L}$, respectively), demonstrating valuable application potential in treating M. marinum infection (Hou et al. 2019c).

Until now, the molecular networking approach has only been applied to mine the undiscovered cycloheptapeptides from marine-derived fungus in our research on MNPs. Undoubtedly, the molecular networking strategy based on LC-MS/MS and relevant data libraries should be considered an effectively targeted isolation technique to speed the discovery of new cryptic secondary metabolites. It is worth applying the molecular networking approach to exploit novel molecules from the pools of MNPs in extracts from marine organisms.

From the gorgonian-derived fungus Aspergillus sp. XS20090B 15, four lumazine peptides, 248 (penilumamide) and 249-251 (penilumamides B-D), together with a cyclic pentapeptide, 252 (asperpeptide A) (Fig. 44), were isolated (Chen et al. 2014c). Penilumamide B (249) was obtained from a feeding culture experiment with $L$-methionine.
Compounds 249-251 are rare lumazine peptides, of which 248 and 250 are formed from 249 by oxidation of the $L$-methionine residue. The cyclic pentapeptide (252) exhibited antibacterial activity against $B$. cereus and $S$. epidermidis $(\mathrm{MICs}=12.5 \mu \mathrm{mol} / \mathrm{L})($ Chen et al. $2014 \mathrm{c})$.

\section{Phenyl ether derivatives}

An increasing number of marine-derived phenyl ether derivatives with novel structures have been reported with diverse biological activities, including antimicrobial, antitumor, cardiotonic, antidiabetic, antiinflammatory and antiviral activities. For instance, 4-carbglyceryl-3,30-dihydroxy5,50-dimethyldiphenyl ether, a diphenyl ether isolated from the deep-sea-derived fungus Aspergillus versicolor SCSIO 41502 showed potent antifouling activity (Huang et al. 2017). A series of antibacterial polybrominated diphenyl ethers were isolated from the Indonesian sponge Lamellodysidea herbacea (Hanif et al. 2007). In our studies, 35 phenyl ether derivatives were obtained in all, among them 9 compounds were first reported, showing antibacterial, antifouling and cytotoxic activities (Chen et al. 2013b; Shi et al. 2017). To clarify the structure-activity relationships, chemical synthesis was performed (Chen et al. 2013b). 


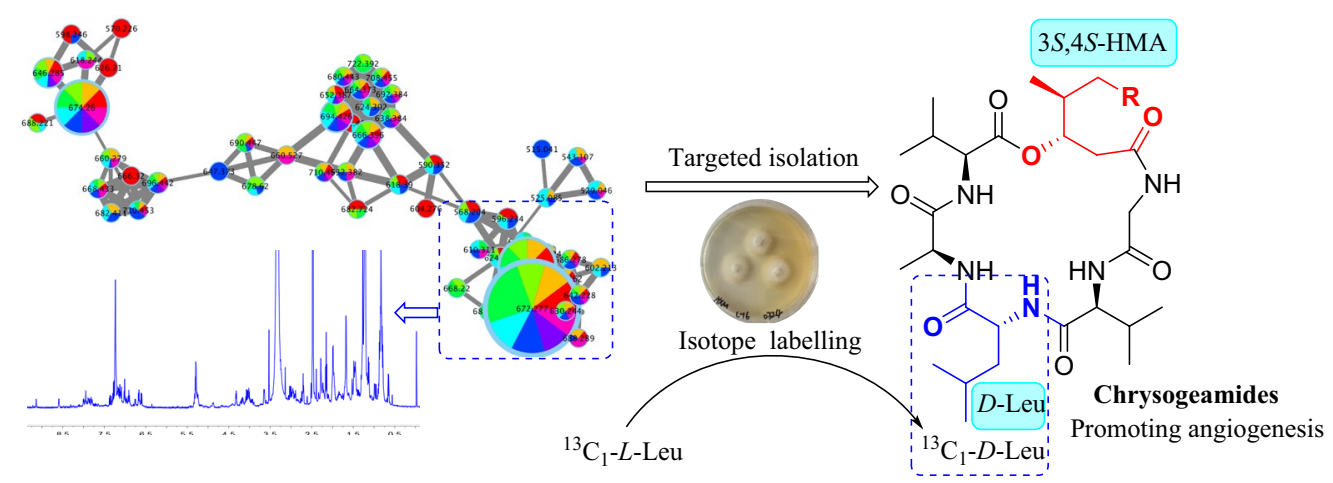

Fig. 41 The conceptual framework of LC-MS/MS-dependent molecular networking to target the isolation of new compounds

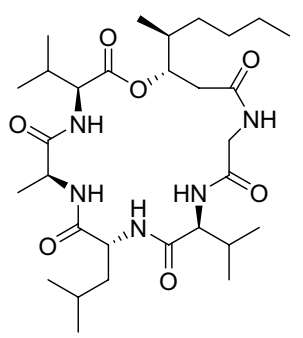

chrysogeamide A (235)<smiles>CCCCC(C)[C@H](CC(=O)NCC(=O)N[C@@H](CC(C)C)C(=O)N[C@@H](CC(C)C)C(=O)N[C@@H](C)C(=O)N[C@@H](Cc1ccccc1)C(=O)N[C@@H](C)CCCC)Cc1ccccc1</smiles>

chrysogeamide E (239)

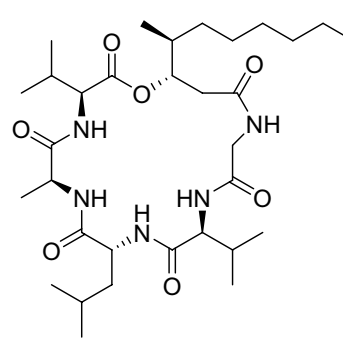

chrysogeamide B (236)<smiles>CCCC[C@H](C)[C@H](CC(=O)NCC(=O)N1CCC[C@H]1C(=O)N[C@@H](CC(C)C)C(=O)NC(C)C(=O)N[C@@H](Cc1ccccc1)C(=O)O)OC</smiles>

chrysogeamide F (240)<smiles>CCCCCCC(C)[C@H](CC(=O)NCC(=O)N[C@@H](CC(C)C)C(=O)N[C@@H](CC(C)C)C(=O)N[C@@H](C)C(=O)N[C@@H](Cc1ccccc1)C(=O)N[C@@H](C)CCCC)OC</smiles>

chrysogeamide C (237)<smiles>CCCCCC[C@H](C)[C@H](CC(=O)NCC(=O)N1CCCC1C(=O)N[C@@H](CC(C)C)C(=O)NC(C)C(=O)N[C@@H](Cc1ccccc1)C(=O)NC)OC</smiles>

chrysogeamide G (241)<smiles>CCC(C)[C@H](CC(=O)NCC(=O)N[C@@H](CC(C)C)C(=O)N[C@@H](CC(C)C)C(=O)N[C@@H](Cc1ccccc1)C(=O)N[C@@H](C)C(=O)N[C@@H](CC(C)C)C(C)C)Cc1ccccc1</smiles>

chrysogeamide D (238)<smiles>CCC(C)CC(=O)NCC(=O)NC(C(=O)O[C@H](CC(C)C)C(=O)N[C@@H](CC(C)C)C(=O)N[C@@H](C)C(=O)N[C@@H](C)C(C)C)C(C)C</smiles>

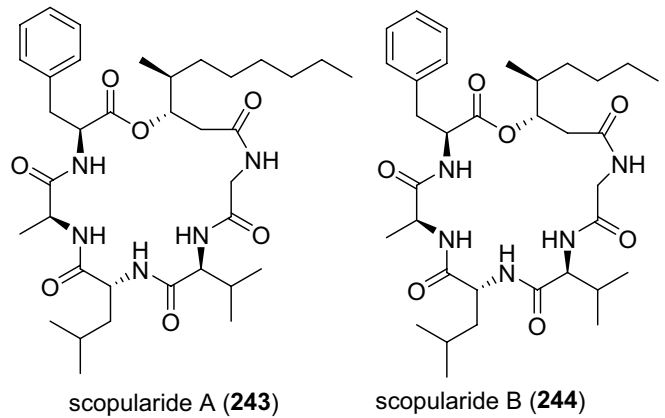

Fig. 42 Cyclohexadepsipeptides from the gorgonian coral-derived fungus $P$. chrysogenum (TA01-16)

Six phenyl ether derivatives were obtained from Aspergillus sp. XS-20090066 isolated from the gorgonian Dichotella gemmacea, including two new phenyl ether derivatives, 253 and 257 (cordyols D and E), together with 4 known analogue compounds 254 (3,3'-O-dimethyl-violaceol-I), 255 (cordyol C), 256 (4-methoxycarbonyl-diorcinol), and 258 (diorcinol) (Fig. 45) (Chen et al. 2013b). Among them, 256 displayed significant antibacterial activity against $S$. 
Fig. 43 Cycloheptapeptides from the coral-derived fungus A. versicolor (CHNSCLM-0063)

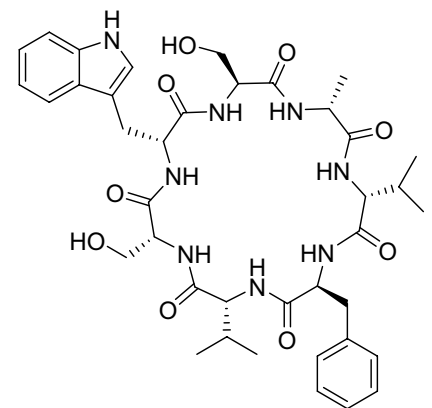

asperversiamide $\mathrm{A}(\mathbf{2 4 5})$<smiles>CC(C)C(=O)N[C@@H](C)C(=O)N[C@@H](C)C(=O)N[C@@H](Cc1c[nH]c2ccccc12)C(=O)N[C@@H](Cc1ccccc1)C(=O)N[C@@H](CO)C(=O)N[C@@H]1NC(=O)[C@H](C(C)C)NC(=O)[C@@H](C(C)C)NC1=O</smiles>

asperversiamide B (246)

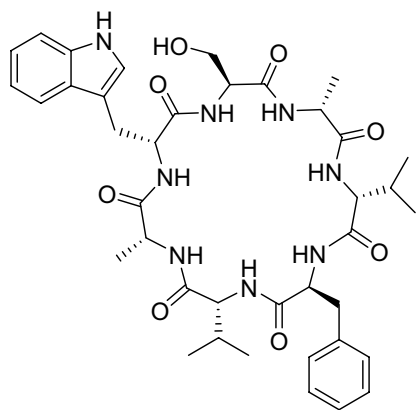

asperversiamide C (247) epidermidis $(\mathrm{MIC}=2.71 \mu \mathrm{mol} / \mathrm{L})$. A series of derivatives (256a, 258a-258g) were designed and synthesized for the study of structure-activity relationships, suggesting that one free hydroxy plays a critical role for antibacterial activity. In particular, 258g exhibited the strongest antibacterial activity toward $S$. epidermidis $(\mathrm{MIC}=0.556 \mu \mathrm{mol} / \mathrm{L})$. These results revealed that the ester functionality or bromination could increase antibacterial activity, but the simultaneous presence of both the ester and brominated benzene ring caused a loss of activity (Chen et al. 2013b).

Two new diphenyl glycosides, 259-260 (phomaethers A-B), one new diphenyl ether derivative, 261 (phomaether $\mathrm{C}$ ), and one known diphenyl ether analog, 262 (2,3'-dihydroxy-4-methoxy-5',6-dimethyl diphenyl ether) (Fig. 46), were isolated from a gorgonian-derived fungus
Phoma sp. (TA07-1) (Shi et al. 2017). It was the first report to find diphenyl glycoside derivatives from coral-derived fungi. Compounds 259, 261, 262 exhibited potent antibacterial activities, indicating that they might be developed as promising antibacterial agents. Specifically, 259 showed remarkable antibacterial activity against $S$. albus, $S$. aureus, E. coli and V. parahaemolyticus $(\mathrm{MICs}=0.312$ to $0.625 \mu \mathrm{mol} / \mathrm{L}$; minimum bactericidal concentrations $(\mathrm{MBCs})=0.625$ to $2.50 \mu \mathrm{mol} / \mathrm{L})$. Compound 261 displayed significant antibacterial activity to $S$. albus, $S$. aureus and E. $\operatorname{coli}(\mathrm{MICs}=0.312$ to $1.25 \mu \mathrm{mol} / \mathrm{L} ; \mathrm{MBCs}=0.625$ to $5.00 \mu \mathrm{mol} / \mathrm{L})$. Notably, 262 demonstrated strong antibacterial activity against all the tested pathogenic bacteria (MICs and $\mathrm{MBCs}=0.156$ to $5.00 \mu \mathrm{mol} / \mathrm{L})$ (Shi et al. 2017).
Fig. 44 Lumazine peptides and cyclic pentapeptide from gorgonian-derived fungus Aspergillus sp. XS-20090B15

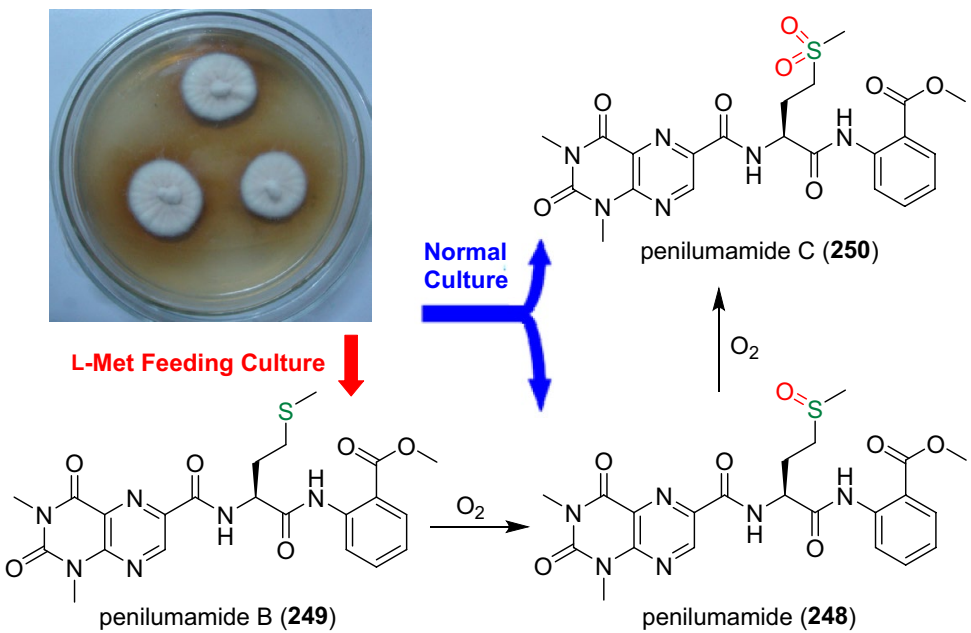<smiles>C[C@H](NC(=O)c1cnc2c(n1)c(=O)n(C)c(=O)n2C)C(=O)Nc1ccccc1C(N)=O</smiles>

penilumamide D (251)

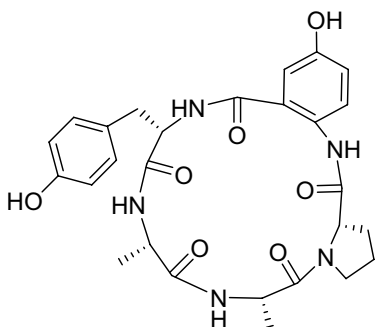

asperpeptide A (252) 
Besides those indicated above, other phenyl ether derivatives, obtained from various fungi including Penicillium pinophilum, Talaromyces sp. and Aspergillus elegans isolated from gorgonian and soft coral, also exhibited antilarval settlement activity against $B$. amphitrite and cytotoxic activity towards the tested human cell lines (Chen et al. 2015; Zhao et al. 2015a). For instance, diphenyl ether derivatives 263 (talaromycin C), 264 (purpactin $C^{\prime}$ ), 265 (pencillide) and 266 (purpactin A) (Fig. 47) displayed strong anti-larval settlement activity against $B$. amphitrite $\left(\mathrm{EC}_{50}=2.2-4.8 \mu \mathrm{g} / \mathrm{mL}\right.$ ). Compound 267 (tenellic acid A methyl ester) (Fig. 47) showed remarkable cytotoxicity towards human cell lines HepG2, Hep3B, MCF-7/ADR, PC-3, and HCT-116 $\left(\mathrm{IC}_{50}=4.3-9.8 \mu \mathrm{mol} / \mathrm{L}\right)($ Chen et al. 2015).

In summary, our research on the discovery of MNPs resulted in the discovery of diverse structures from soft corals, gorgonians, sponges, tunicates, anemones and their symbiotic microorganisms collected from the South China Sea. Based on our findings, the statistical analysis of 709 MNPs including 307 new compounds demonstrated that the proportions of terpenoids, alkaloids, steroids, and macrocyclic lactones are larger than that of other structures (Fig. 48). It is also revealed that macrocyclic lactones, azaphilones and phenylpropanoids are the most potential classes for the discovery of novel MNPs (Fig. 48). In terms of biological activities, more than half of the obtained MNPs displayed tested biological activities, with the most frequent findings as antibacterial, antifouling, and cytotoxic activities (Fig. 49). Interestingly, many of the active MNPs exhibited multiple bioactivities, for example, MNPs with antiviral activities usually displayed cytotoxic activities, while MNPs with antibacterial activities commonly showed cytotoxic and antifouling activities. It is worth noting that more than $40 \mathrm{com}$ pounds were found to exhibit potent activities even stronger than the positive controls. Particularly, the macrocyclic<smiles>COc1cc(C)c(Oc2cc(C)cc(Oc3cc(C)cc(O)c3O)c2O)c(Oc2cc(C)cc(Oc3c(OC)cc(C)cc3Oc3cc(C)cc(OC)c3OC)c2O)c1O</smiles><smiles>COC(=O)c1c(C)c(Br)c(Oc2c(Br)c(C)c(Br)c(O)c2Br)c(Br)c1O</smiles>

256a<smiles>CC(=O)Oc1cc(C)cc(Oc2cc(C)cc(OC(C)=O)c2)c1</smiles>

258b<smiles>CCCOc1cc(C)cc(Oc2cc(C)cc(O)c2)c1</smiles><smiles>COc1cc(C)cc(Oc2cc(C)cc(O)c2)c1</smiles>

cordyol E (257)<smiles>CC(=O)Oc1cc(C)cc(Oc2cc(C)cc(O)c2)c1</smiles><smiles>CCCCCOc1cc(C)cc(Oc2cc(C)cc(O)c2)c1</smiles><smiles>CCCCCOc1cc(C)cc(Oc2cc(C)cc(OCCCCC)c2)c1</smiles>

258c 258d<smiles>Cc1cc(O)cc(Oc2cc(C)cc(OC(=O)c3ccccc3)c2)c1</smiles><smiles>Cc1c(Br)c(O)c(Br)c(Oc2c(Br)c(Br)c(O)c(Br)c2Br)c1Br</smiles>

Fig. 45 Phenyl ether derivatives from the gorgonian-derived fungus Aspergillus sp. XS-20090066<smiles>COc1cc(C)c(Oc2cc(C)cc(O)c2)c(OCOC(O)C(O)CO)c1</smiles>

phomaether A (259)<smiles>COc1cc(C)c(Oc2cc(C)cc(OC3OC(CO)C(O)C3O)c2)c(O)c1</smiles>

phomaether B (260)<smiles>COc1cc(O)c(Oc2cc(C)cc(O)c2)c(C)c1OC</smiles>

phomaether C (261)<smiles>COc1cc(C)c(Oc2cc(C)cc(O)c2)c(O)c1</smiles>

2,3'-dihydroxy-4-methoxy $-5^{\prime}, 6$-dimethyl diphenyl ether (262)

Fig. 46 Diphenyl glycosides and diphenyl ether derivatives from the gorgonian-derived fungus Phoma sp. (TA07-1) 
lactone cochliomycin A (1), the azaphilone sclerotioramine derivative (101), and the alkaloid aniduquinolone A (104) may be candidates for efficient antifouling agents, and the macrocyclic lactone bastimolide $\mathrm{A}$ (36), the anthraquinones nigrosporin B (41), and the steroid echrebsteroid C (165) might be promising lead compounds for further development of antimalarial, antibiotic, and antiviral drugs.

The South China Sea possesses rich and unique species resources, with more than $95 \%$ of the invertebrates mainly existing in the coral reefs in this sea area in China (Zhang et al. 2006). Not surprisingly, many investigations on marine invertebrates and their symbiotic microorganisms target this "biodiversity hotspot" as the center of sample collection. It should be mentioned that the diverse abundance of MNPs derived from marine invertebrates and their symbiotic microorganisms from the South China Sea were discovered and studied. In recent decades, research on the diversity of MNPs discovered from the South China Sea by other Chinese researchers has been well underway. These groundbreaking research efforts contributed to the discovery of a great number of novel and promising bioactive molecules usually from such sources as sponges (Gui et al. 2019; Jiao et al. 2019; Wang et al. 2015b), corals (Li et al. 2019b; Wu et al. 2019), bryozoans (Yu et al. 2015), and associated microorganisms (Cheng et al. 2016; Nong et al. 2016). Regarding the abundance of MNPs with diverse structures and a wealth of biological activities, we believe that only the proverbial "tip of the iceberg" has been explored from the South China Sea, and the resulting novel active metabolites with potential pharmacology applications are worthy of further exploration.
Fig. 47 Diphenyl ether derivatives from the gorgonianderived fungus Talaromyces sp.
Fig. 48 The distribution of MNPs obtained from marine invertebrates and their symbiotic microorganisms
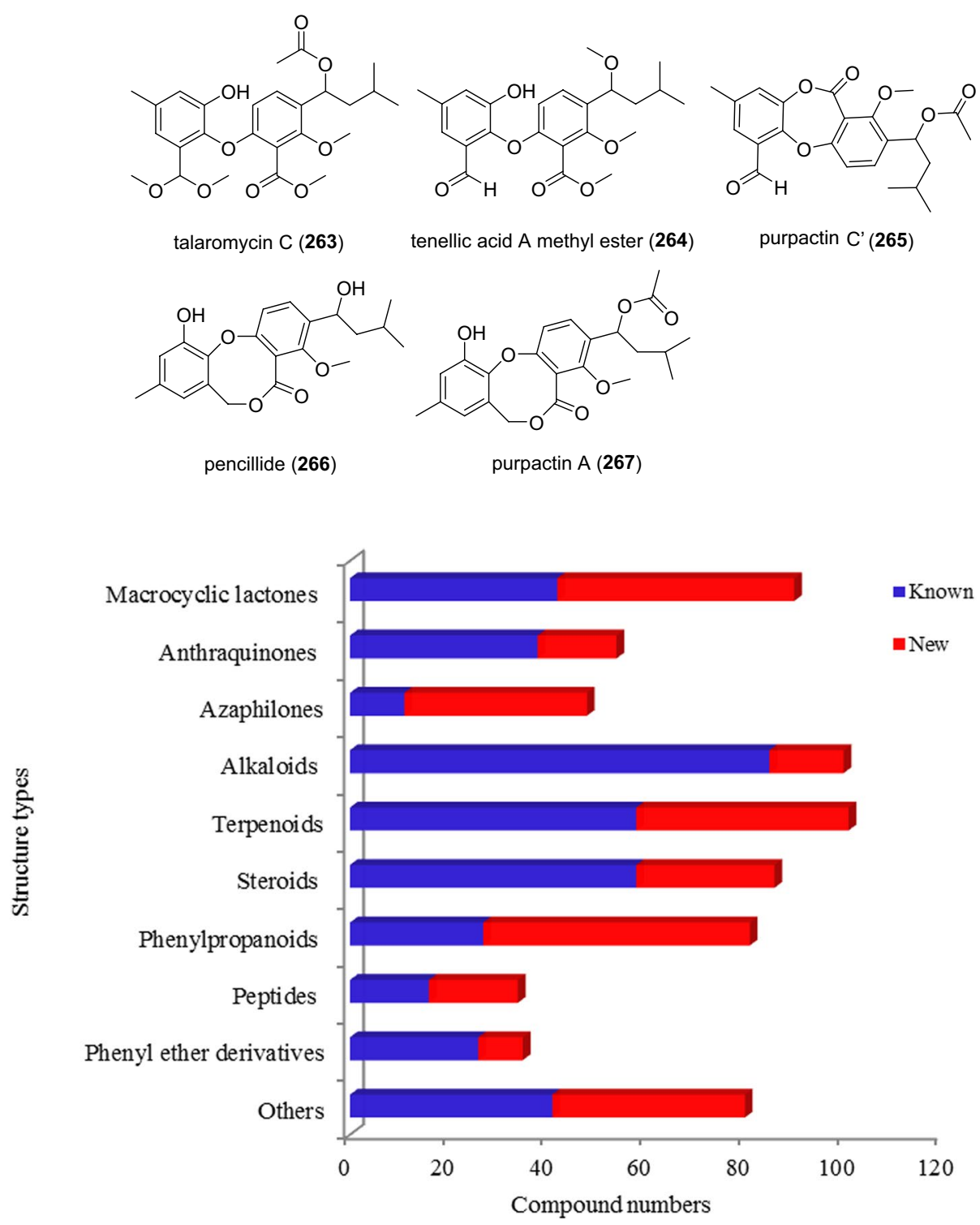
Fig. 49 The frequency of MNPs based on diverse biological activities

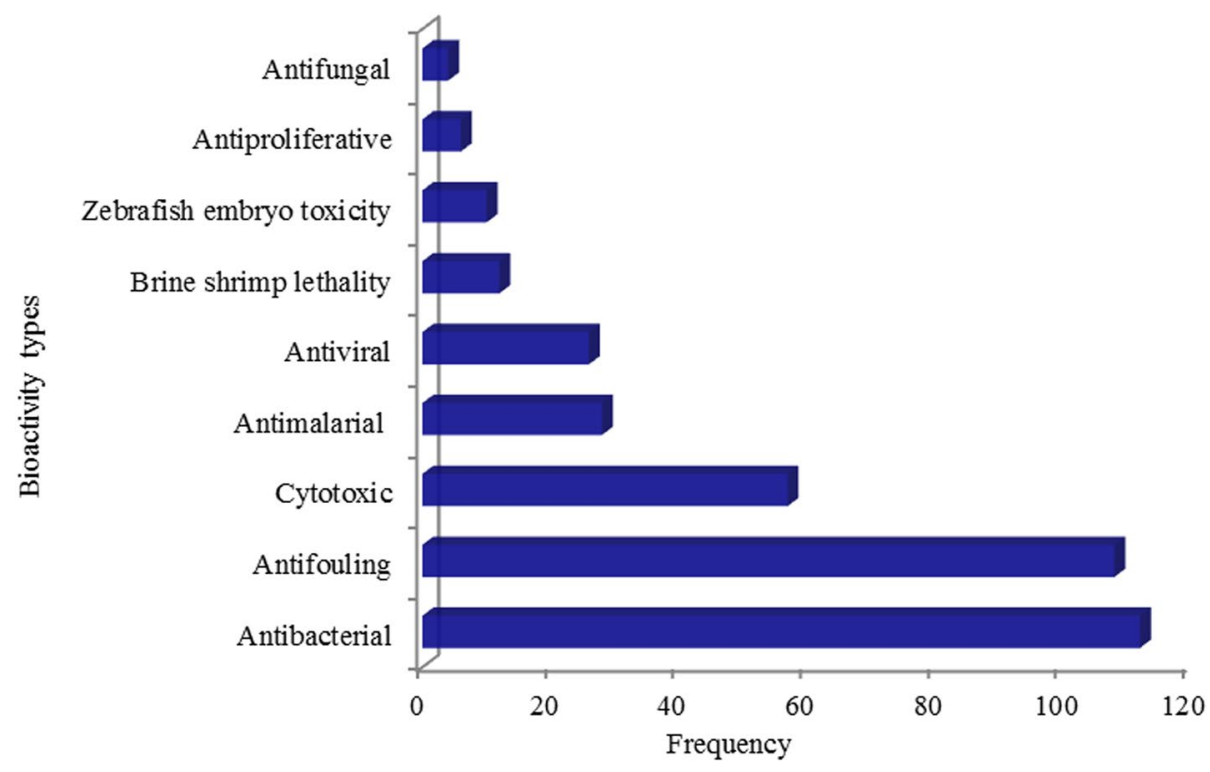

\section{Conclusion}

In this review, we exemplified nine types of structurally unique MNPs including macrocyclic lactones, anthraquinones, azaphilones, alkaloids, terpenoids, steroids, phenylpropanoids, peptides, and phenyl ether derivatives obtained from marine invertebrates and their symbiotic microorganisms. Our research provides several typical representative MNPs from marine invertebrates, especially sponges, soft corals, gorgonian corals, and their symbiotic microorganisms (mainly fungi). These MNPs display various potent bioactivities involved in not only chemoecological effects such as antifouling, ichthyootoxic, and brine shrimp lethal activities but also pharmaceutical activities including antibacterial, antiviral, fungicidal, cytotoxic, and antimalarial activities. Our studies demonstrate that MNPs derived from marine invertebrates and their symbiotic microorganisms in the South China Sea are a prolific resource for the discovery of bioactive MNPs. It could be expected that the symbiotic microorganisms associated with marine invertebrates have great potential as a significant source of structurally interesting molecules. It should be noted that the application of multiple discovery strategies and methods could effectively promote the exploitation of novel MNPs with diverse structures. In our study, different methodological approaches, including single culture, OSMAC, chemical epigenetic manipulation, co-culture, structural modification and chemical synthesis, have been applied in the search for new MNPs. Among them, co-culture, structural modification and chemical synthesis were found to be effective approaches to obtain potential bioactive MNPs. Particularly, LC-MS/ MS-dependent molecular networking has been applied as a promising approach to dereplicate complex natural product mixtures, contributing to the targeted isolation of a series of new compounds. It is anticipated that future genetic techniques and bioinformatics tools, especially metagenomic approaches, genome mining, and heterologue biosynthesis, could accelerate the exploration and accessibility of remaining undiscovered MNPs with novel structures and promising bioactivities from marine microorganisms.

Acknowledgements This work is supported by the National Natural Science Foundation of China (nos. 41830535; 81673350; U1706210), the Fundamental Research Funds for the Central Universities of China (no. 201962002), and the Taishan Scholars Program, China.

Author contributions LL and YYZ collected the data and wrote the paper; CLS and CYW designed, directed and revised the manuscript.

\section{Compliance with ethical standards}

Conflict of interest The authors declare that they have no conflict of interest.

Animal and human rights statement This article does not contain any studies with human participants or animals performed by any of the authors.

Open Access This article is distributed under the terms of the Creative Commons Attribution 4.0 International License (http://creativeco mmons.org/licenses/by/4.0/), which permits unrestricted use, distribution, and reproduction in any medium, provided you give appropriate credit to the original author(s) and the source, provide a link to the Creative Commons license, and indicate if changes were made.

\section{References}

Abe T, Nakamura S, Yanada R, Choshi T, Hibino S, Ishikura M (2013) One-pot construction of 3,3'-bisindolylmethanes through Bartoli indole synthesis. Org Lett 15:3622-3625 
Altmann KH (2017) Drugs from the oceans: marine natural products as leads for drug discovery. Chimia 71:646-652

Bandini M, Eichholzer A (2009) Catalytic functionalization of indoles in a new dimension. Angew Chem Int Edit 48:9608-9644

Bao J, He F, Yu JH, Zhai HJ, Cheng ZQ, Jiang CS, Zhang YY, Zhang Y, Zhang XY, Chen GY, Zhang H (2018) New chromones from a marine-derived fungus, Arthrinium sp., and their biological activity. Molecules 23:1982

Blunt JW, Carroll AR, Copp BR, Davis RA, Keyzers RA, Prinsep MR (2018) Marine natural products. Nat Prod Rep 35:8-53

Cao F, Zhou J, Xu KX, Zhang MQ, Wang CY (2013) New cembranoid diterpene from the South China Sea soft coral Sarcophyton sp. Nat Prod Commun 8:1675-1678

Cao F, Shao CL, Chen M, Zhang MQ, Xu KX, Meng H, Wang CY (2014) Antiviral C-25 epimers of 26-acetoxy steroids from the South China Sea gorgonian Echinogorgia rebekka. J Nat Prod 77:1488-1493

Cao F, Wu ZH, Shao CL, Pang S, Liang XY, de Voogd NJ, Wang CY (2015) Cytotoxic scalarane sesterterpenoids from the South China Sea sponge Carteriospongia foliascens. Org Biomol Chem 46:4016-4024

Cao F, Shao CL, Liu YF, Zhu HJ, Wang CY (2017) Cytotoxic serrulatane-type diterpenoids from the gorgonian Euplexaura sp. and their absolute configurations by vibrational circular dichroism. Sci Rep 7:12548

Cao F, Meng ZH, Mu X, Yue YF, Zhu HJ (2019) Absolute configuration of bioactive azaphilones from the marine-derived fungus Pleosporales sp. CF09-1. J Nat Prod 82:386-392

Carroll AR, Copp BR, Davis RA, Keyzers RA, Prinsep MR (2019) Marine natural products. Nat Prod Rep 36:122-173

Che YS, Gloer JB, Koster B, Malloch D (2002) Decipinin A and decipienolides A and B: new bioactive metabolites from the coprophilous fungus Podospora decipiens. J Nat Prod 65:916-919

Chen CH, Xiao WW, Jiang XB, Wang JW, Mao ZG, Lei N, Fan X, Song BB, Liao CX, Wang HJ, She ZG, Zhu YH (2013a) A novel marine drug, SZ-685C, induces apoptosis of MMQ pituitary tumor cells by downregulating miR-200c. Curr Med Chem 20:2145-2154

Chen M, Shao CL, Fu XM, Xu RF, Zheng JJ, Zhao DL, She ZG, Wang CY (2013b) Bioactive indole alkaloids and phenyl ether derivatives from a marine-derived Aspergillus sp. fungus. J Nat Prod 76:547-553

Chen M, Shao CL, Fu XM, Kong CJ, She ZG, Wang CY (2014a) Lumazine peptides penilumamides B-D and the cyclic pentapeptide asperpeptide A from a gorgonian-derived Aspergillus sp. fungus. J Nat Prod 77:1601-1606

Chen M, Shao CL, Meng H, She ZG, Wang CY (2014b) Anti-respiratory syncytial virus prenylated dihydroquinolone derivatives from the gorgonian-derived fungus Aspergillus sp. XS20090B15. J Nat Prod 77:2720-2724

Chen M, Shao CL, Wang KL, Xu Y, She ZG, Wang CY (2014c) Dihydroisocoumarin derivatives with antifouling activities from a gorgonian-derived Eurotium sp. fungus. Tetrahedron 70:9132-9138

Chen M, Han L, Shao CL, She ZG, Wang CY (2015) Bioactive diphenyl ether derivatives from a gorgonian-derived fungus Talaromyces $\mathrm{sp}$. Chem Biodivers 12:443-450

Chen M, Wu XD, Zhao Q, Wang CY (2016) Topsensterols A-C, cytotoxic polyhydroxylated sterol derivatives from a marine sponge Topsentia sp. Mar Drugs 14:146-153

Cheng ZB, Lou LL, Liu D, Li XD, Proksch P, Yin S, Lin WH (2016) Versiquinazolines A-K, fumiquinazoline-type alkaloids from the gorgonian-derived fungus Aspergillus versicolor LZD-14-1. J Nat Prod 79:2941-2952

Deshmukh SK, Prakash V, Ranjan N (2017) Marine fungi: a source of potential anticancer compounds. Front Microbiol 8:2536
Ebrahim W, Aly AH, Mándi A, Totzke F, Kubbutat MHG, Wray V, Lin WH, Dai HF, Proksch P, Kurtán T, Debbab A (2012) Decalactone derivatives from Corynespora cassiicola, an endophytic fungus of the mangrove plant Laguncularia racemosa. Eur J Org Chem 18:3476-3484

Ebrahim W, Aly AH, Wray V, Proksch P, Debbab A (2013) Unusual octalactones from Corynespora cassiicola, an endophyte of Laguncularia racemosa. Tetrahedron Lett 54:6611-6614

Faulkner DJ (1984) Marine natural products: metabolites of marine algae and herbivorous marine mollusks. Nat Prod Rep 12:51-80

Fu LP, Shi QQ, Shi Y, Jiang B, Tu SJ (2013) Three-component domino reactions for regioselective formation of bis-indole derivatives. ACS Comb Sci 15:135-140

Gui YH, Jiao WH, Zhou M, Zhang Y, Zeng DQ, Zhu HR, Liu KC, Sun F, Chen HF, Lin HW (2019) Septosones A-C, in vivo antiinflammatory meroterpenoids with rearranged carbon skeletons from the marine sponge Dysidea septosa. Org Lett 21:767-770

Hanif N, Tanaka J, Setiawan A, Trianto A, de Voogd NJ, Murni A, Tanaka C, Higa T (2007) Polybrominated diphenyl ethers from the Indonesian sponge Lamellodysidea herbacea. J Nat Prod $70: 432-435$

Hibino S, Choshi T (2001) Simple indole alkaloids and those with a nonrearranged monoterpenoid unit (1999). Nat Prod Rep 18:66-87

Hirata Y, Uemura D (1986) Halichondrins-antitumor polyether macrolides from a marine sponge. Pure Appl Chem 58:701-710

Hong SW, Singh AJ, Patel V, Russell ER, Field JJ, Miller JH, Northcote PT (2018) Peloruside E (22-Norpeloruside A), a pelorusane macrolide from the New Zealand marine sponge Mycale hentscheli, retains microtubule-stabilizing properties. J Nat Prod 81:2125-2128

Hou XM, Xu RF, Gu YC, Wang CY, Shao CL (2015) Biological and chemical diversity of coral-derived microorganisms. Curr Med Chem 22:3707-3762

Hou XM, Hai Y, Gu YC, Wang CY, Shao CL (2019a) Chemical and bioactive marine natural products of coral-derived microorganisms (2015-2017). Curr Med Chem 26:1. https://doi. org/10.2174/0929867326666190626153819

Hou XM, Li YY, Shi YW, Fang YW, Chao R, Gu YC, Wang CY, Shao CL (2019b) Integrating molecular networking and ${ }^{1} \mathrm{H}$ NMR to target the isolation of chrysogeamides from a library of marine-derived Penicillium fungi. J Org Chem $84: 1228-1237$

Hou XM, Liang TM, Guo ZY, Wang CY, Shao CL (2019c) Discovery, absolute assignments, and total synthesis of asperversiamides A-C and their potent activity against Mycobacterium marinum. Chem Commun 55:1104-1107

Huang CY, Chang CW, Tseng YJ, Lee J, Sung PJ, Su JH, Hwang TL, Dai CF, Wang HC, Sheu JH (2016) Bioactive steroids from the formosan soft coral Umbellulifera petasites. Mar Drugs $14: 180-190$

Huang ZH, Nong XH, Ren Z, Wang J, Zhang XY, Qi SH (2017) AntiHSV-1, antioxidant and antifouling phenolic compounds from the deep-sea-derived fungus Aspergillus versicolor SCSIO 41502. Bioorg Med Chem Lett 27:787-791

Humphrey GR, Kuethe JT (2006) Practical methodologies for the synthesis of indoles. Chem Rev 106:2875-2911

Isaka M, Suyarnsestakorn C, Tanticharoen M (2002) Aigialomycins A-E, new resorcylic macrolides from the marine mangrove fungus Aigialus parvus. J Org Chem 67:1561-1566

Isaka M, Yangchum A, Intamas S, Kocharin K, Jones EBG, Kongsaeree P, Prabpai S (2009) Aigialomycins and related polyketide metabolites from the mangrove fungus Aigialus parvus $\mathrm{BCC}$ 5311. Tetrahedron 65:4396-4403

Jia YL, Wei MY, Chen HY, Guan FF, Wang CY, Shao CL (2015) (+)and (-)-Pestaloxazine A, a pair of antiviral enantiomeric alkaloid 
dimers with a symmetric spiro[oxazinane-piperazinedione] skeleton from Pestalotiopsis sp. Org Lett 17:4216-4219

Jiao WH, Li J, Zhang MM, Cui J, Gui YH, Zhang Y, Li JY, Liu KC, Lin HW (2019) Frondoplysins A and B, unprecedented terpene-alkaloid bioconjugates from Dysidea frondosa. Org Lett 21:6190-6193

Jiménez C (2018) Marine natural products in medicinal chemistry. ACS Med Chem Lett 9:959-961

Kamauchi H, Noji M, Kinoshita K, Takanami T, Koyama K (2018) Coumarins with an unprecedented tetracyclic skeleton and coumarin dimers from chemically engineered extracts of a marinederived fungus. Tetrahedron 74:2846-2856

Kochanowska-Karamyan AJ, Hamann MT (2010) Marine indole alkaloids: potential new drug leads for the control of depression and anxiety. Chem Rev 110:4489-4497

Leal MC, Hilário A, Munro MHG, Blunt JW, Calado R (2016) Natural products discovery needs improved taxonomic and geographic information. Nat Prod Rep 33:747-750

Li L, Sheng L, Wang CY, Zhou YB, Huang H, Li XB, Li J, Mollo E, Gavagnin M, Guo YW (2011) Diterpenes from the Hainan soft coral Lobophytum cristatum Tixier-Durivault. J Nat Prod 74:2089-2094

Li D, Xu Y, Shao CL, Yang RY, Zheng CJ, Chen YY, Fu XM, Qian PY, She ZG, de Voogd NJ, Wang CY (2012a) Antibacterial bisabolane-type sesquiterpenoids from the sponge-derived fungus Aspergillus sp. Mar Drugs 10:234-241

Li R, Shao CL, Qi X, Li XB, Li J, Sun LL, Wang CY (2012b) Polyoxygenated sterols from the South China Sea soft coral Sinularia sp. Mar Drugs 10:1422-1432

Li HL, Li XM, Li X, Wang CY, Liu H, Kassack MU, Meng LH, Wang BG (2016) Antioxidant hydroanthraquinones from the marine algal-derived endophytic fungus Talaromyces islandicus EN-501. J Nat Prod 80:162-168

Li G, Li H, Zhang Q, Yang M, Gu YC, Liang LF, Tang W, Guo YW (2019a) Rare cembranoids from Chinese Soft Coral Sarcophyton ehrenbergi: structural and stereochemical studies. J Org Chem 84:5091-5098

Li J, Hu YY, Hao XM, Tan JL, Li F, Qiao XR, Chen SZ, Xiao CL, Chen MH, Peng Z, Gan M (2019b) Raistrickindole A, an antiHCV oxazinoindole alkaloid from Penicillium raistrickii IMB17034. J Org Chem 82:1391-1395

Liu QA, Shao CL, Gu YC, Blum M, Gan LS, Wang KL, Chen M, Wang CY (2014) Antifouling and fungicidal resorcylic acid lactones from the sea anemone-derived fungus Cochliobolus lunatus. J Agr Food Chem 62:3183-3191

Liu ZM, Qiu P, Liu HJ, Li J, Shao CL, Yan T, Cao WH, She ZG (2019) Identification of anti-inflammatory polyketides from the coral-derived fungus Penicillium sclerotiorin: in vitro approaches and molecular-modeling. Bioorg Chem 88:102973. https://doi. org/10.1016/j.bioorg.2019.102973

Lounasmaa M, Tolvanen A (2000) Simple indole alkaloids and those with a nonrearranged monoterpenoid unit (July 1997 to December 1998). Nat Prod Rep 17:175-191

Ma SM, Yu SC (2005) Sc(OTf) ${ }_{3}$-catalyzed indolylation of 1,2-allenic ketones: controlled highly selective synthesis of $\beta$-indolyl- $\alpha$, $\beta$-unsaturetated $(E)$-enones and $\beta, \beta$-bisindolyl ketones. Org Lett 7:5063-5065

Martins A, Vieira H, Gaspar H, Santos S (2014) Marketed marine natural products in the pharmaceutical and cosmeceutical industries: tips for success. Mar Drugs 12:1066-1101

Mayer AMS, Glaser KB, Cuevas C, Jacobs RS, Kem W, Little RD, McIntosh JM, Newman DJ, Potts BC, Shuster DE (2010) The odyssey of marine pharmaceuticals: a current pipeline perspective. Trends Pharmacol Sci 31:255-265

Newman DJ (2019) The "utility" of highly toxic marine-sourced compounds. Mar drugs 17:324. https://doi.org/10.3390/md17060324
Newman DJ, Cragg GM (2016a) Drugs and drug candidates from marine sources: an assessment of the current "state of play". Planta Med 82:775-789

Newman DJ, Cragg GM (2016b) Natural products as sources of new drugs from 1981 to 2014. J Nat Prod 79:629-661

Nong XH, Zhang XY, Xu XY, Wang J, Qi SH (2016) Nahuoic acids B-E, polyhydroxy polyketides from the marine-derived Streptomyces sp. SCSGAA 0027. J Nat Prod 79:141-148

Pereira RB, Valentao P, Pereira DM, Valentão P, Kornienko A, Pereira DM, Andrade PB, Gomes NGM (2019) Marine-derived anticancer agents: clinical benefits, innovative mechanisms, and new targets. Mar Drugs 17:329. https://doi.org/10.3390/md17060329

Perez-Victoria I, Oves-Costales D, Lacret R, Martín J, Sánchez-Hidalgo M, Díaz C, Cautain B, Vicente F, Genilloud O, Reyes F (2019) Structure elucidation and biosynthetic gene cluster analysis of caniferolides A-D, new bioactive 36-membered macrolides from the marine-derived Streptomyces caniferus CA-271066. Org Biomol Chem 17:2954-2971

Pettit GR, Ducki S, Herald DL, Doubek DL, Schmidt JM, Chapuis JC (2005) Antineoplastic agents 470. Absolute configuration of the marine sponge bromopyrrole agelastatin A. Oncol Res 15:11-20

Plaza A, Gustchina E, Baker HL, Kelly M, Bewley CA (2007) Mirabamides A-D, depsipeptides from the sponge Siliquariaspongia mirabilis that inhibit HIV-1 fusion. J Nat Prod 70:1753-1760

Qi Y, Ma S (2011) The medicinal potential of promising marine macrolides with anticancer activity. Chem Med Chem 6:399-409

Qi J, Shao CL, Li ZY, Gan LS, Fu XM, Bian WT, Zhao HY, Wang CY (2013) Isocoumarin derivatives and benzofurans from a spongederived Penicillium sp. fungus. J Nat Prod 76:571-579

Shao CL, Wu HX, Wang CY, Liu QA, Xu Y, Wei MY, Qian PY, Gu YC, Zheng CJ, She ZG, Lin YC (2011) Potent antifouling resorcylic acid lactones from the gorgonian-derived fungus Cochliobolus lunatus. J Nat Prod 74:629-633

Shao CL, Linington RG, Balunas MJ, Centeno A, Boudreau P, Zhang C, Engene N, Spadafora C, Mutka TS, Kyle DE, Gerwick L, Wang CY, Gerwick WH (2015a) Bastimolide A, a potent antimalarial polyhydroxy macrolide from the marine cyanobacterium Okeania hirsuta. J Org Chem 80:7849-7855

Shao CL, Xu RF, Wang CY, Qian PY, Wang KL, Wei MY (2015b) Potent antifouling marine dihydroquinolin-2(1H)-one-containing alkaloids from the gorgonian coral-derived fungus Scopulariopsis sp. Mar Biotechnol 17:408-415

Shao CL, Mou XF, Cao F, Spadafora C, Glukhov E, Gerwick L, Wang CY, Gerwick WH (2018) Bastimolide B, an antimalarial 24-membered marine macrolide possessing a tert-butyl group. J Nat Prod 81:211-215

Shi T, Qi J, Shao CL, Zhao DL, Hou XM, Wang CY (2017) Bioactive diphenyl ethers and isocoumarin derivatives from a gorgonianderived fungus Phoma sp. (TA07-1). Mar Drugs 15:146

Shier WT, Shier AC, Xie W, Mirocha CJ (2001) Structure-activity relationships for human estrogenic activity in zearalenone mycotoxins. Toxicon 39:1435-1438

Song YP, Fang ST, Miao FP, Yin XL, Ji NY (2018) Diterpenes and sesquiterpenes from the marine algicolous fungus Trichoderma harzianum X-5. J Nat Prod 81:2553-2559

Sun P, Meng LY, Tang H, Liu BS, Li L, Yi YH, Zhang W (2012) Sinularosides A and B, bioactive 9,11-secosteroidal glycosides from the South China Sea soft coral Sinularia humilis Ofwegen. J Nat Prod 75:1656-1659

Sun XP, Cao F, Shao CL, Chen M, Liu HJ, Zheng CJ, Wang CY (2015) Subergorgiaols A-L, 9,10-secosteroids from the South China Sea gorgonian Subergorgia rubra. Steroids 94:7-14

Suo R, Takada K, Kohtsuka H, Ise Y, Okada S, Matsunaga S (2018) Miuramides A and B, trisoxazole macrolides from Mycale sp. marine sponge that induce a protrusion phenotype in cultured mammalian cells. J Nat Prod 81:1108-1112 
Wang CY, Liu HY, Shao CL, Wang YN, Li L, Guan HS (2008) Chemical defensive substances of soft corals and gorgonians. Acta Ecol Sin 28:2320-2328

Wang CY, Chen AN, Shao CL, Li L, Xu Y, Qian PY (2011a) Chemical constituents of soft coral Sarcophyton infundibuliforme from the South China Sea. Biochem Syst Ecol 39:853-856

Wang H, Wang Y, Wang W, Fu P, Liu PP, Zhu WM (2011b) Anti-influenza virus polyketides from the acid-tolerant fungus Penicillium purpurogenum JS03-21. J Nat Prod 74:2014-2018

Wang KL, Xu Y, Lu L, Li YX, Han Z, Zhang J, Shao CL, Wang CY, Qian PY (2015a) Low-toxicity diindol-3-ylmethanes as potent antifouling compounds. Mar Biotechnol 17:624-632

Wang Q, Tang XL, Luo XC, de Voogd NJ, Li PL, Li GQ (2015b) (+)and (-)-spiroreticulatine, a pair of unusual spiro bisheterocyclic quinolone-imidazole alkaloids from the South China Sea sponge fascaplysinopsis reticulata. Org Lett 17:3458-3461

Wang KL, Zhang G, Sun J, Xu Y, Han Z, Liu LL, Shao CL, Liu QA, Wang CY, Qian PY (2016) Cochliomycin A inhibits the larval settlement of Amphibalanus amphitrite, by activating the NO/ cGMP pathway. Biofouling 32:35-44

Wang J, Mu FR, Jiao WH, Huang J, Hong LL, Yang F, Xu Y, Wang SP, Sun F, Lin HW (2017) Meroterpenoids with protein tyrosine phosphatase 1B inhibitory activity from a Hyrtios sp. marine sponge. J Nat Prod 80:2509-2514

Wang CY, Hao JD, Ning XY, Wu JS, Zhao DL, Kong CJ, Shao CL, Wang CY (2018) Penicilazaphilones D and E: two new azaphilones from a sponge-derived strain of the fungus Penicillium sclerotiorum. RSC Adv 8:4348-4353

Wei MY, Wang CF, Wang KL, Qian PY, Wang CY, Shao CL (2017) Preparation, structure, and potent antifouling activity of sclerotioramine derivatives. Mar Biotechnol 19:372-378

Wen H, Wang L, Xu L, Hao ZH, Shao CL, Wang CY, Xiao J (2015) Fluorinated alcohol-mediated $\mathrm{S}_{\mathrm{N}} 1$-type reaction of indolyl alcohols with diverse nucleophiles. Adv Synth Catal 357:4023-4030

Wu Q, Ye F, Li XL, Liang LF, Sun J, Sun H, Guo YW, Wang H (2019) Uncommon polyoxygenated sesquiterpenoids from South China Sea soft coral Lemnalia flava. J Org Chem 84:3083-3092

Xie GE, Zhu X, Li Q, Gu MH, He ZJ, Wu JH, Li J, Lin YC, Li MF, She ZG, Yuan J (2010) SZ-685C, a marine anthraquinone, is a potent inducer of apoptosis with anticancer activity by suppression of the Akt/FOXO pathway. Br J Pharmacol 159:689-697

Yamada T, Doi M, Shigeta H, Muroga Y, Hosoe S, Numata A, Tanaka R (2008) Absolute stereostructures of cytotoxic metabolites, chaetomugilins A-C, produced by a Chaetomium species separated from a marine fish. Tetrahedron Lett 49:4192-4195

Yamada T, Suzue M, Arai T, Kikuchi T, Tanaka R (2017) Trichodermanins C-E, new diterpenes with a fused 6-5-6-6 ring system produced by a marine sponge-derived fungus. Mar Drugs 15:169

Yang KL, Wei MY, Shao CL, Fu XM, Guo ZY, Xu RF, Zheng CJ, She ZG, Lin YC, Wang CY (2012) Antibacterial anthraquinone derivatives from a sea anemone-derived fungus Nigrospora sp. J Nat Prod 75:935-941

Yasukawa K, Itabashi T, Kawai KI, Takido M (2008) Inhibitory effects of falconensins on 12-O-tetradecanoylphorbol-13-acetateinduced inflammatory ear edema in mice. J Nat Med 62:384-386

Yu HF, Yu ZK (2009) Direct alkenylation of indoles with $\alpha$-oxo ketene dithioacetals: efficient synthesis of indole alkaloids meridianin derivatives. Angew Chem Int Edit 48:2929-2933

Yu HB, Yang F, Li YY, Gan JH, Jiao WH, Lin HW (2015) Cytotoxic bryostatin derivatives from the South China Sea bryozoan Bugula neritina. J Nat Prod 78:1169-1173
Yu ML, Guan FF, Cao F, Jia YL, Wang CY (2018) A new antiviral pregnane from a gorgonian-derived Cladosporium sp. fungus. Nat Prod Res 32:1260-1266

Yu ML, Li YX, Banakar SP, Liu L, Shao CL, Li ZY, Wang CY (2019) New metabolites from the co-culture of marine-derived actinomycete Streptomyces rochei MB037 and fungus Rhinocladiella similis 35. Front Microbiol 10:915. https://doi.org/10.3389/fmicb .2019 .00915

Zhan KX, Jiao WH, Yang F, Li J, Wang SP, Li YS, Han BN, Lin HW (2014) Reniochalistatins A-E, cyclic peptides from the marine sponge Reniochalina stalagmitis. J Nat Prod 77:2678-2684

Zhang W, Guo YW, Gu YC (2006) Secondary metabolites from the South China Sea invertebrates: chemistry and biological activity. Curr Med Chem 13:2041-2090

Zhang W, Shao CL, Chen M, Liu QA, Wang CY (2014) Brominated resorcylic acid lactones from the marine-derived fungus Cochliobolus lunatus, induced by histone deacetylase inhibitors. Tetrahedron Lett 55:4888-4891

Zhang XQ, Spadafora C, Pineda LM, Ng MG, Sun JH, Wang W, Wang CY, Gu YC, Shao CL (2017) Discovery, semisynthesis, antiparasitic and cytotoxic evaluation of 14-membered resorcylic acid lactones and their derivatives. Sci Rep 7:11822

Zhao HY, Shao CL, Li ZY, Han L, Cao F, Wang CY (2013) Bioactive pregnane steroids from a South China Sea gorgonian Carijoa $\mathrm{sp.}$ Molecules 18:3458-3466

Zhao DL, Shao CL, Gan LS, Wang M, Wang CY (2015a) Chromone derivatives from a sponge-derived strain of the fungus Corynespora cassiicola. J Nat Prod 7:286-293

Zhao DL, Shao CL, Zhang Q, Wang KL, Guan FF, Shi T, Wang CY (2015b) Azaphilone and diphenyl ether derivatives from a gorgonian-derived strain of the fungus Penicillium pinophilum. J Nat Prod 78:2310-2314

Zheng CJ, Shao CL, Guo ZY, Chen JF, Deng DS, Yang KL, Chen YY, Fu XM, She ZG, Lin YC, Wang CY (2012) Bioactive hydroanthraquinones and anthraquinone dimers from a soft coral-derived Alternaria sp. fungus. J Nat Prod 75:189-197

Zheng JJ, Shao CL, Chen M, Gan LS, Fang YC, Wang XH, Wang CY (2014) Ochracenoids A and B, guaiazulene-based analogues from gorgonian Anthogorgia ochracea collected from the South China Sea. Mar Drugs 12:1569-1579

Zhou YM, Shao CL, Wang CY, Huang H, Xu Y, Qian PY (2011) Chemical constituents of the gorgonian Dichotella fragilis (Ridleg) from the South China Sea. Nat Prod Commun 6:1239-1242

Zhou SL, Wang M, Zhao HG, Huang YH, Lin YY, Tan GH, Chen SL (2016) Penicilazaphilone C, a new antineoplastic and antibacterial azaphilone from the marine fungus Penicillium sclerotiorum. Arch Pharm Res 39:1621-1627

Zhu YP, Liu MC, Jia FC, Yuan JJ, Gao QH, Lian M, Wu AX (2002) Metal-free $\mathrm{sp}^{3} \mathrm{C}-\mathrm{H}$ bond dual-(Het)arylation: $\mathrm{I}_{2}$-promoted domino process to construct 2,2-bisindolyl-1-arylethanones. Org Lett $14: 3392-3395$

Zhu X, He ZJ, Wu JH, Yuan J, Wen WT, Hu YW, Jiang Y, Lin CJ, Zhang QH, Lin M, Zhang HN, Yang W, Chen H, Zhong LL, She ZG, Chen SP, Lin YC, Li MF (2012) A marine anthraquinone SZ-685C overrides adriamycin-resistance in breast cancer cells through suppressing Akt signaling. Mar Drugs 10:694-711 